Supporting Information

\title{
Mixed Methyl Aryloxy Rare-Earth Metal Complexes Stabilized by a Superbulky Tris(pyrazolyl)borato Ligand
}

Verena M. Birkelbach, Christoph Stuhl, Cäcilia Maichle-Mössmer, and Reiner Anwander* Institut für Anorganische Chemie, Eberhard Karls Universität Tübingen, Auf der Morgenstelle 18, 72076 Tübingen (Germany)

* to whom correspondence should be addressed: E-Mail reiner.anwander@uni-tuebingen.de 


\section{Table of contents}

\section{NMR spectroscopy}

${ }^{1} \mathrm{H} N M R,{ }^{13} \mathrm{C}\left\{{ }^{1} \mathrm{H}\right\}$ NMR, ${ }^{11} \mathrm{~B}\left\{{ }^{1} \mathrm{H}\right\}$ NMR spectra of complex 1-Lu

Pages S3-S4

${ }^{1} \mathrm{H} N M R,{ }^{13} \mathrm{C}\left\{{ }^{1} \mathrm{H}\right\}$ NMR, ${ }^{11} \mathrm{~B}\left\{{ }^{1} \mathrm{H}\right\}$ NMR spectra of complex 2-Lu

Pages S4-S5

${ }^{1} \mathrm{H}$ NMR, ${ }^{13} \mathrm{C}\left\{{ }^{1} \mathrm{H}\right\}$ NMR, ${ }^{11} \mathrm{~B}\left\{{ }^{1} \mathrm{H}\right\}$ NMR, ${ }^{19} \mathrm{~F}\left\{{ }^{1} \mathrm{H}\right\}$ NMR spectra of complex 3-Lu

Pages S6-S7

${ }^{1} \mathrm{H} N M R,{ }^{13} \mathrm{C}\left\{{ }^{1} \mathrm{H}\right\}$ NMR, ${ }^{11} \mathrm{~B}\left\{{ }^{1} \mathrm{H}\right\}$ NMR, ${ }^{1} \mathrm{H}^{89} \mathrm{Y}$ HSQC NMR spectra of complex 4-Y

Pages S8-S10

${ }^{1} \mathrm{H} N M R,{ }^{13} \mathrm{C}\left\{{ }^{1} \mathrm{H}\right\}$ NMR, ${ }^{11} \mathrm{~B}\left\{{ }^{1} \mathrm{H}\right\}$ NMR spectra of complex 4-Lu

Pages S10-S11

${ }^{1} \mathrm{H} N M R,{ }^{13} \mathrm{C}\left\{{ }^{1} \mathrm{H}\right\}$ NMR, ${ }^{11} \mathrm{~B}\left\{{ }^{1} \mathrm{H}\right\}$ NMR, ${ }^{1} \mathrm{H}^{89} \mathrm{Y}$ HSQC NMR spectra of complex 5-Y

Pages S12-S13

${ }^{1} \mathrm{H} N M R,{ }^{13} \mathrm{C}\left\{{ }^{1} \mathrm{H}\right\}$ NMR, ${ }^{11} \mathrm{~B}\left\{{ }^{1} \mathrm{H}\right\}$ NMR spectra of complex 5-Lu

Pages S14-S15

${ }^{1} \mathrm{H} N M R,{ }^{13} \mathrm{C}\left\{{ }^{1} \mathrm{H}\right\}$ NMR, ${ }^{11} \mathrm{~B}\left\{{ }^{1} \mathrm{H}\right\}$ NMR, ${ }^{1} \mathrm{H}^{89} \mathrm{Y}$ HSQC NMR spectra of complex 6-Y

Pages S15-S17

${ }^{1} \mathrm{H} N M R,{ }^{13} \mathrm{C}\left\{{ }^{1} \mathrm{H}\right\}$ NMR, ${ }^{11} \mathrm{~B}\left\{{ }^{1} \mathrm{H}\right\}$ NMR spectra of complex 6-Lu

Pages S17-S18

${ }^{1} \mathrm{H}$ NMR, ${ }^{13} \mathrm{C}\left\{{ }^{1} \mathrm{H}\right\}$ NMR, ${ }^{11} \mathrm{~B}\left\{{ }^{1} \mathrm{H}\right\}$ NMR, ${ }^{19} \mathrm{~F}\left\{{ }^{1} \mathrm{H}\right\}$ NMR, ${ }^{1} \mathrm{H}-{ }^{89} \mathrm{Y}$ HSQC NMR spectra of Pages S19-S21 complex 7-Y

${ }^{1} \mathrm{H}$ NMR, ${ }^{13} \mathrm{C}\left\{{ }^{1} \mathrm{H}\right\}$ NMR, ${ }^{11} \mathrm{~B}\left\{{ }^{1} \mathrm{H}\right\}$ NMR, ${ }^{19} \mathrm{~F}\left\{{ }^{1} \mathrm{H}\right\}$ NMR spectra of complex 7-Lu

Pages S21-S23

${ }^{1} \mathrm{H} N M R,{ }^{13} \mathrm{C}\left\{{ }^{1} \mathrm{H}\right\}$ NMR, ${ }^{11} \mathrm{~B}\left\{{ }^{1} \mathrm{H}\right\}$ NMR,${ }^{1} \mathrm{H}_{-}{ }^{89} \mathrm{Y} H S Q C$ NMR spectra of complex 8-Y

Pages S24-S25

${ }^{1} \mathrm{H} N M R,{ }^{13} \mathrm{C}\left\{{ }^{1} \mathrm{H}\right\}$ NMR, ${ }^{11} \mathrm{~B}\left\{{ }^{1} \mathrm{H}\right\}$ NMR spectra of complex 8-Lu

Pages S26-S26

\section{X-ray structure analyses}

ORTEP representation of the molecular structure for compounds 1-Lu, 2-Lu, 3-Lu, 5-Lu, 6-Y, and 7-Lu

\section{$\underline{\text { Cone angle analyses }}$}




\section{NMR spectroscopy}

The solvent residual peaks are marked with an asterisk $\left(^{*}\right)$.

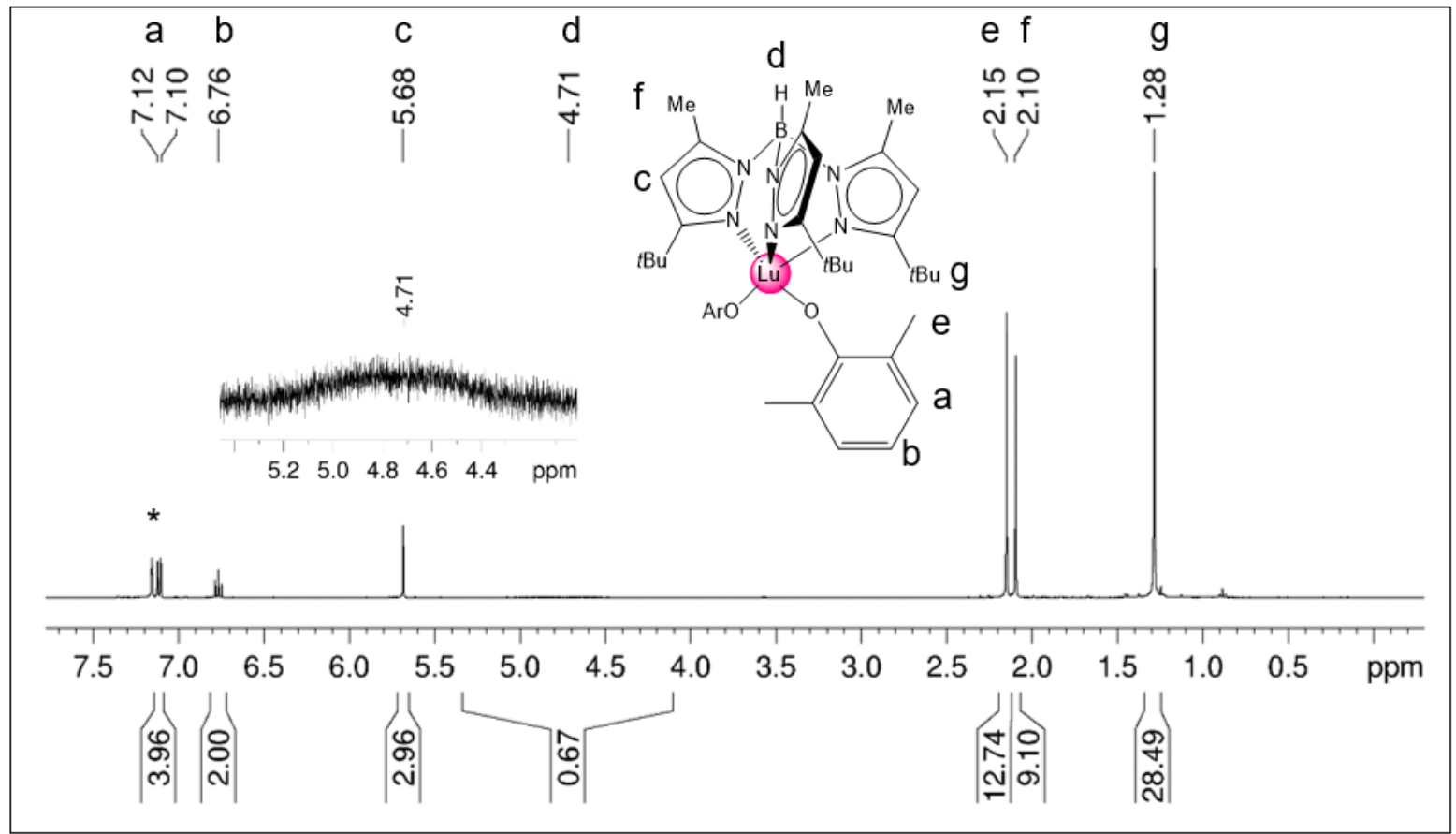

Figure S1. ${ }^{1} \mathrm{H}$ NMR spectrum $\left(250 \mathrm{MHz},\left[\mathrm{D}_{6}\right]\right.$ benzene) of complex $\mathrm{Tp}^{\mathrm{tBu}, \mathrm{Me}} \mathrm{Lu}\left(\mathrm{OC}_{6} \mathrm{H}_{3} \mathrm{Me}_{2}-2,6\right)_{2}$ (1-Lu) at $26{ }^{\circ} \mathrm{C}$.

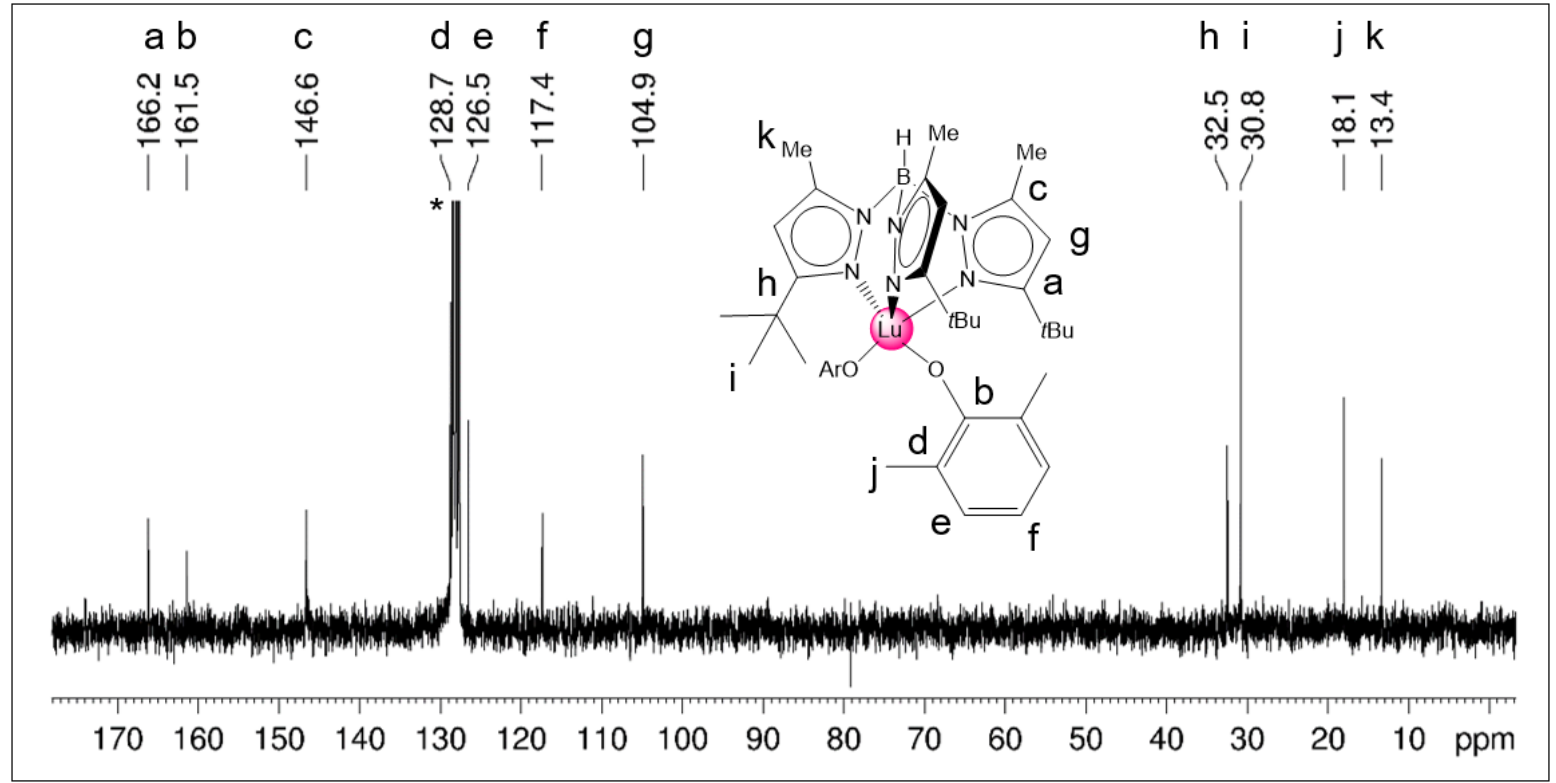

Figure S2. ${ }^{13} \mathrm{C}\left\{{ }^{1} \mathrm{H}\right\}$ NMR spectrum (63 MHz, $\left[\mathrm{D}_{6}\right]$ benzene) of complex $\mathrm{Tp}{ }^{\mathrm{tBu}, \mathrm{Me}} \mathrm{Lu}\left(\mathrm{OC}_{6} \mathrm{H}_{3} \mathrm{Me}_{2}-2,6\right)_{2}($ (1Lu) at $26^{\circ} \mathrm{C}$. 


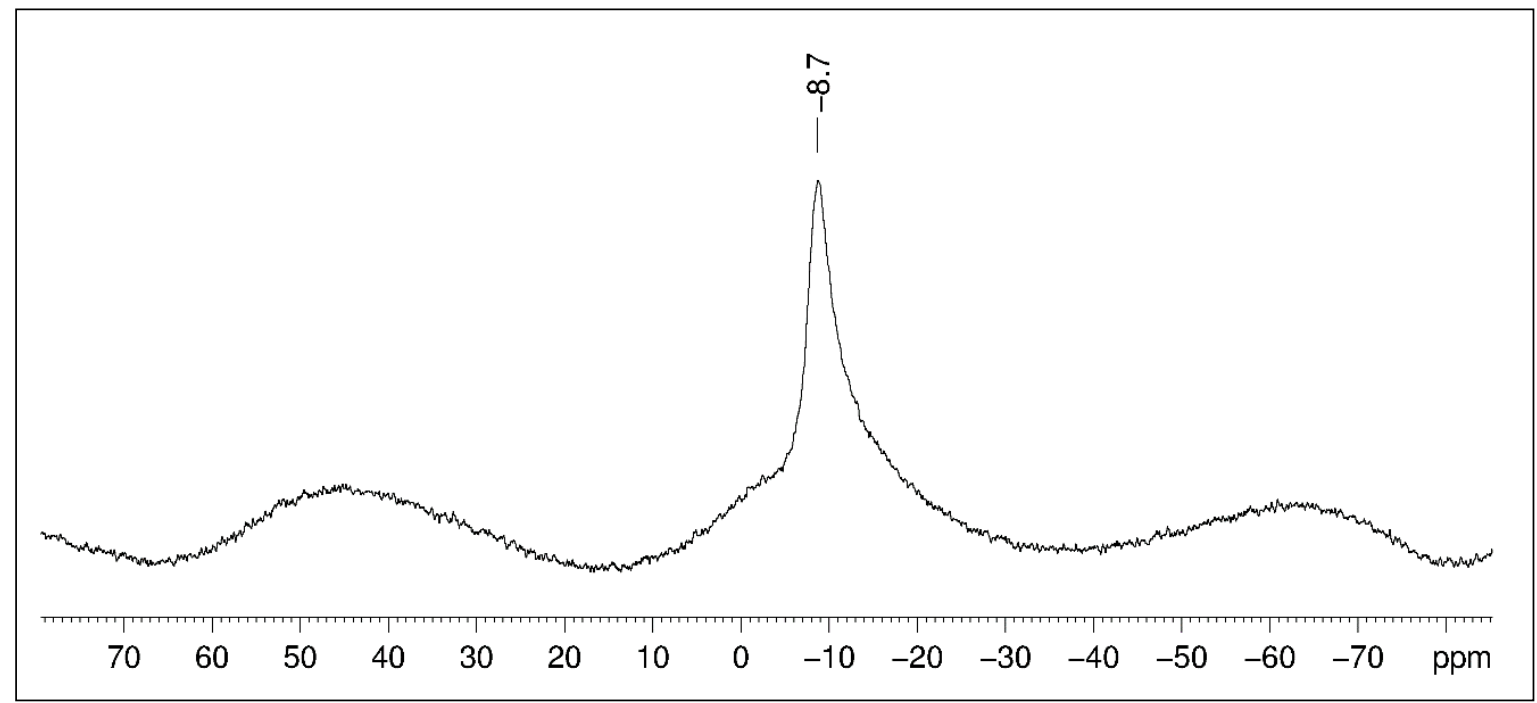

Figure S3. ${ }^{11} \mathrm{~B}\left\{{ }^{1} \mathrm{H}\right\} \mathrm{NMR}$ spectrum (80 MHz, [D 6 ]benzene) of complex $\mathrm{Tp}^{\mathrm{tBu}, \mathrm{Me}} \mathrm{Lu}\left(\mathrm{OC}_{6} \mathrm{H}_{3} \mathrm{Me}_{2}-2,6\right)_{2}$ (1Lu) at $26^{\circ} \mathrm{C}$.

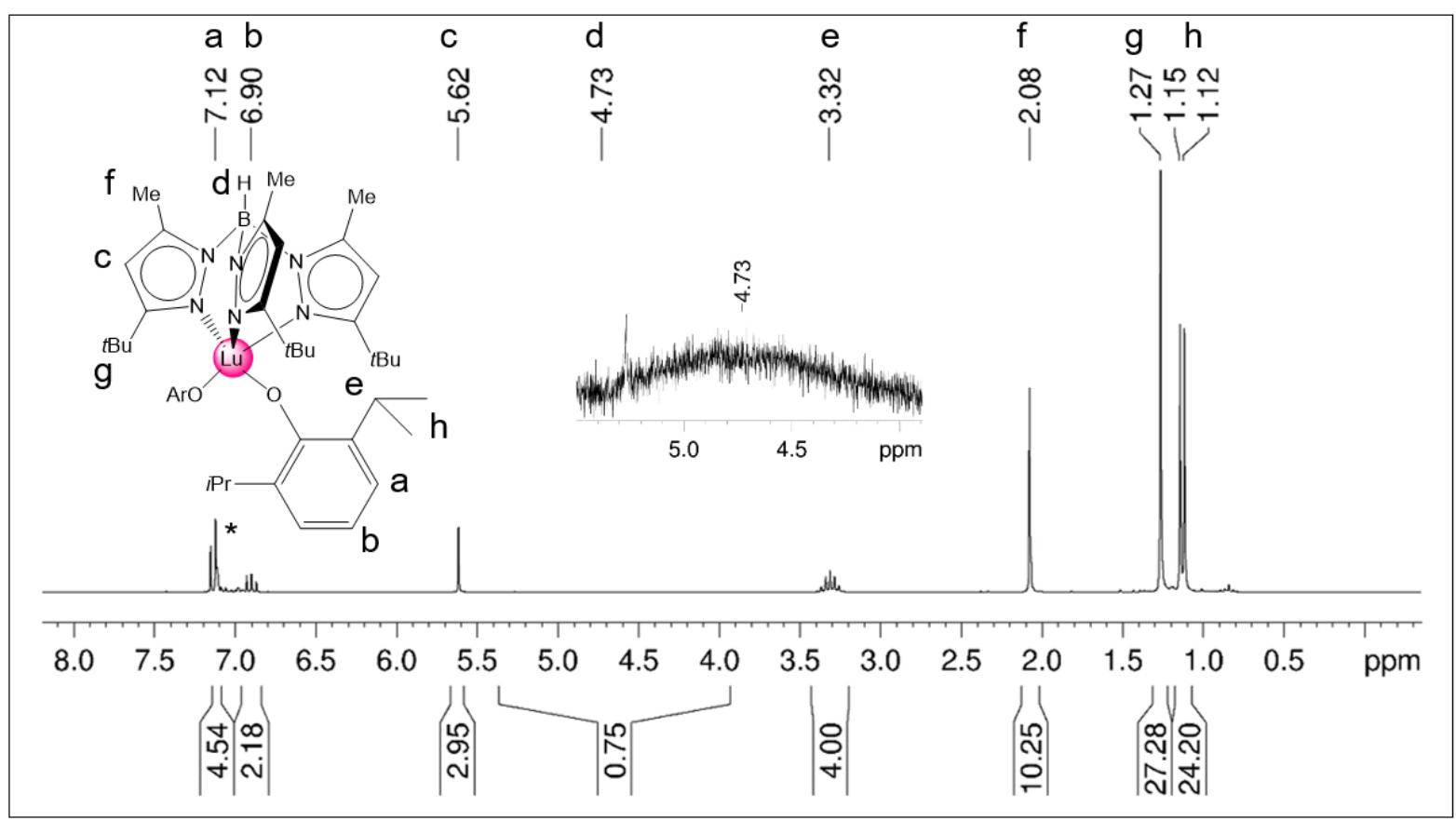

Figure S4. ${ }^{1} \mathrm{H}$ NMR spectrum (250 MHz, [D $\left.\mathrm{D}_{6}\right]$ benzene) of complex $\mathrm{Tp}^{\mathrm{tBu}, \mathrm{Me}} \mathrm{Lu}\left(\mathrm{OC}_{6} \mathrm{H}_{3} \mathrm{Pr}_{2}-2,6\right)_{2}(2-\mathrm{Lu})$ at $26^{\circ} \mathrm{C}$. 


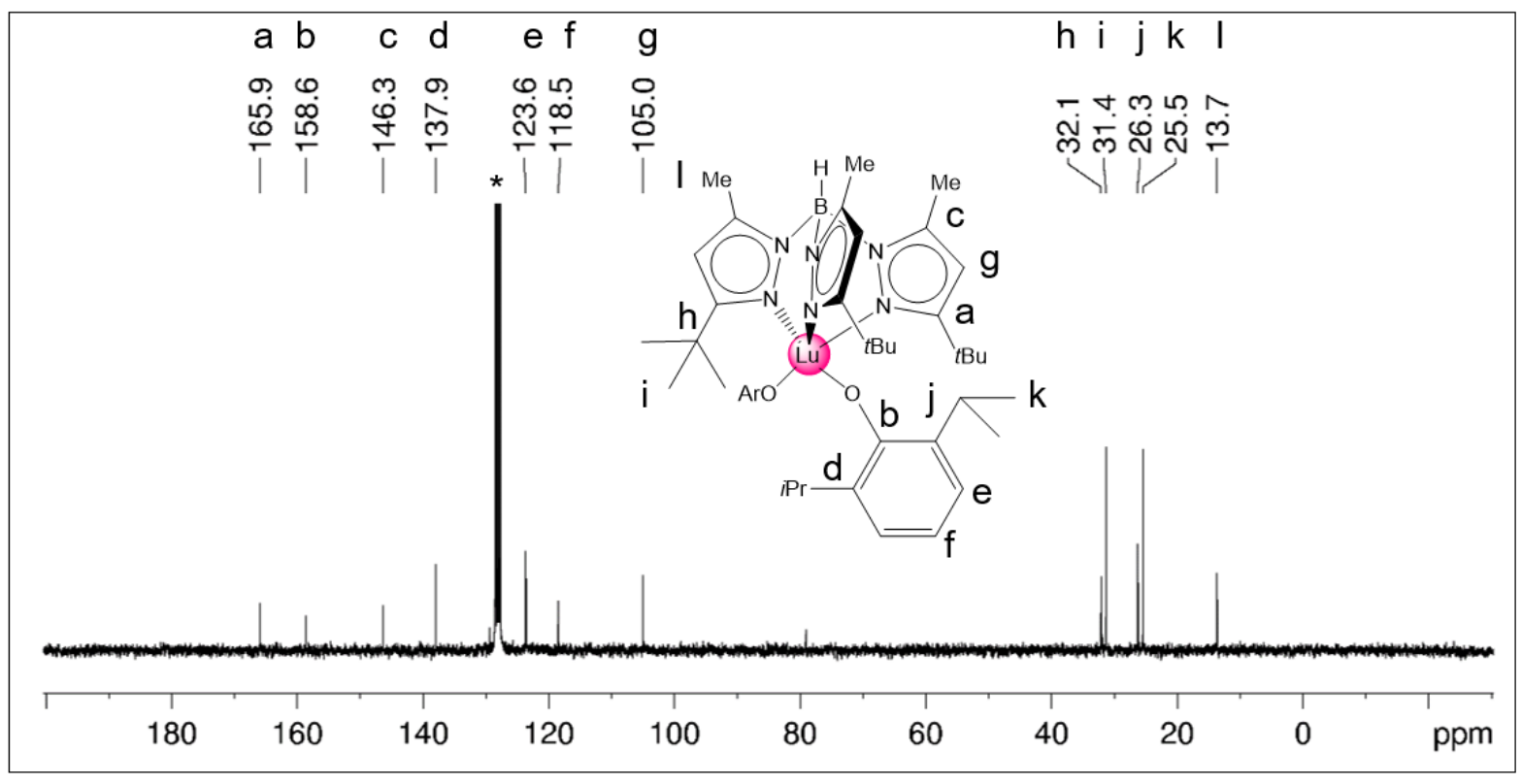

Figure S5. ${ }^{13} \mathrm{C}\left\{{ }^{1} \mathrm{H}\right\}$ NMR spectrum (63 MHz, $\left[\mathrm{D}_{6}\right]$ benzene) of complex $\mathrm{Tp}{ }^{\mathrm{tBu}, \mathrm{Me}} \mathrm{Lu}\left(\mathrm{OC}_{6} \mathrm{H}_{3} / \mathrm{Pr}_{2}-2,6\right)_{2}(2-$ Lu) at $26^{\circ} \mathrm{C}$.

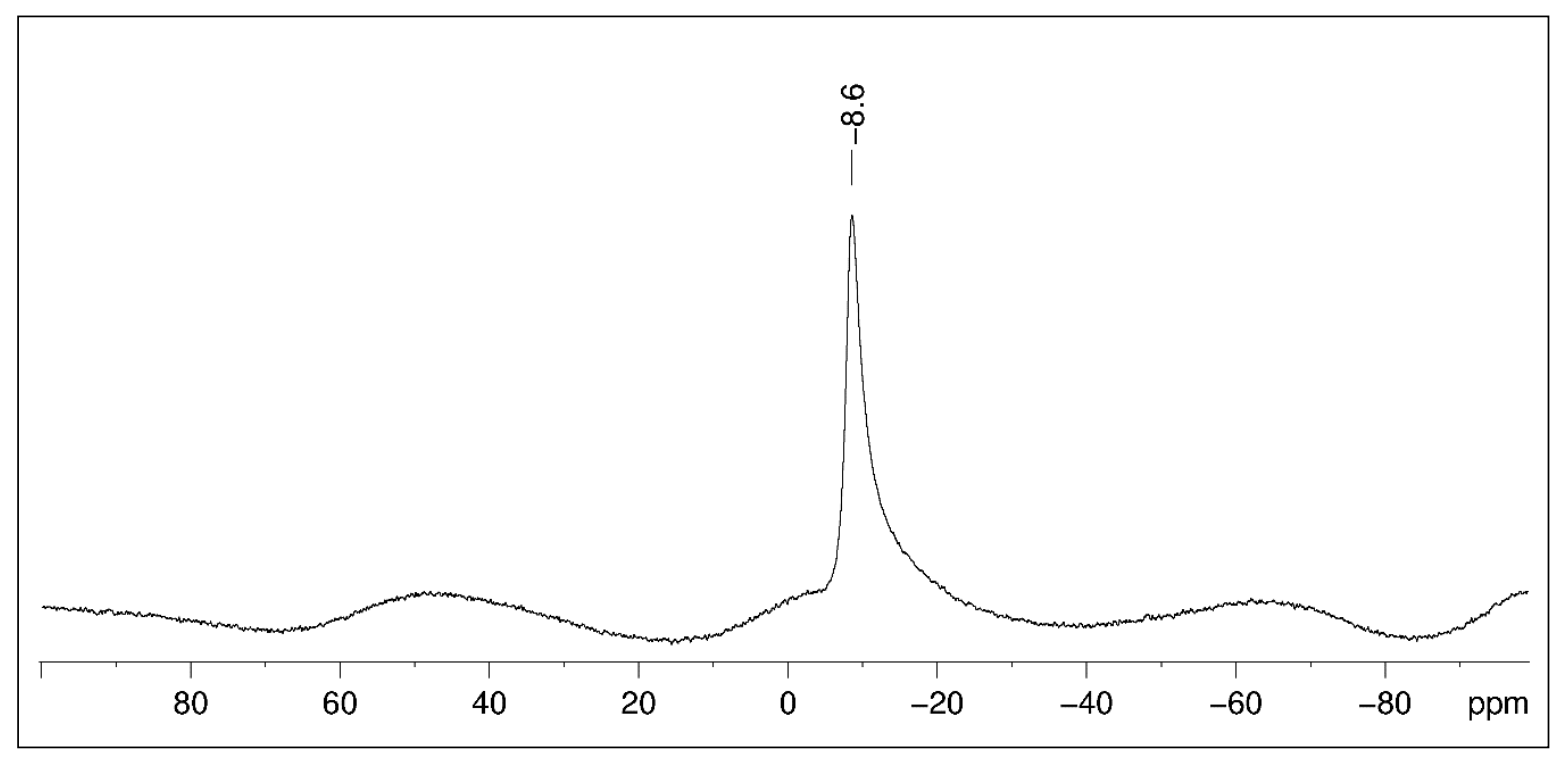

Figure S6. ${ }^{11} \mathrm{~B}\left\{{ }^{1} \mathrm{H}\right\} \mathrm{NMR}$ spectrum (80 MHz, [D6] benzene) of complex $\mathrm{Tp}^{\mathrm{tBu}, \mathrm{Me}} \mathrm{Lu}\left(\mathrm{OC}_{6} \mathrm{H}_{3} / \mathrm{Pr}_{2}-2,6\right)_{2}(2-$ Lu) at $26^{\circ} \mathrm{C}$. 


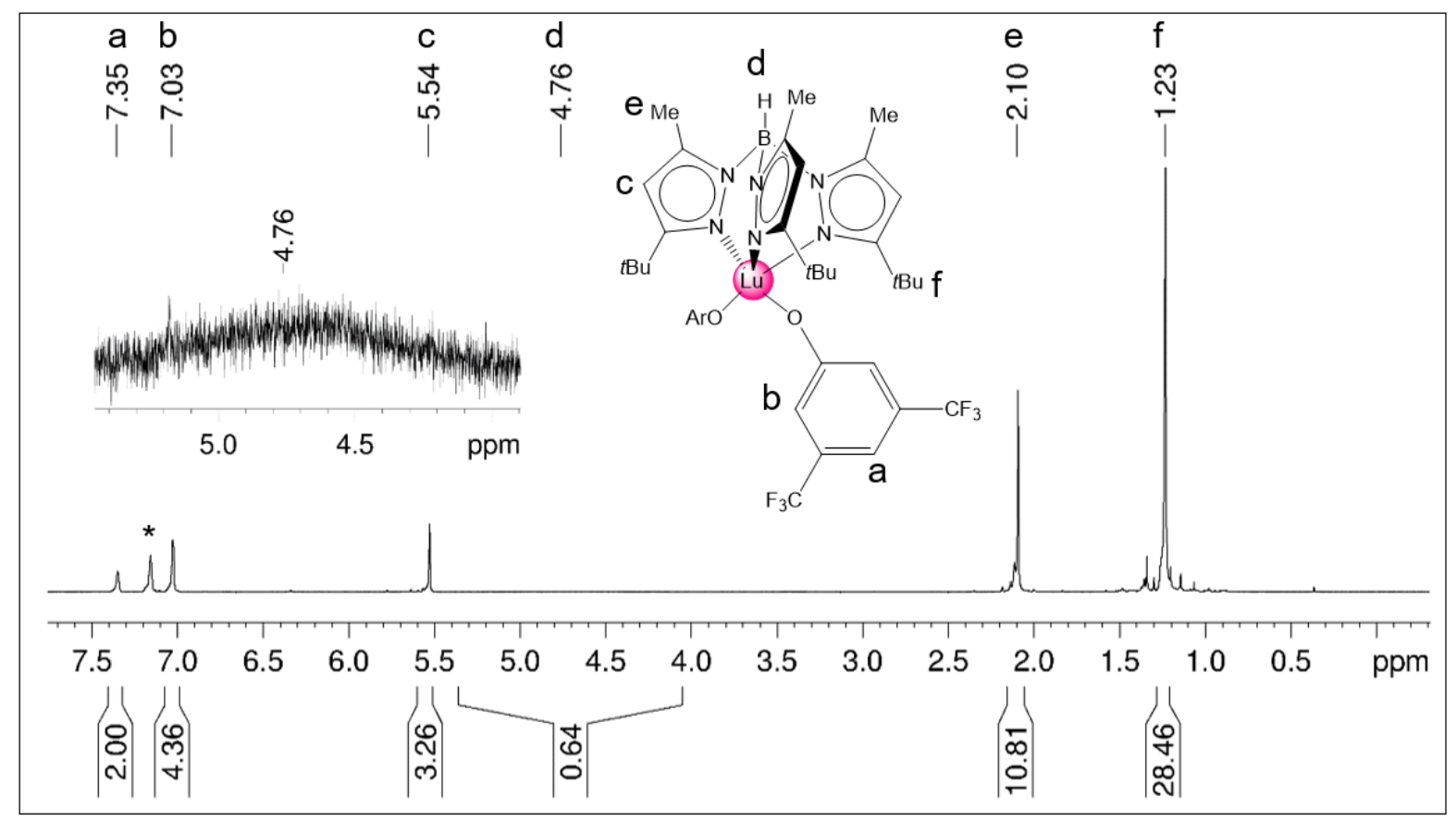

Figure S7. ${ }^{1} \mathrm{H}$ NMR spectrum (250 MHz, $\left[\mathrm{D}_{6}\right]$ benzene) of complex $\mathrm{Tp}^{\mathrm{tBu}, \mathrm{Me}} \mathrm{Lu}\left[\mathrm{OC}_{6} \mathrm{H}_{3}\left(\mathrm{CF}_{3}\right)_{2}-3,5\right]_{2}(3-\mathrm{Lu})$ at $26{ }^{\circ} \mathrm{C}$.

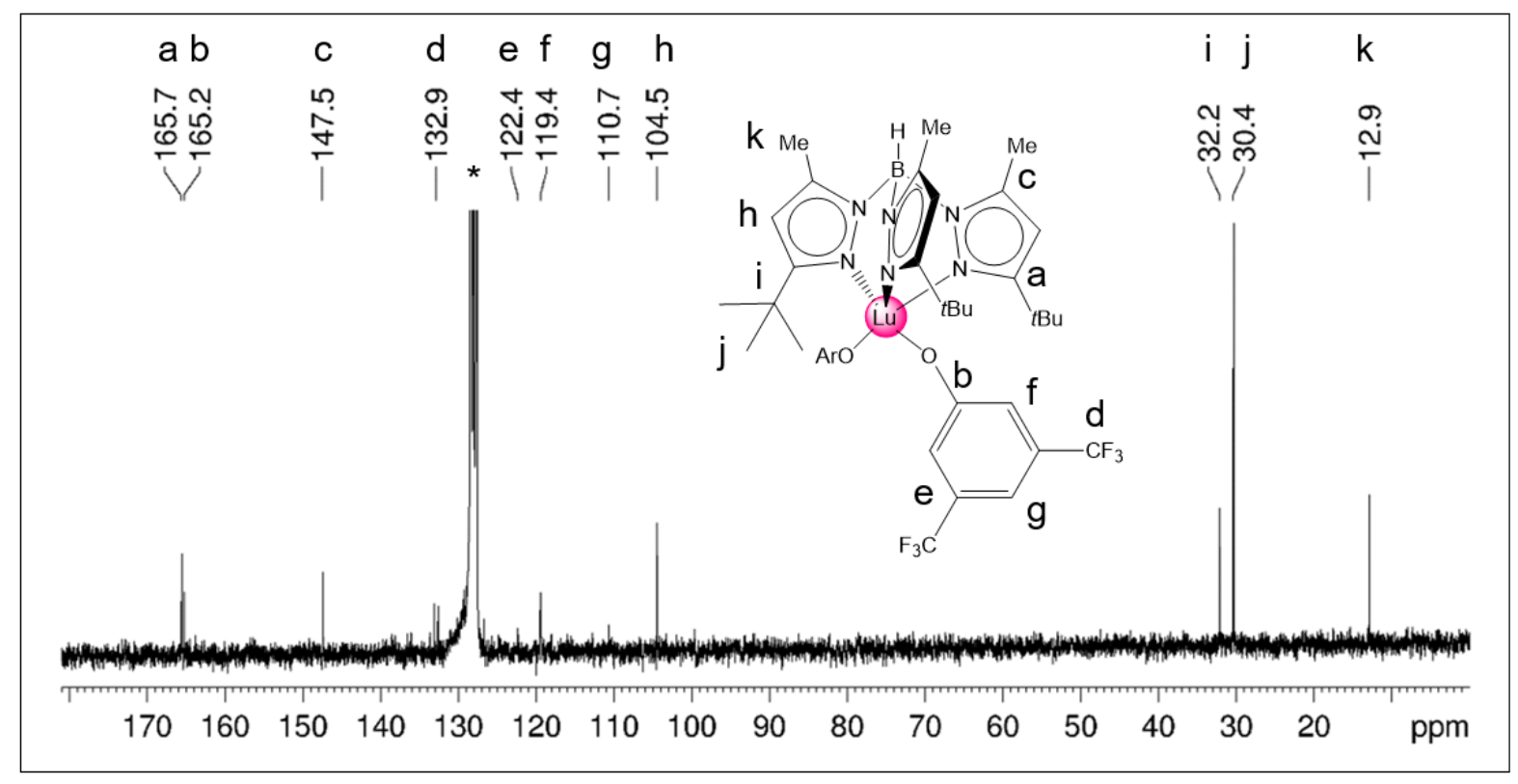

Figure S8. ${ }^{13} \mathrm{C}\left\{{ }^{1} \mathrm{H}\right\}$ NMR spectrum (63 MHz, [D6] benzene) of complex $\mathrm{Tp}^{\mathrm{tBu}, \mathrm{Me}} \mathrm{Lu}\left[\mathrm{OC}_{6} \mathrm{H}_{3}\left(\mathrm{CF}_{3}\right)_{2}-3,5\right]_{2}(3-$ Lu) at $26^{\circ} \mathrm{C}$.

Sb 


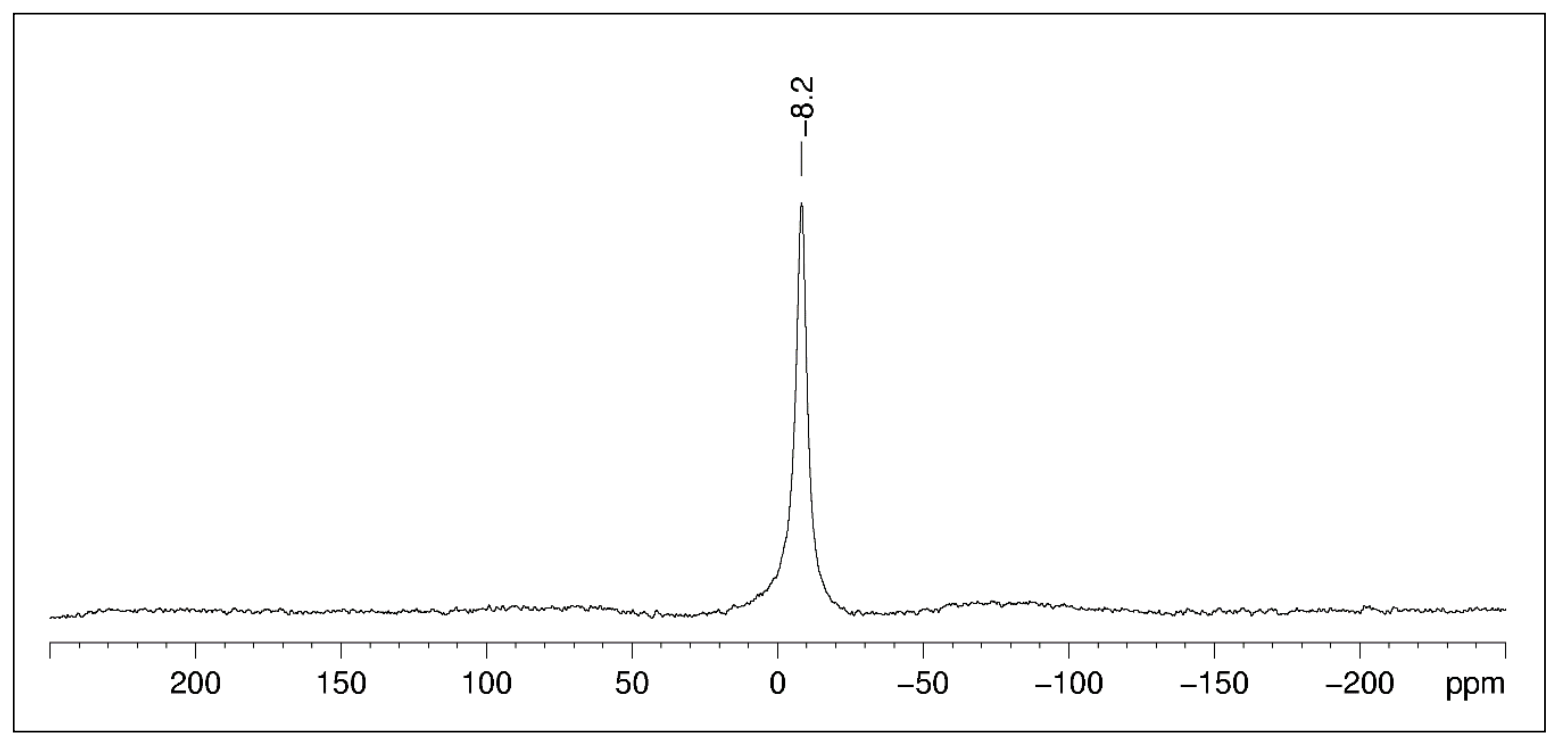

Figure S9. ${ }^{11} \mathrm{~B}\left\{{ }^{1} \mathrm{H}\right\}$ NMR spectrum $\left(80 \mathrm{MHz},\left[\mathrm{D}_{6}\right]\right.$ benzene) of complex $\mathrm{Tp}{ }^{\mathrm{tBu}, \mathrm{Me}} \mathrm{Lu}\left[\mathrm{OC}_{6} \mathrm{H}_{3}\left(\mathrm{CF}_{3}\right)_{2}-3,5\right]_{2}(3-$ Lu) at $26^{\circ} \mathrm{C}$.

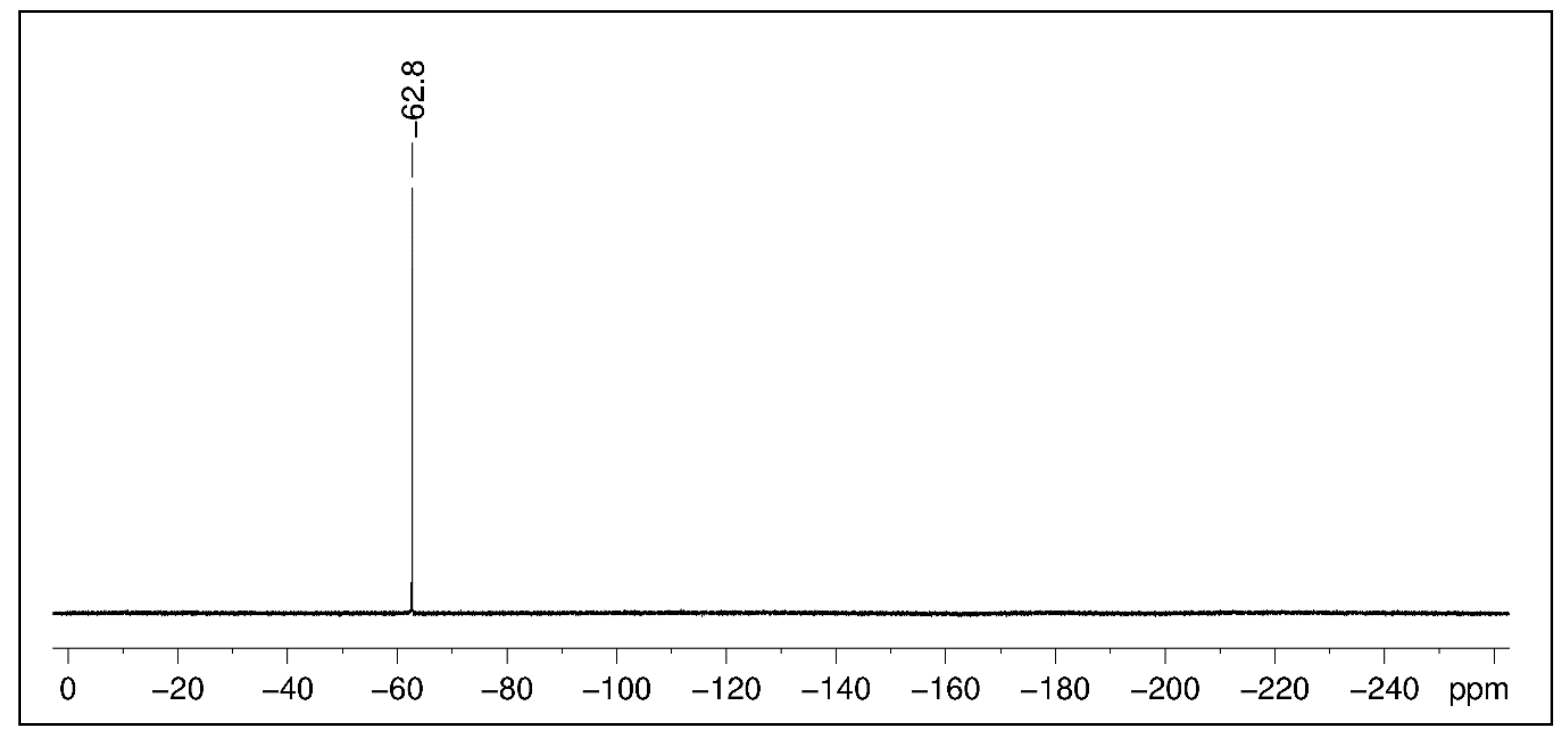

Figure S10. ${ }^{19} \mathrm{~F}\left\{{ }^{1} \mathrm{H}\right\} \mathrm{NMR}$ spectrum (376 MHz, [D $\left.\mathrm{D}_{6}\right]$ benzene) of complex $\mathrm{Tp} \mathrm{p}^{\mathrm{tBu}, \mathrm{Me}} \mathrm{Lu}\left[\mathrm{OC}_{6} \mathrm{H}_{3}\left(\mathrm{CF}_{3}\right)_{2}-3,5\right]_{2}$ (3-Lu) at $26{ }^{\circ} \mathrm{C}$. 


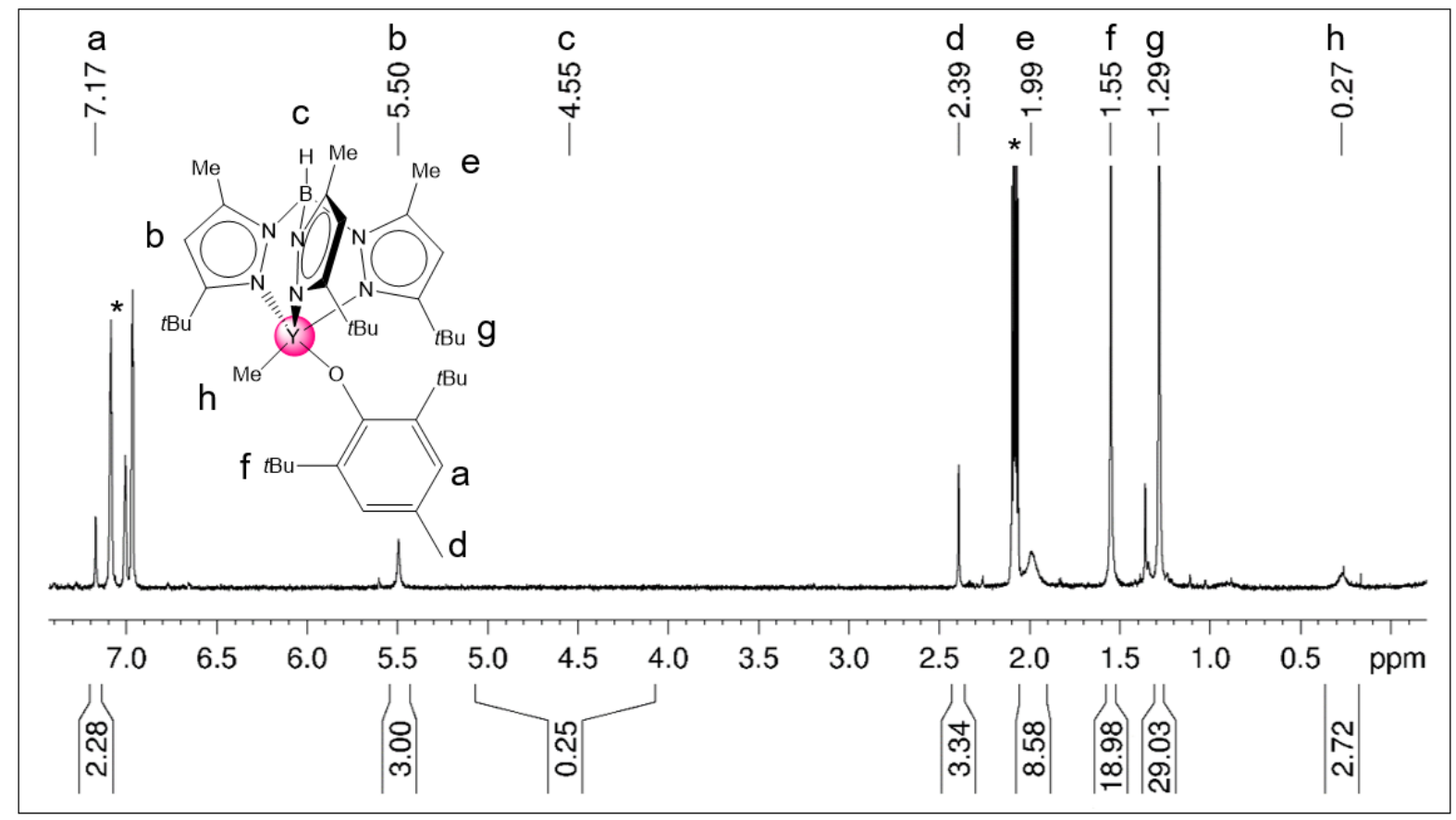

Figure S11. ${ }^{1} \mathrm{H}$ NMR spectrum $\left(500 \mathrm{MHz}\right.$, [D8 ]toluene) of complex $\mathrm{Tp}^{\mathrm{tBu}, \mathrm{Me}} \mathrm{YMe}\left(\mathrm{OC}_{6} \mathrm{H}_{2} \mathrm{BBu}_{2}-2,6-\mathrm{Me}-4\right)$ (4-Y) at $26^{\circ} \mathrm{C}$.

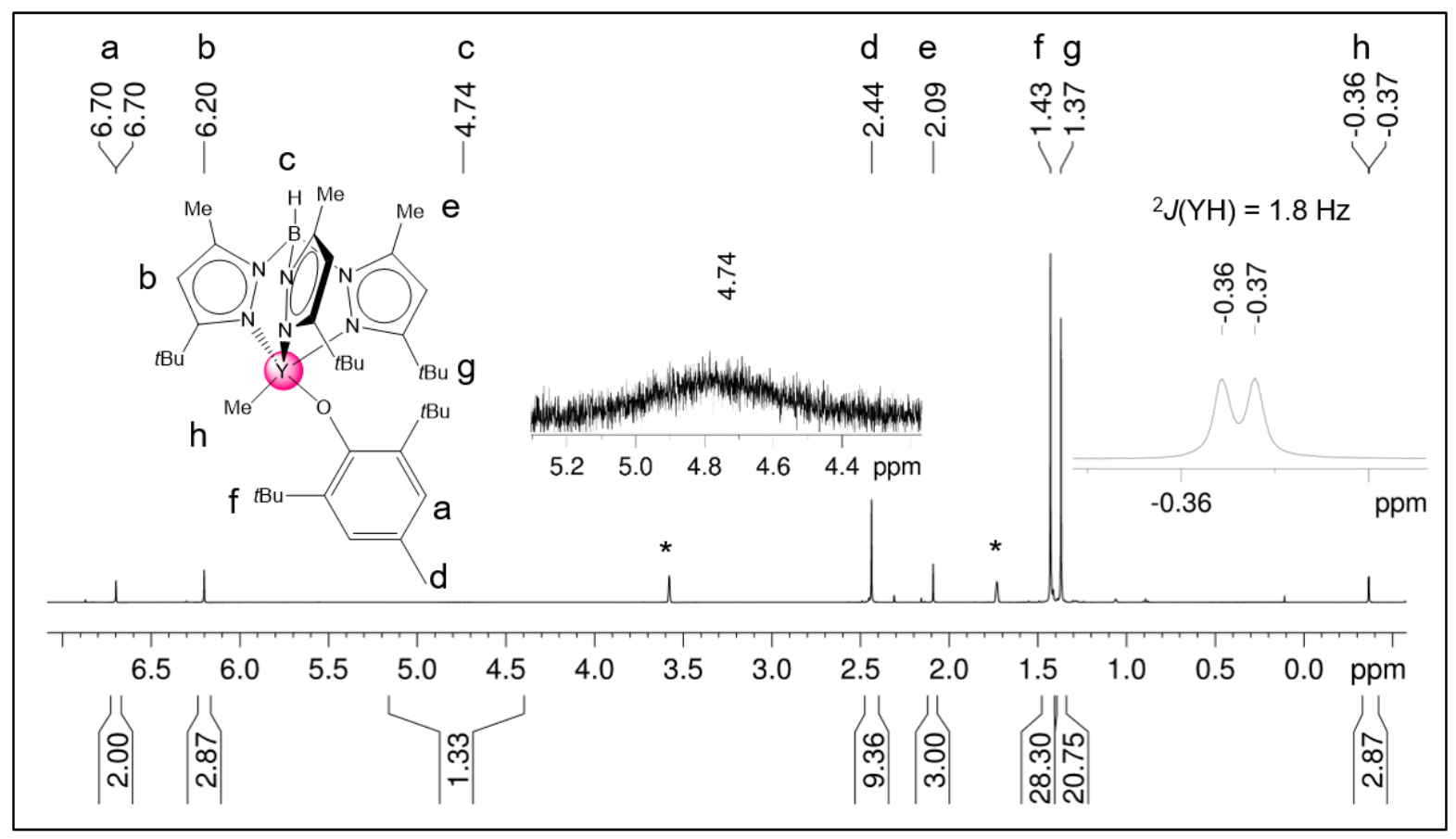

Figure S12. ${ }^{1} \mathrm{H}$ NMR spectrum (500 MHz, $\left[\mathrm{D}_{8}\right]$ the) of complex $\mathrm{Tp}^{\mathrm{tBu}, \mathrm{Me}} \mathrm{YMe}\left(\mathrm{OC}_{6} \mathrm{H}_{2} \mathrm{HBu}_{2}-2,6-\mathrm{Me}-4\right)(4-\mathrm{Y})$ at $0^{\circ} \mathrm{C}$.

SB 


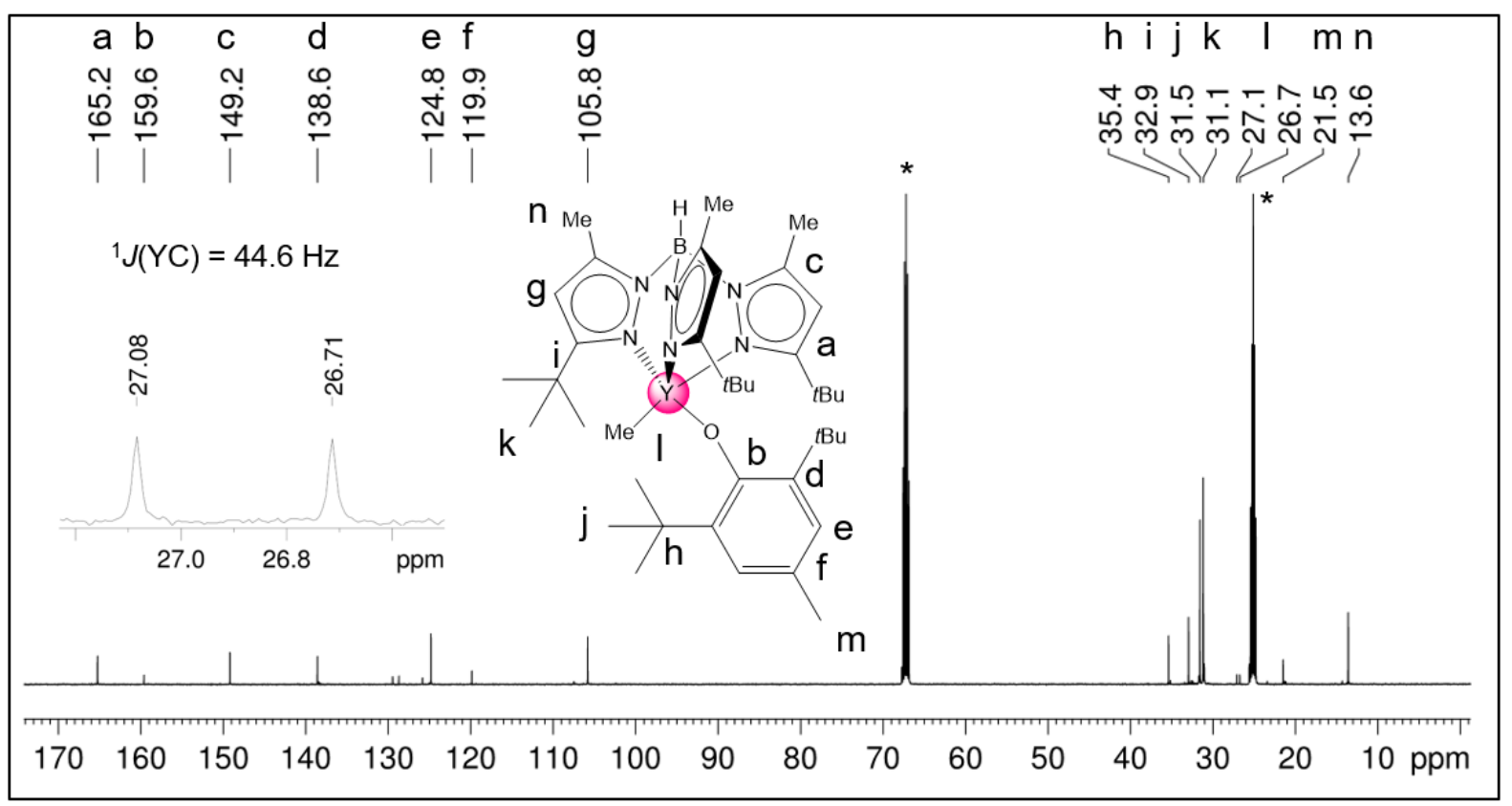

Figure S13. ${ }^{13} \mathrm{C}\left\{{ }^{1} \mathrm{H}\right\}$ NMR spectrum (126 MHz, [D8]thf) of complex $\mathrm{Tp}^{\mathrm{tBu}, \mathrm{Me}} \mathrm{YMe}\left(\mathrm{OC}_{6} \mathrm{H}_{2} t \mathrm{Bu} 2-2,6-\mathrm{Me}-4\right)$ (4-Y) at $0{ }^{\circ} \mathrm{C}$

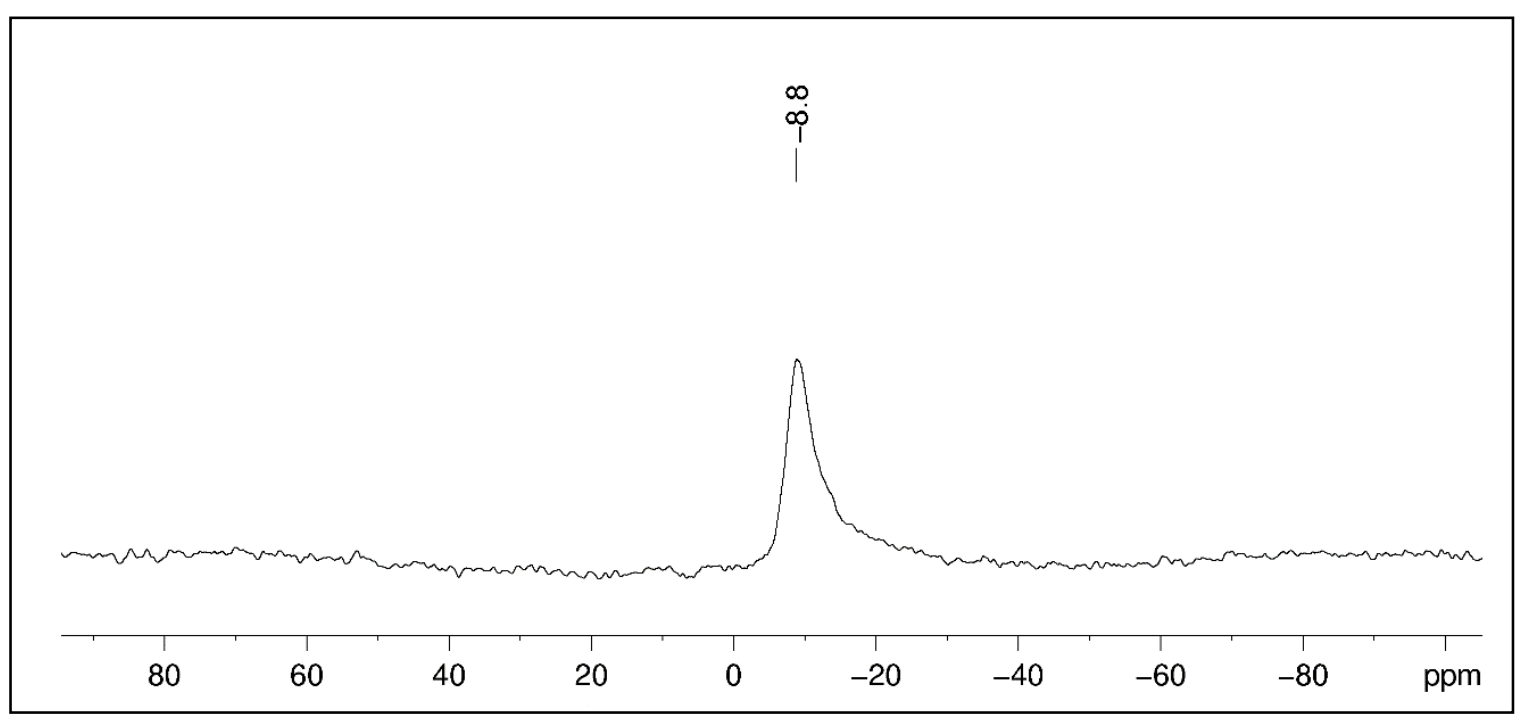

Figure S14. ${ }^{11} \mathrm{~B}\left\{{ }^{1} \mathrm{H}\right\} \mathrm{NMR}$ spectrum $\left(80 \mathrm{MHz}\right.$, [D 8 ]toluene) of complex $\mathrm{Tp}^{\mathrm{tBu}, \mathrm{Me}} \mathrm{YMe}\left(\mathrm{OC}_{6} \mathrm{H}_{2} t \mathrm{Bu}_{2}-2,6-\mathrm{Me}-\right.$ 4) $(4-Y)$ at $26{ }^{\circ} \mathrm{C}$. 


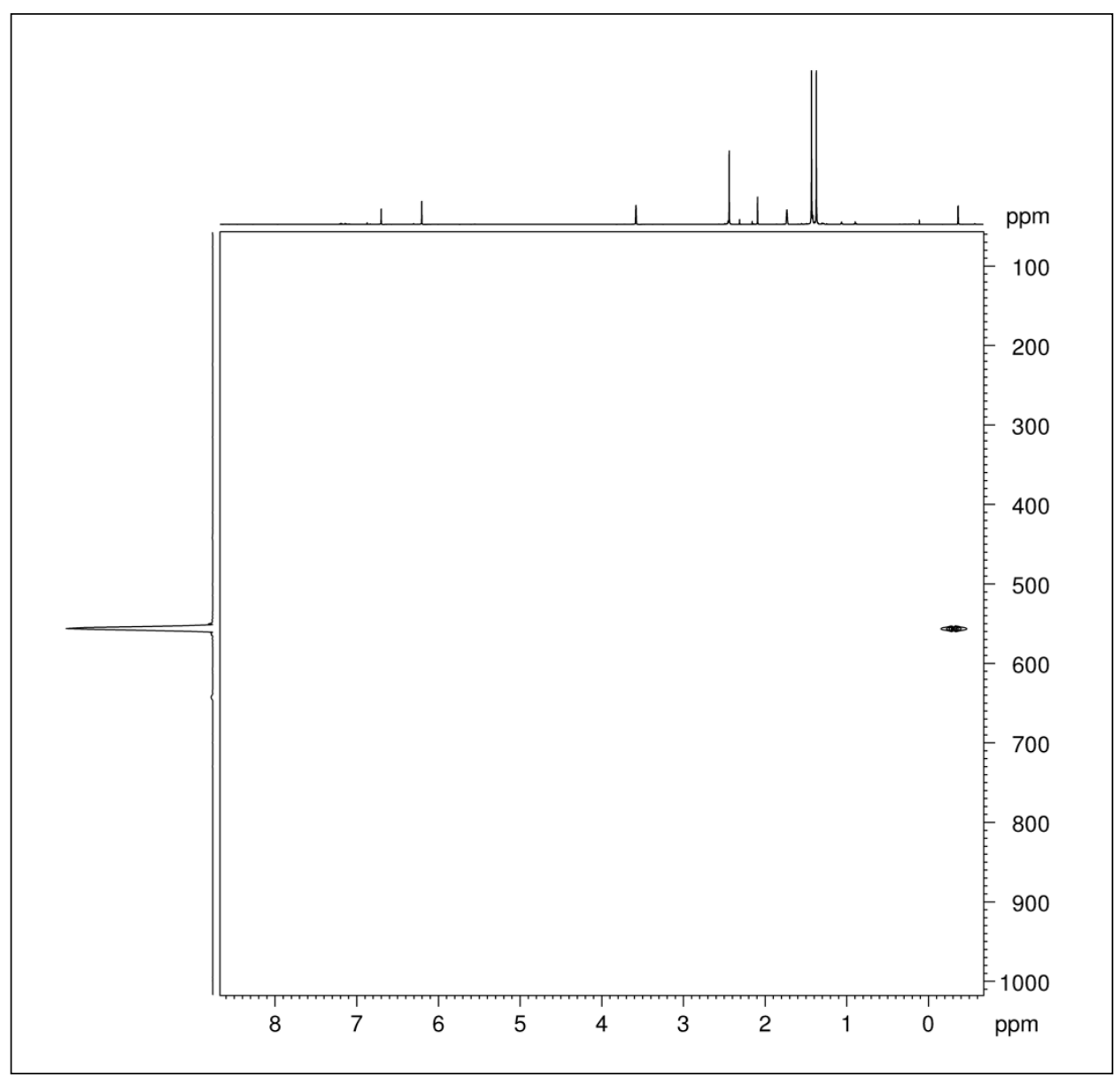

Figure S15. ${ }^{1} \mathrm{H}^{8}{ }^{89} \mathrm{Y} \mathrm{HSQC}$ NMR spectrum $\left(25 \mathrm{MHz}\right.$, [D 8 ]thf) of complex $\mathrm{Tp}^{\mathrm{tBu}, \mathrm{Me}} \mathrm{YMe}\left(\mathrm{OC}_{6} \mathrm{H}_{2} t \mathrm{Bu}_{2}-2,6-\mathrm{Me}-\right.$ 4) (4-Y) at $0{ }^{\circ} \mathrm{C}$.

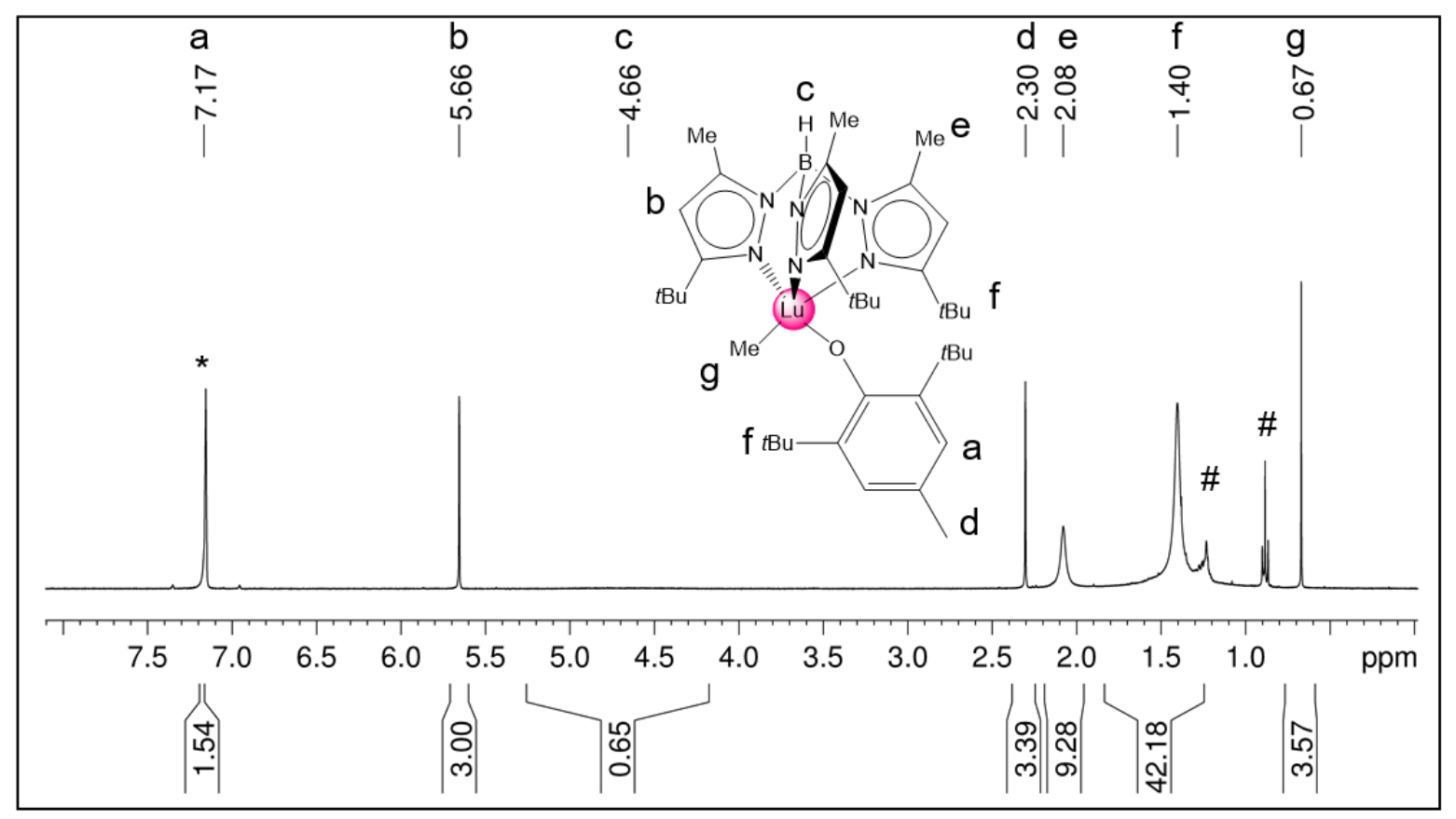

Figure S16. ${ }^{1} \mathrm{H}$ NMR spectrum $\left(400 \mathrm{MHz}\right.$, $\left[\mathrm{D}_{6}\right]$ benzene) of complex $\mathrm{Tp}^{\mathrm{tBu}, \mathrm{Me}} \mathrm{LuMe}\left(\mathrm{OC}_{6} \mathrm{H}_{2} t \mathrm{Bu} \mathrm{L}_{2}-2,6-\mathrm{Me}-\right.$ 4) (4-Lu) at $26^{\circ} \mathrm{C}$ with traces of $n$-hexane (\#). 


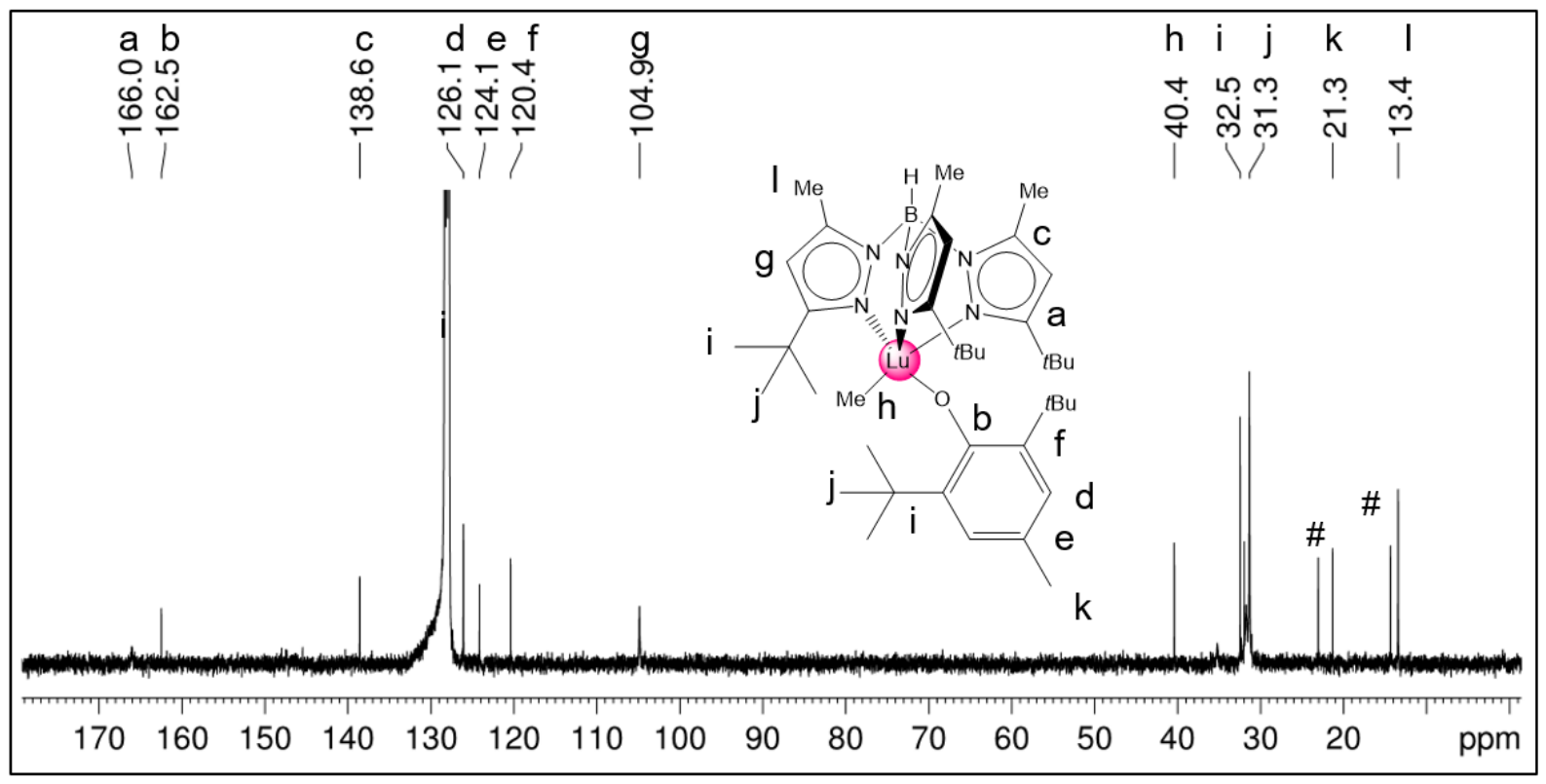

Figure S17. ${ }^{13} \mathrm{C}\left\{{ }^{1} \mathrm{H}\right\}$ NMR spectrum (101 MHz, $\left[\mathrm{D}_{6}\right]$ benzene) of complex Tp ${ }^{\mathrm{tBu}, \mathrm{Me}} \mathrm{LuMe}\left(\mathrm{OC}_{6} \mathrm{H}_{2} \mathrm{ABu}_{2}-2,6-\right.$ Me-4) (4-Lu) at $26{ }^{\circ} \mathrm{C}$ with traces of $n$-hexane (\#).

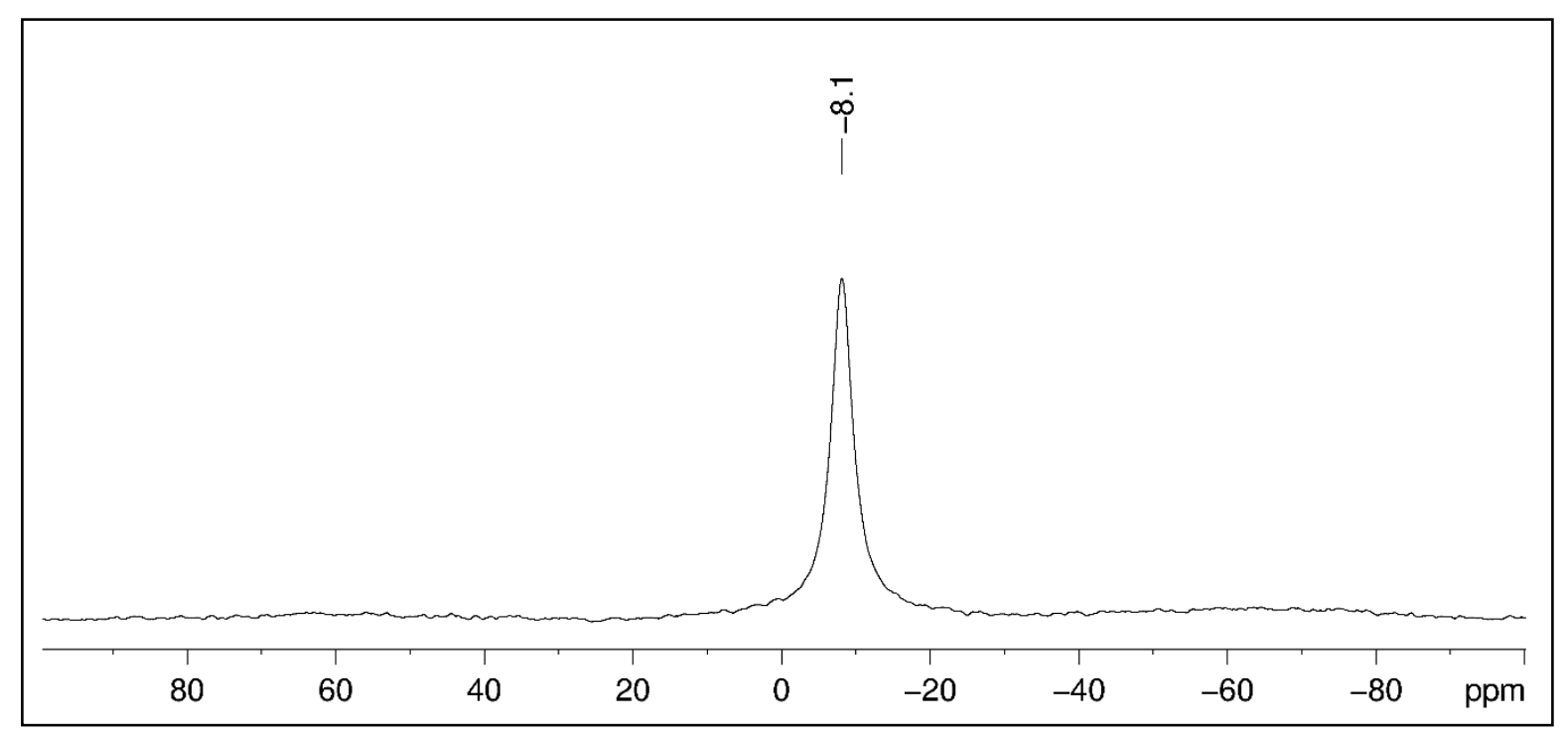

Figure S18. ${ }^{11} \mathrm{~B}\left\{{ }^{1} \mathrm{H}\right\}$ NMR spectrum $\left(80 \mathrm{MHz}\right.$, $\left[\mathrm{D}_{6}\right]$ benzene) of complex $\mathrm{Tp} \mathrm{p}^{\mathrm{tBu}, \mathrm{Me}} \mathrm{LuMe}\left(\mathrm{OC}_{6} \mathrm{H}_{2} t \mathrm{Bu} \mathrm{C}_{2}-2,6-\right.$ Me-4) (4-Lu) at $26^{\circ} \mathrm{C}$. 


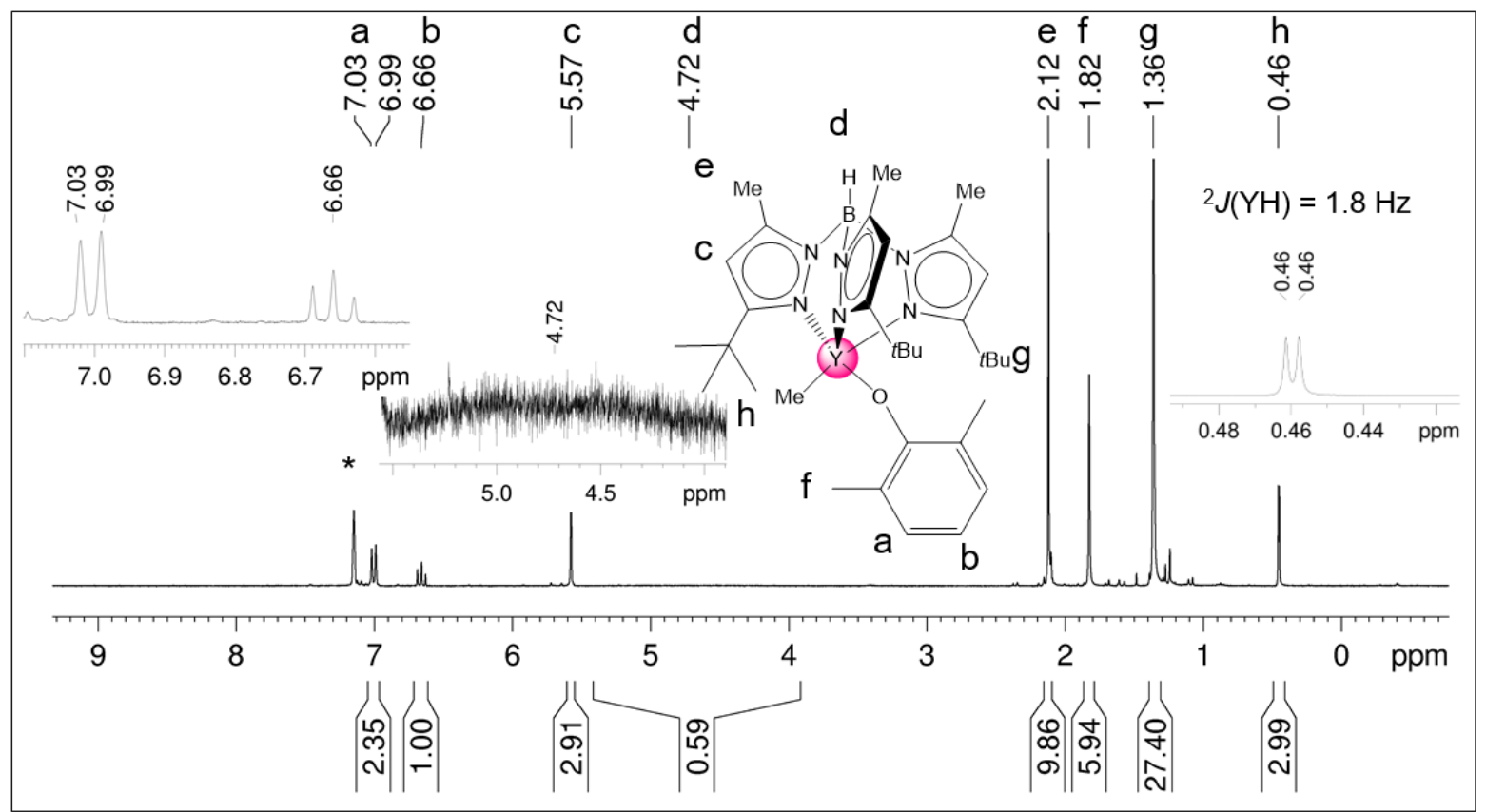

Figure S19. ${ }^{1} \mathrm{H}$ NMR spectrum $\left(250 \mathrm{MHz},\left[\mathrm{D}_{6}\right]\right.$ benzene) of complex $\mathrm{Tp}^{\mathrm{tBu}, \mathrm{Me}} \mathrm{YMe}\left(\mathrm{OC}_{6} \mathrm{H}_{3} \mathrm{Me}_{2}-2,6\right)(5-Y)$ at $26{ }^{\circ} \mathrm{C}$.

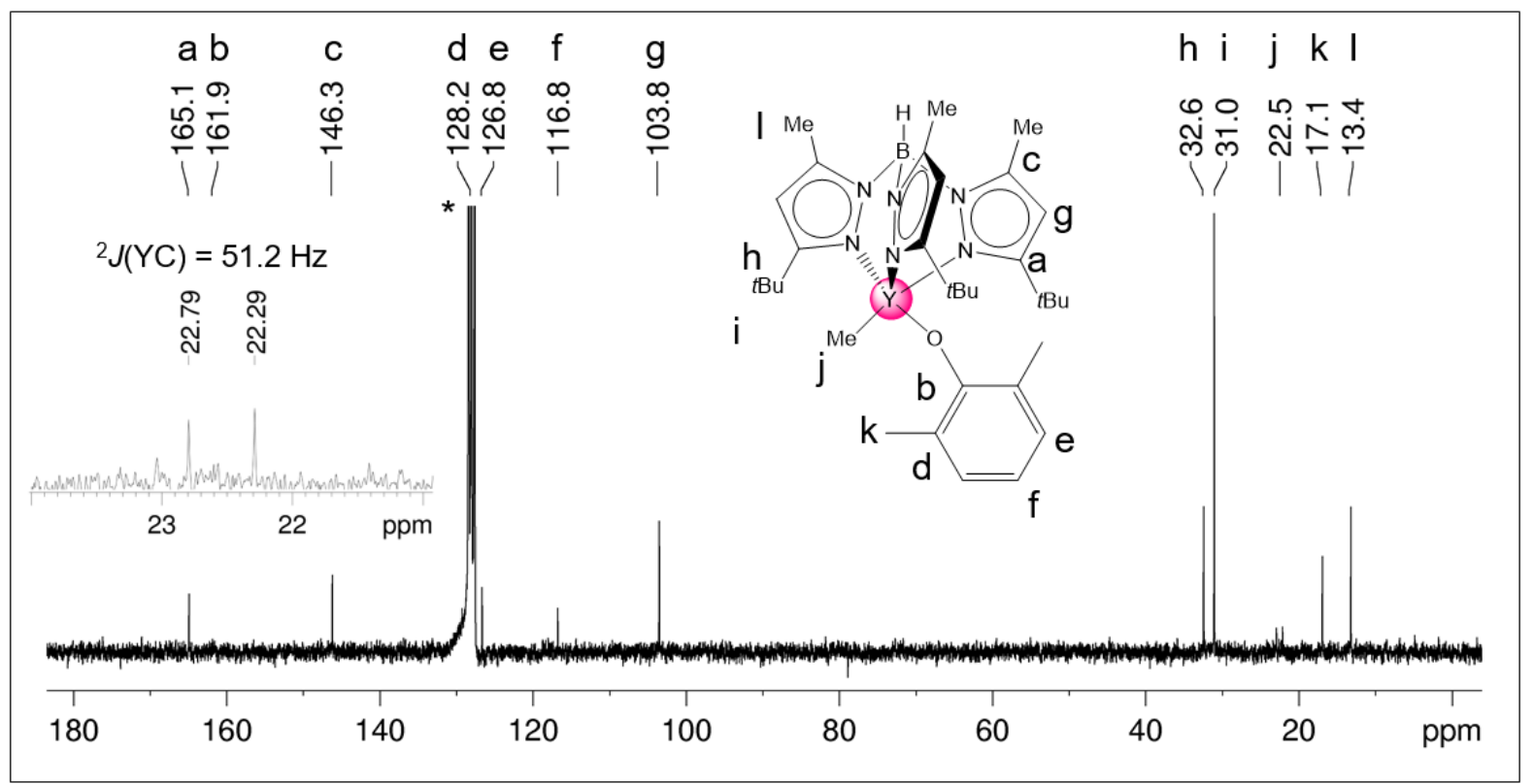

Figure S20. ${ }^{13} \mathrm{C}\left\{{ }^{1} \mathrm{H}\right\} \mathrm{NMR}$ spectrum $\left(63 \mathrm{MHz}\right.$, [D6]benzene) of complex $\mathrm{Tp} \mathrm{D}^{\mathrm{tBu}, \mathrm{Me}} \mathrm{YMe}\left(\mathrm{OC}_{6} \mathrm{H}_{3} \mathrm{Me}_{2}-2,6\right)(\mathbf{5 -}$ Y) at $26^{\circ} \mathrm{C}$. 


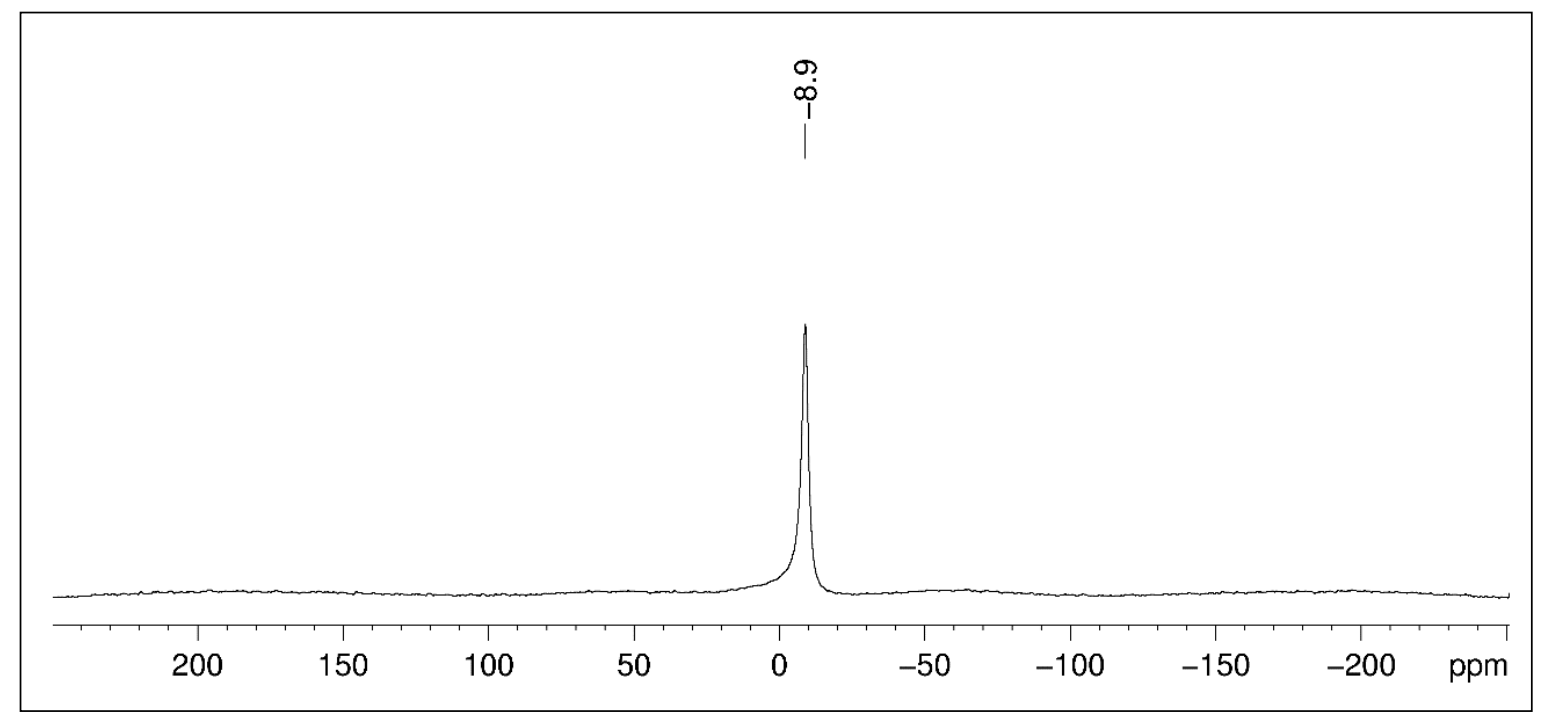

Figure S21. ${ }^{11} \mathrm{~B}\left\{{ }^{1} \mathrm{H}\right\}$ NMR spectrum (80 MHz, [D6]benzene) of complex Tp ${ }^{t \mathrm{Bu}, \mathrm{Me}} \mathrm{YMe}\left(\mathrm{OC}_{6} \mathrm{H}_{3} \mathrm{Me}_{2}-2,6\right)(5-$ Y) at $26^{\circ} \mathrm{C}$.

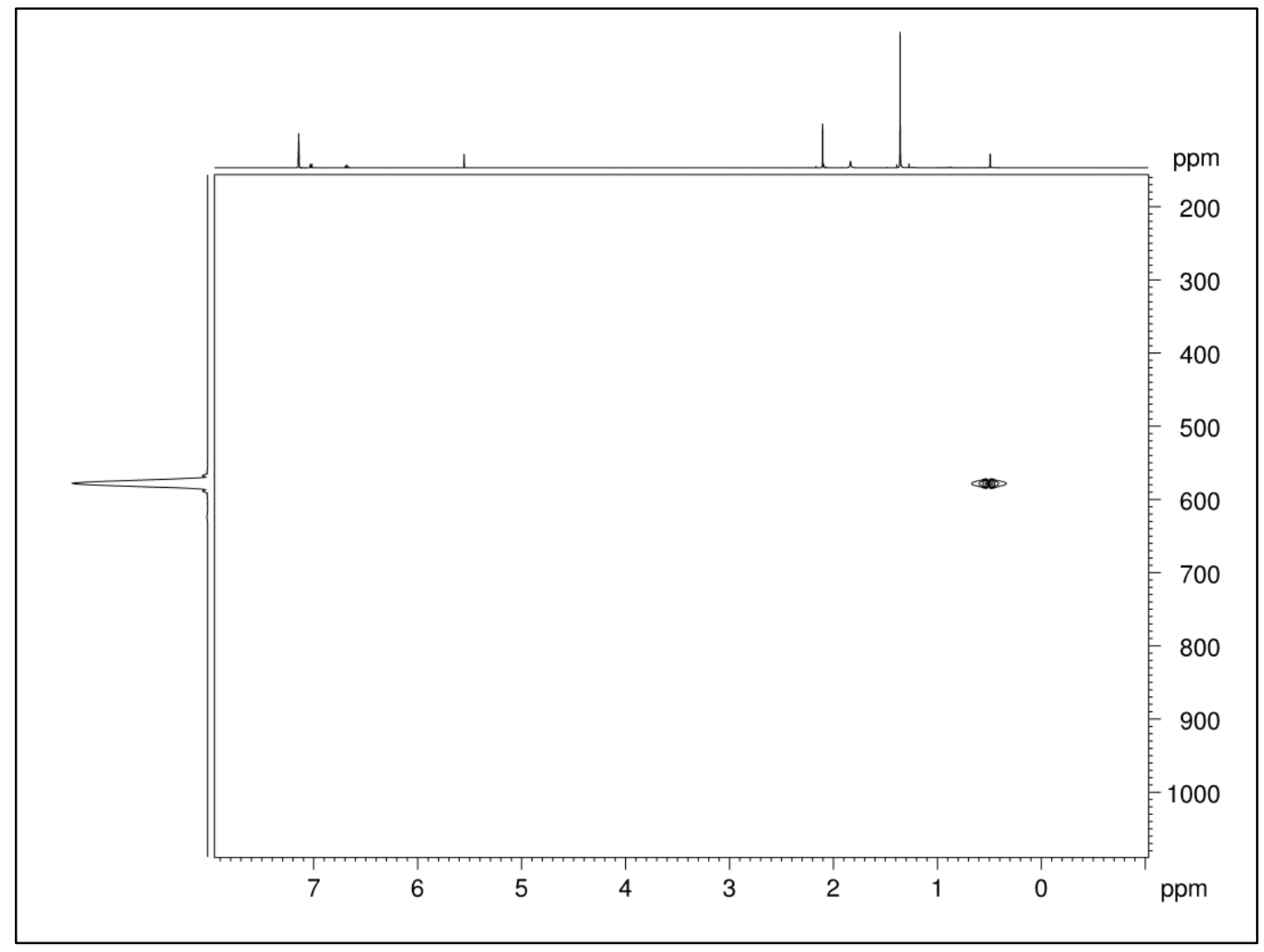

Figure S22. ${ }^{1} \mathrm{H}^{89}{ }^{8} \mathrm{YSQC}$ NMR spectrum $\left(25 \mathrm{MHz}\right.$, [D6]benzene) of complex $\mathrm{Tp}^{\mathrm{tBu}, \mathrm{Me}} \mathrm{YMe}_{6}\left(\mathrm{OC}_{6} \mathrm{H}_{3} \mathrm{Me}_{2}-\right.$ $2,6)(5-Y)$ at $26^{\circ} \mathrm{C}$. 


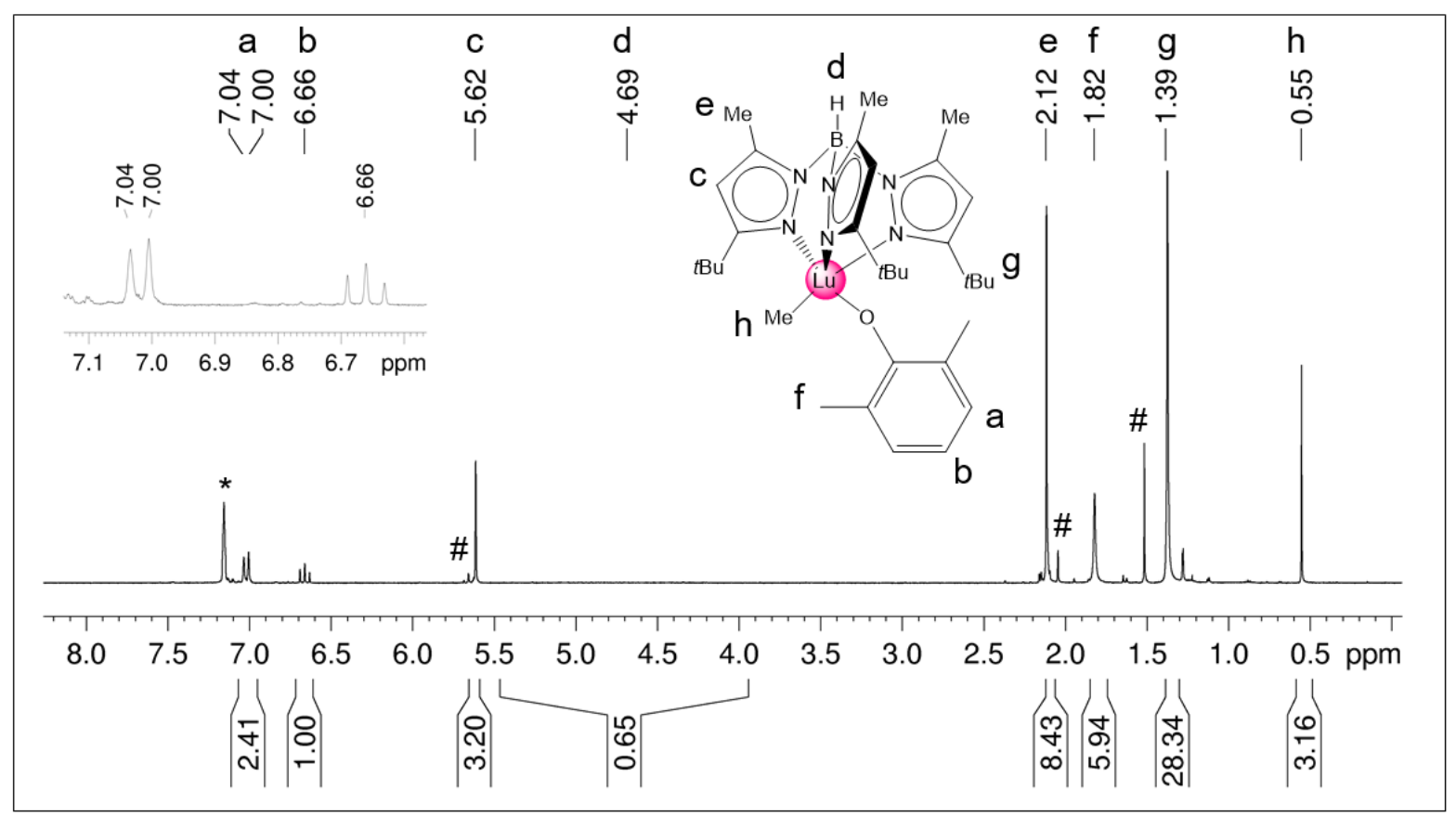

Figure S23. ${ }^{1} \mathrm{H}$ NMR spectrum $\left(250 \mathrm{MHz},\left[\mathrm{D}_{6}\right]\right.$ benzene) of complex $\mathrm{Tp}^{\mathrm{tBu}, \mathrm{Me}} \mathrm{LuMe}\left(\mathrm{OC}_{6} \mathrm{H}_{3} \mathrm{Me}_{2}-2,6\right)(5-$ $\mathrm{Lu})$ at $26^{\circ} \mathrm{C}$ with traces of $\mathrm{Tp}^{\mathrm{tB} u, \mathrm{Me}} \mathrm{LuMe}\left(\mathrm{AlMe}_{4}\right)(\#)$.

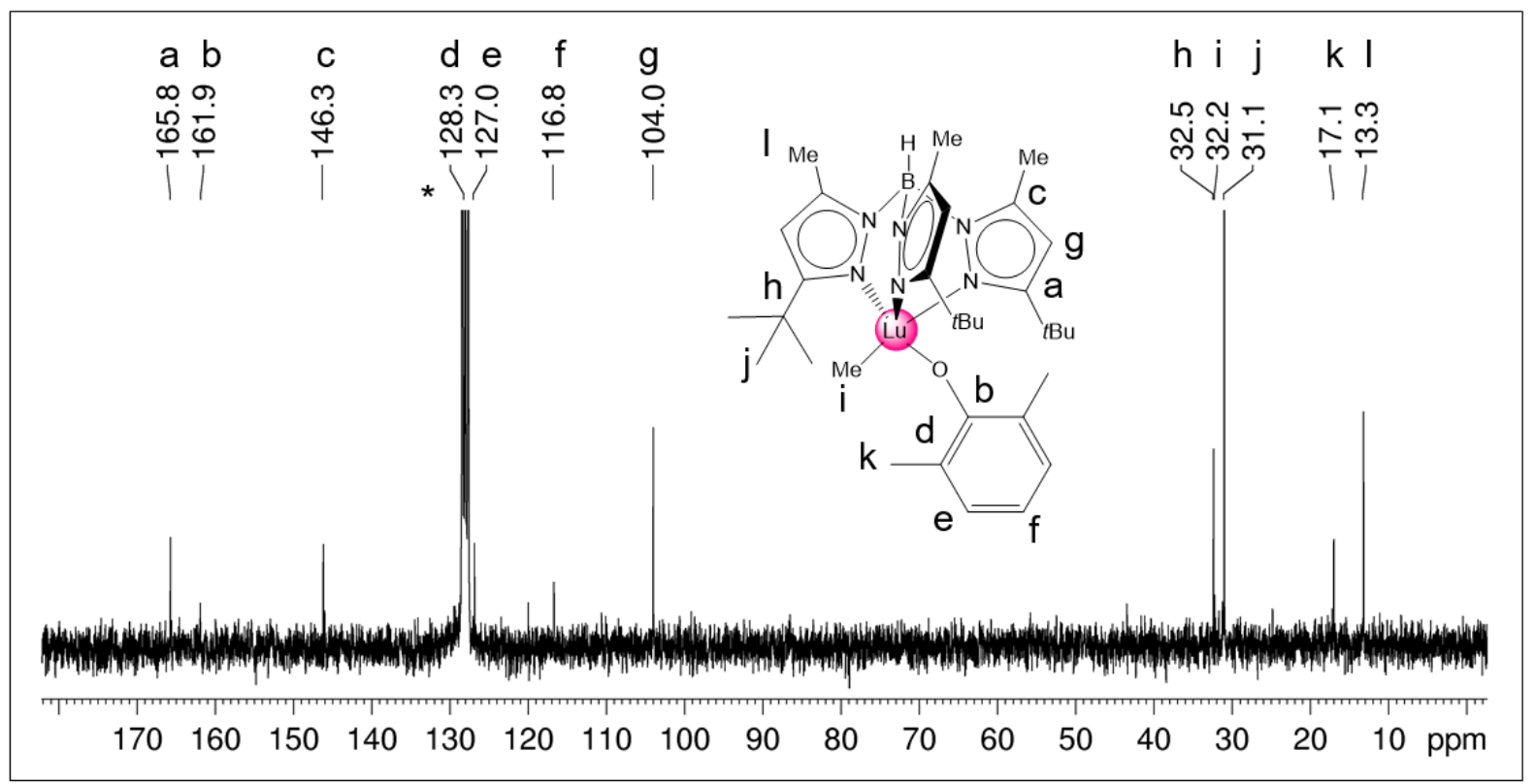

Figure S24. ${ }^{13} \mathrm{C}\left\{{ }^{1} \mathrm{H}\right\}$ NMR spectrum (63 MHz, [D $\left.\mathrm{D}_{6}\right]$ benzene) of complex $\mathrm{Tp} \mathrm{p}^{\mathrm{tBu}, \mathrm{Me}} \mathrm{LuMe}\left(\mathrm{OC}_{6} \mathrm{H}_{3} \mathrm{Me}_{2}-2,6\right)$ (5-Lu) at $26{ }^{\circ} \mathrm{C}$. 


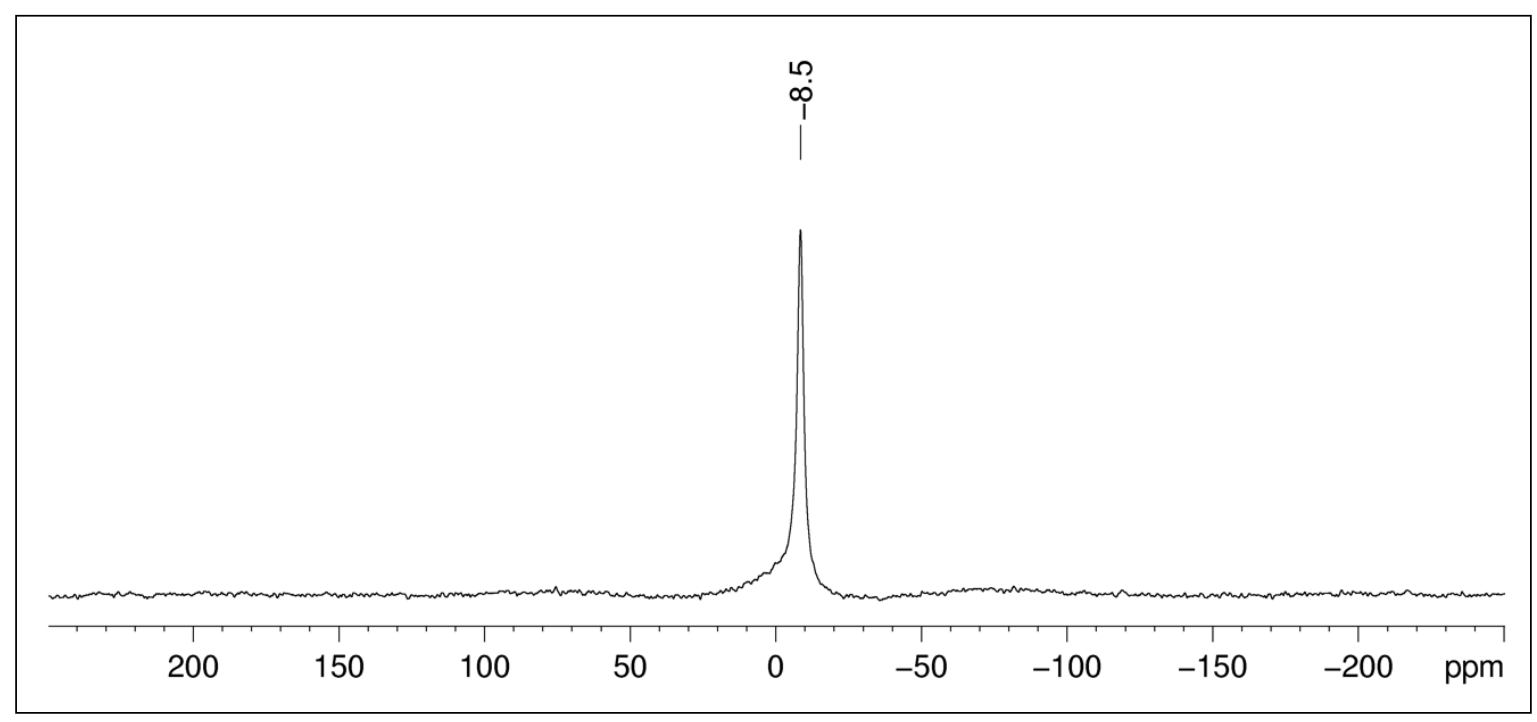

Figure S25. ${ }^{11} \mathrm{~B}\left\{{ }^{1} \mathrm{H}\right\}$ NMR spectrum (80 MHz, $\left[\mathrm{D}_{6}\right]$ benzene) of complex $\mathrm{Tp} \mathrm{t}^{\mathrm{tB}, \mathrm{Me}} \mathrm{LuMe}\left(\mathrm{OC}_{6} \mathrm{H}_{3} \mathrm{Me}_{2}-2,6\right)(5-$ Lu) at $26^{\circ} \mathrm{C}$.

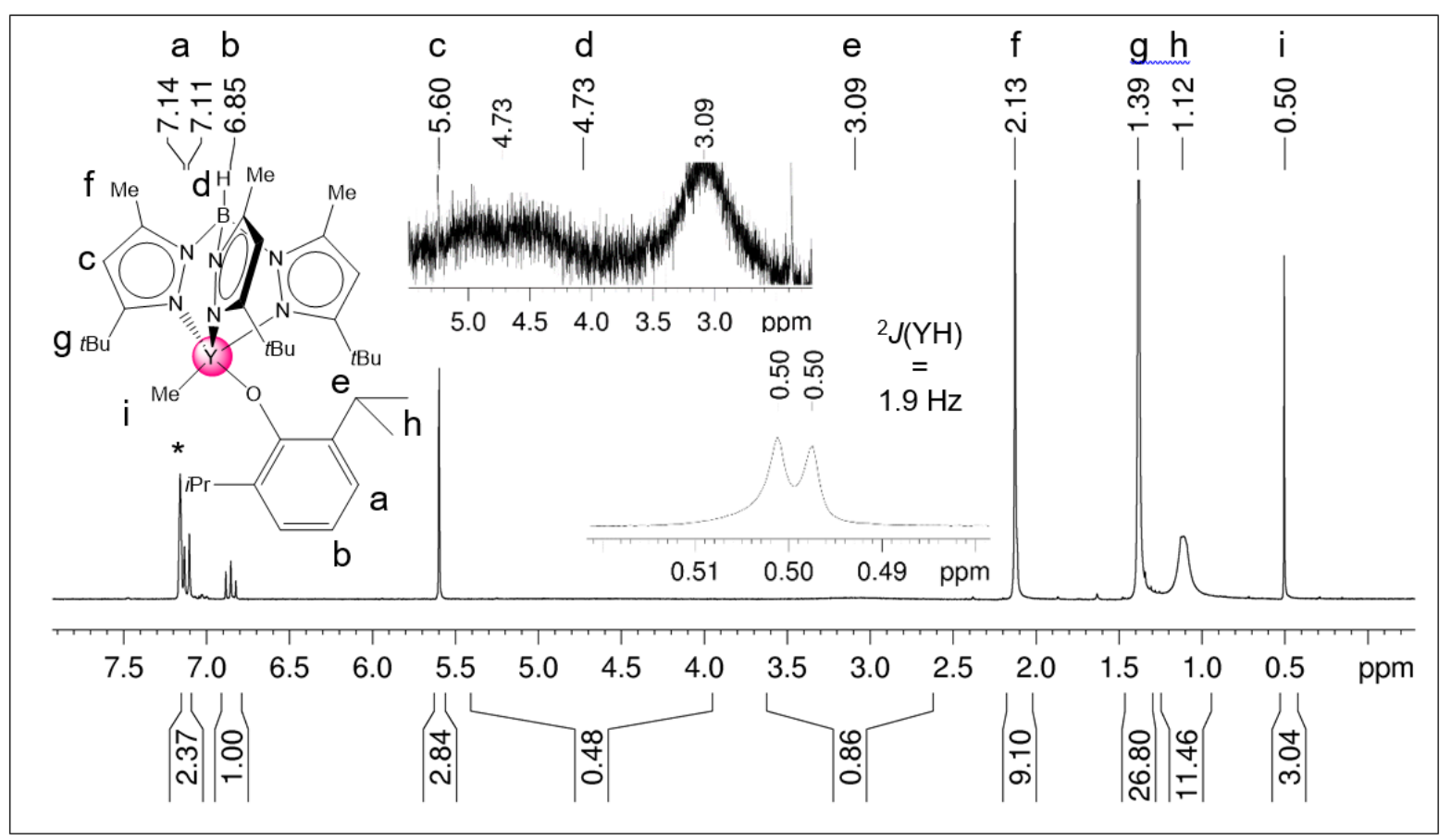

Figure S26. ${ }^{1} \mathrm{H}$ NMR spectrum (250 MHz, [D $\left.\mathrm{D}_{6}\right]$ benzene) of complex $\mathrm{Tp}^{\mathrm{tBu}, \mathrm{Me}} \mathrm{YMe}\left(\mathrm{OC}_{6} \mathrm{H}_{3} / \mathrm{Pr}_{2}-2,6\right)(6-\mathrm{Y})$ at $26{ }^{\circ} \mathrm{C}$. 


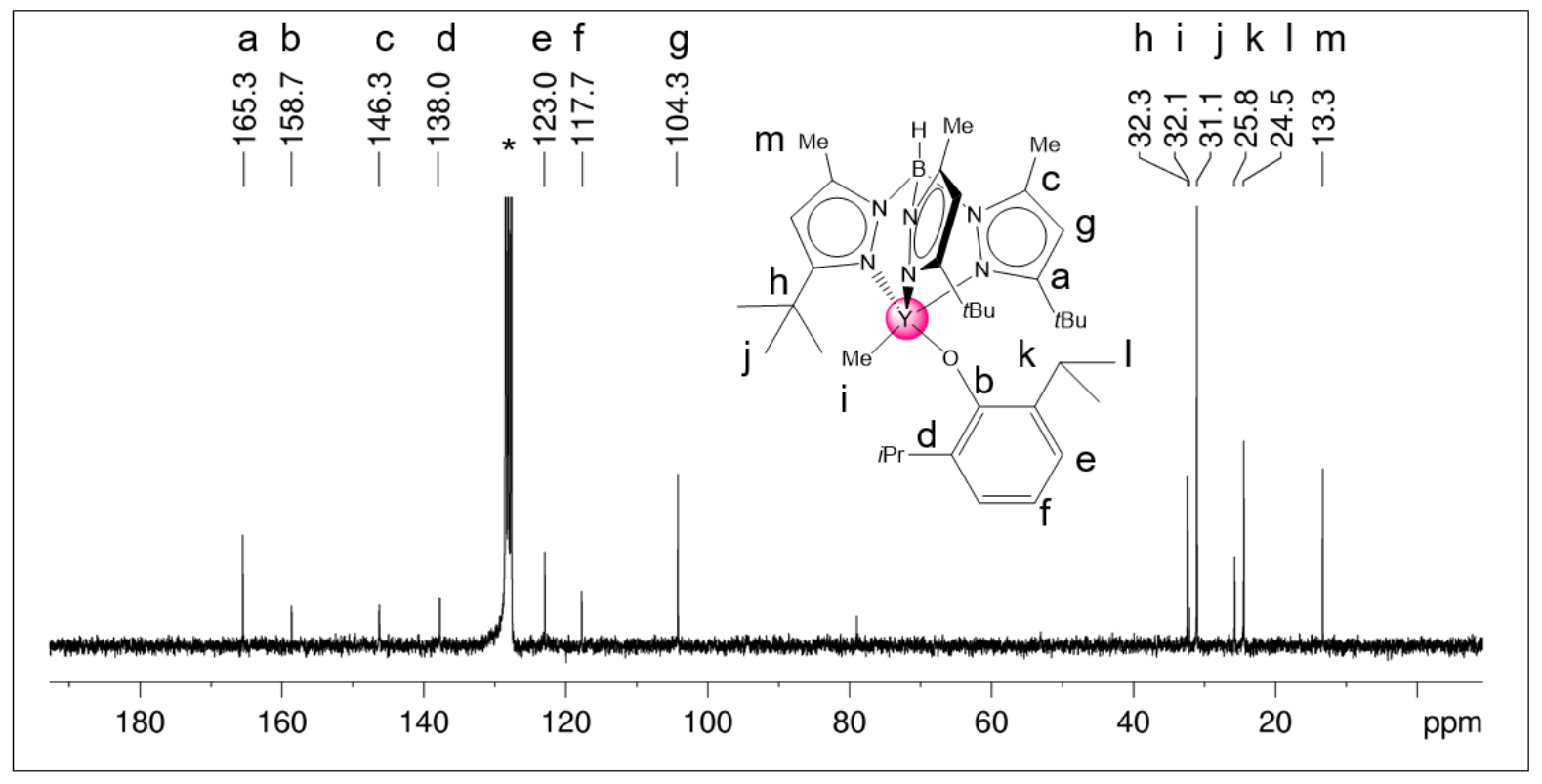

Figure S27. ${ }^{13} \mathrm{C}\left\{{ }^{1} \mathrm{H}\right\}$ NMR spectrum (63 MHz, $\left[\mathrm{D}_{6}\right]$ benzene) of complex $\mathrm{Tp}^{\mathrm{ABu}, \mathrm{Me} Y M e}\left(\mathrm{OC}_{6} \mathrm{H}_{3} i \mathrm{Pr}_{2}-2,6\right)$ (6-Y) at $26{ }^{\circ} \mathrm{C}$, no Y-C coupling was observed.

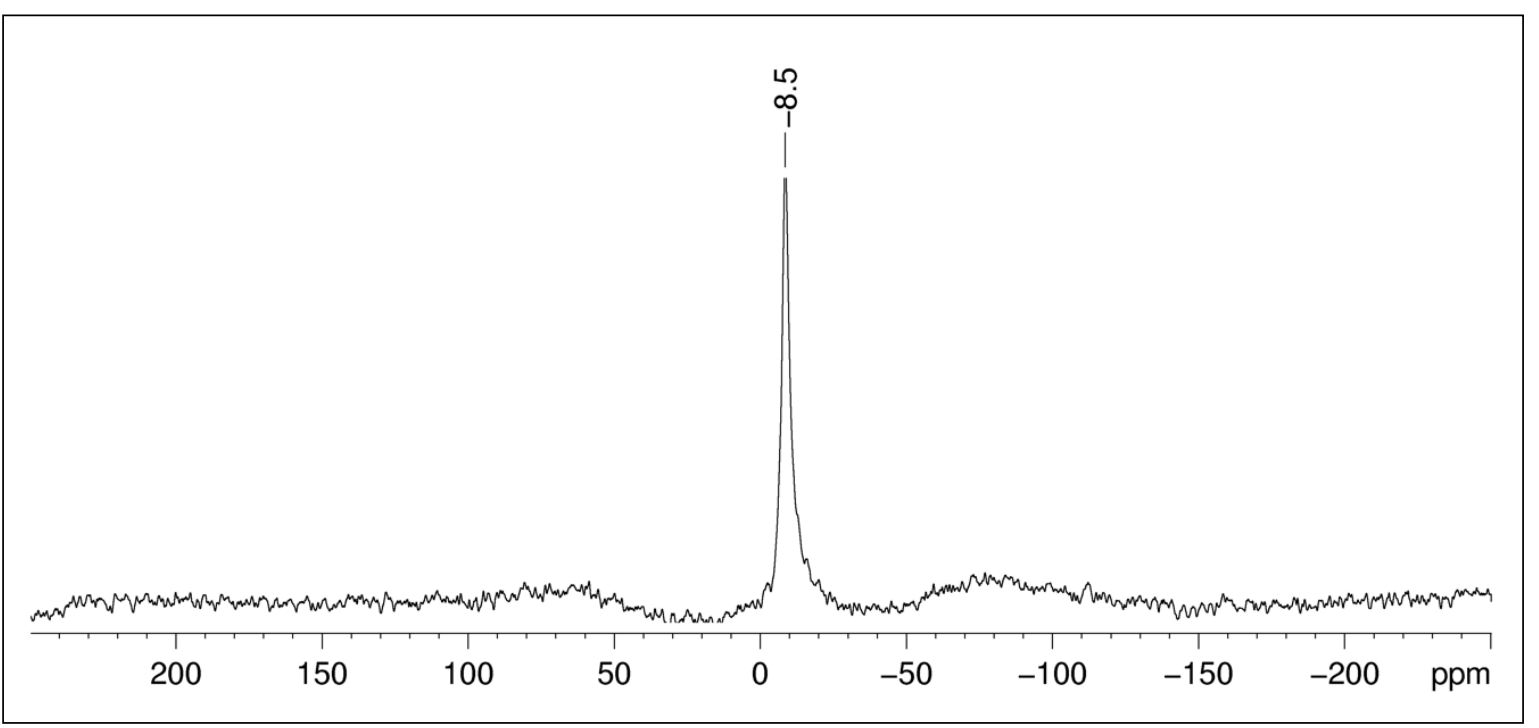

Figure S28. ${ }^{11} \mathrm{~B}\left\{{ }^{1} \mathrm{H}\right\} \mathrm{NMR}$ spectrum (80 MHz, [D6]benzene) of complex $\mathrm{Tp}^{\mathrm{tB} u, \mathrm{Me}} \mathrm{YMe}\left(\mathrm{OC}_{6} \mathrm{H}_{3} \mathrm{PPr}_{2}-2,6\right)(6-$ Y) at $26^{\circ} \mathrm{C}$. 


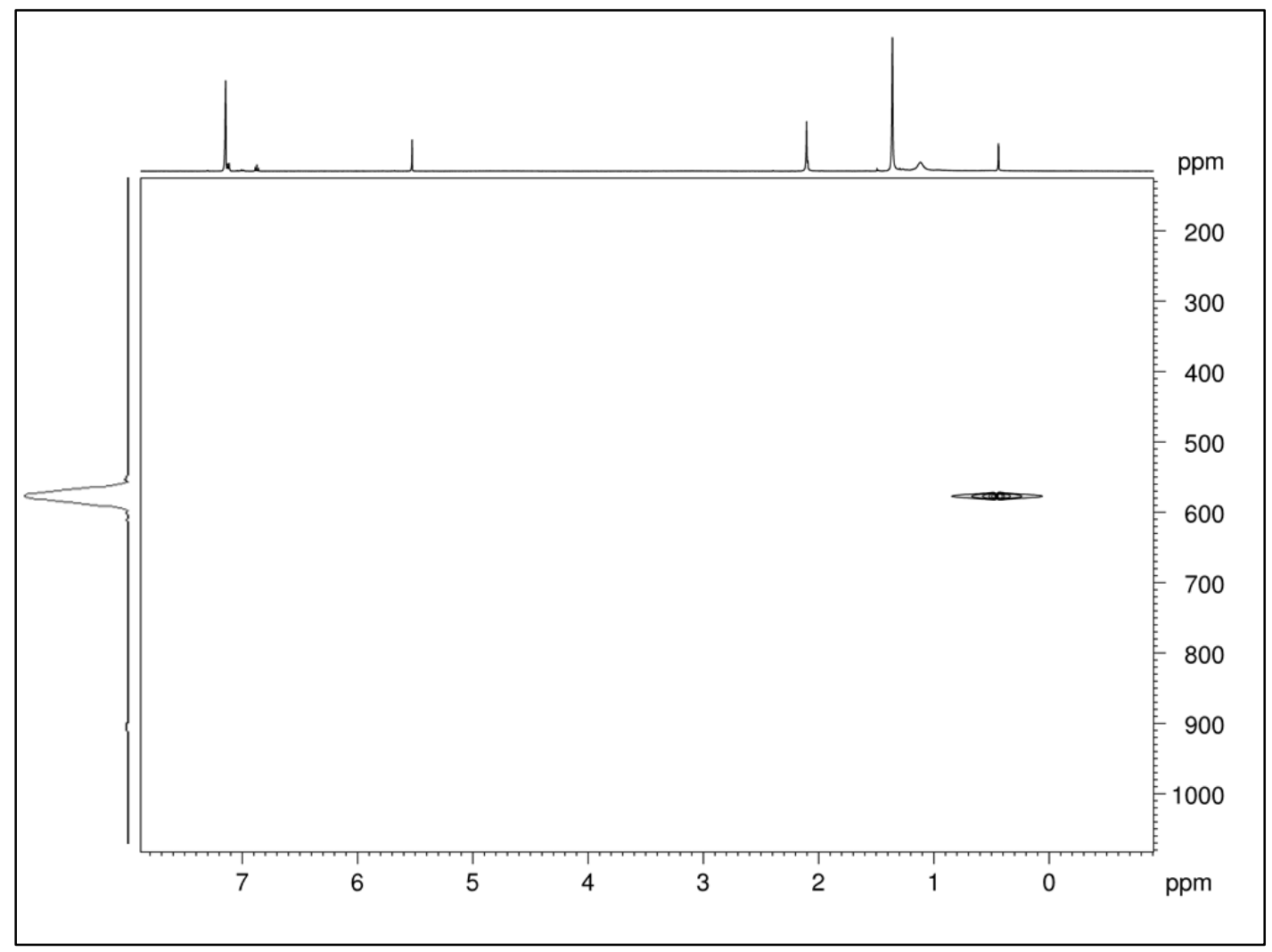

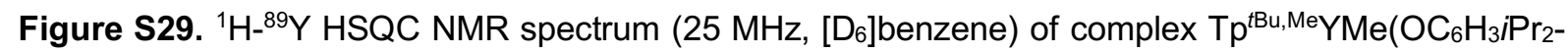
2,6) (6-Y) at $26^{\circ} \mathrm{C}$.

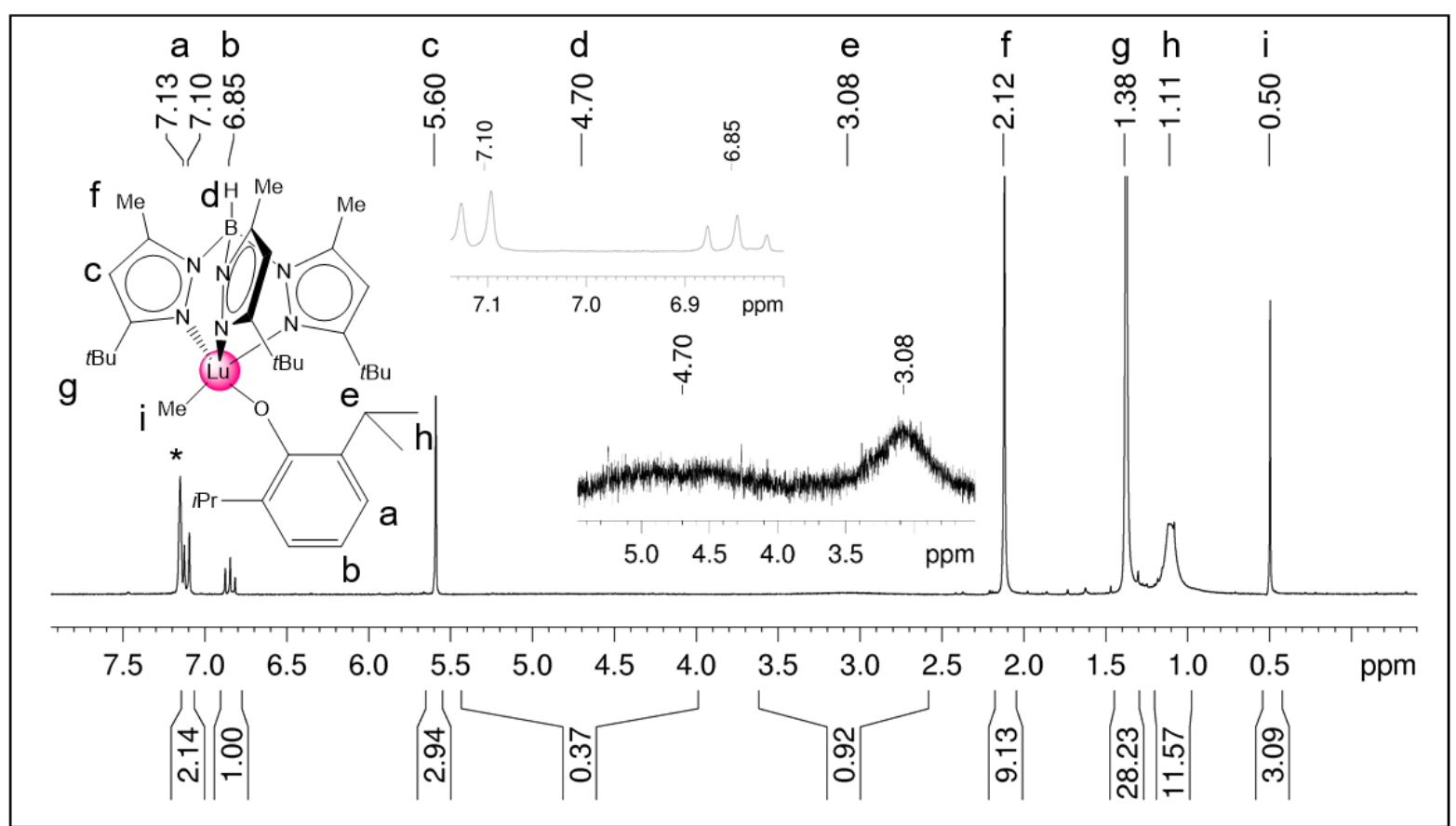

Figure S30. ${ }^{1} \mathrm{H}$ NMR spectrum (250 MHz, [D6]benzene) of complex Tp ${ }^{t B u, M e} L u M e\left(\mathrm{OC}_{6} \mathrm{H}_{3} / \mathrm{Pr}_{2}-2,6\right)(6-$ Lu) at $26^{\circ} \mathrm{C}$. 


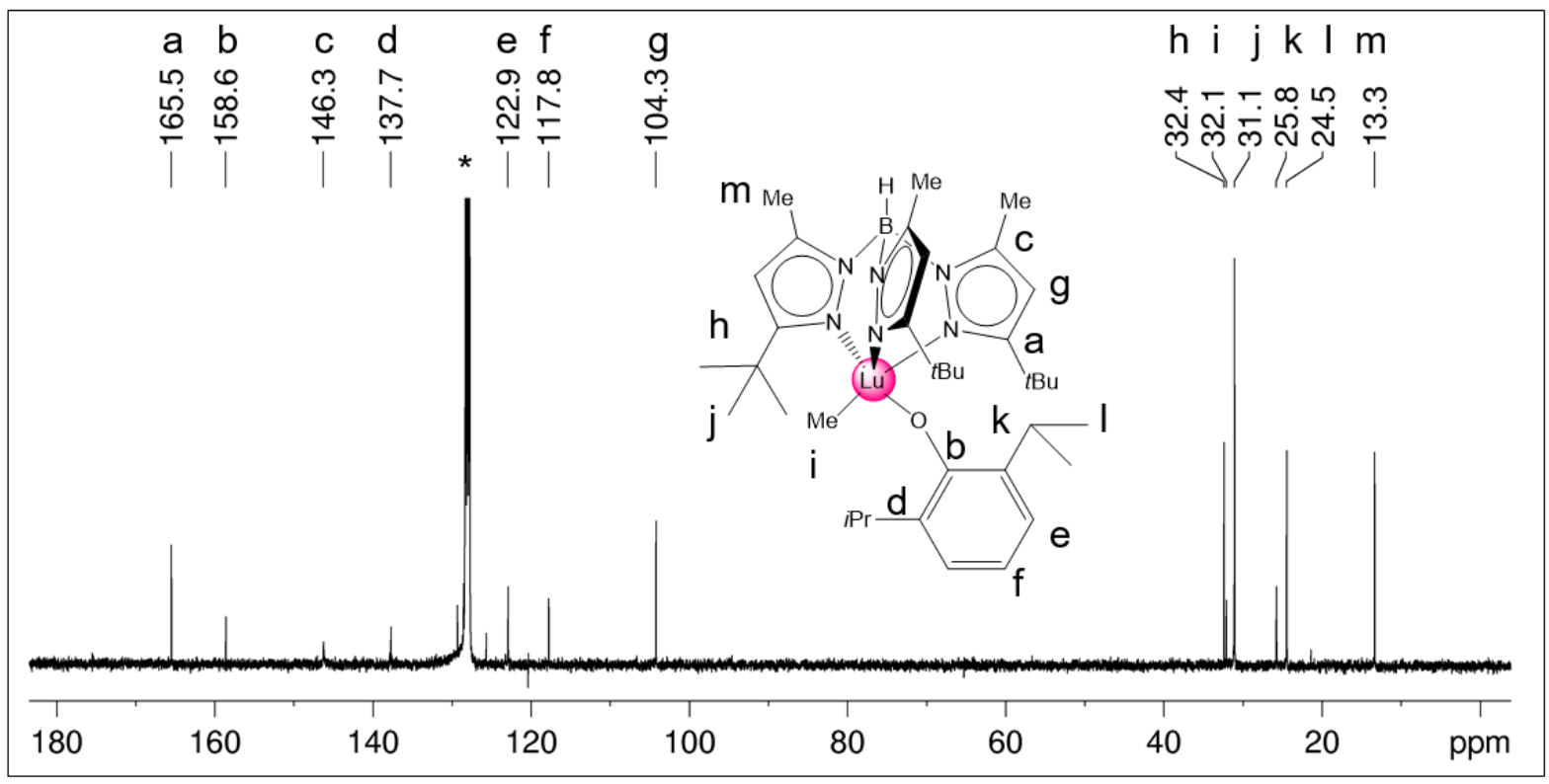

Figure S31. ${ }^{13} \mathrm{C}\left\{{ }^{1} \mathrm{H}\right\}$ NMR spectrum (63 MHz, [D6] benzene) of complex $\mathrm{Tp}^{\mathrm{tBu}, \mathrm{Me}} \mathrm{LuMe}\left(\mathrm{OC}_{6} \mathrm{H}_{3} / \mathrm{Pr}_{2}-2,6\right)$ (6-Lu) at $26^{\circ} \mathrm{C}$.

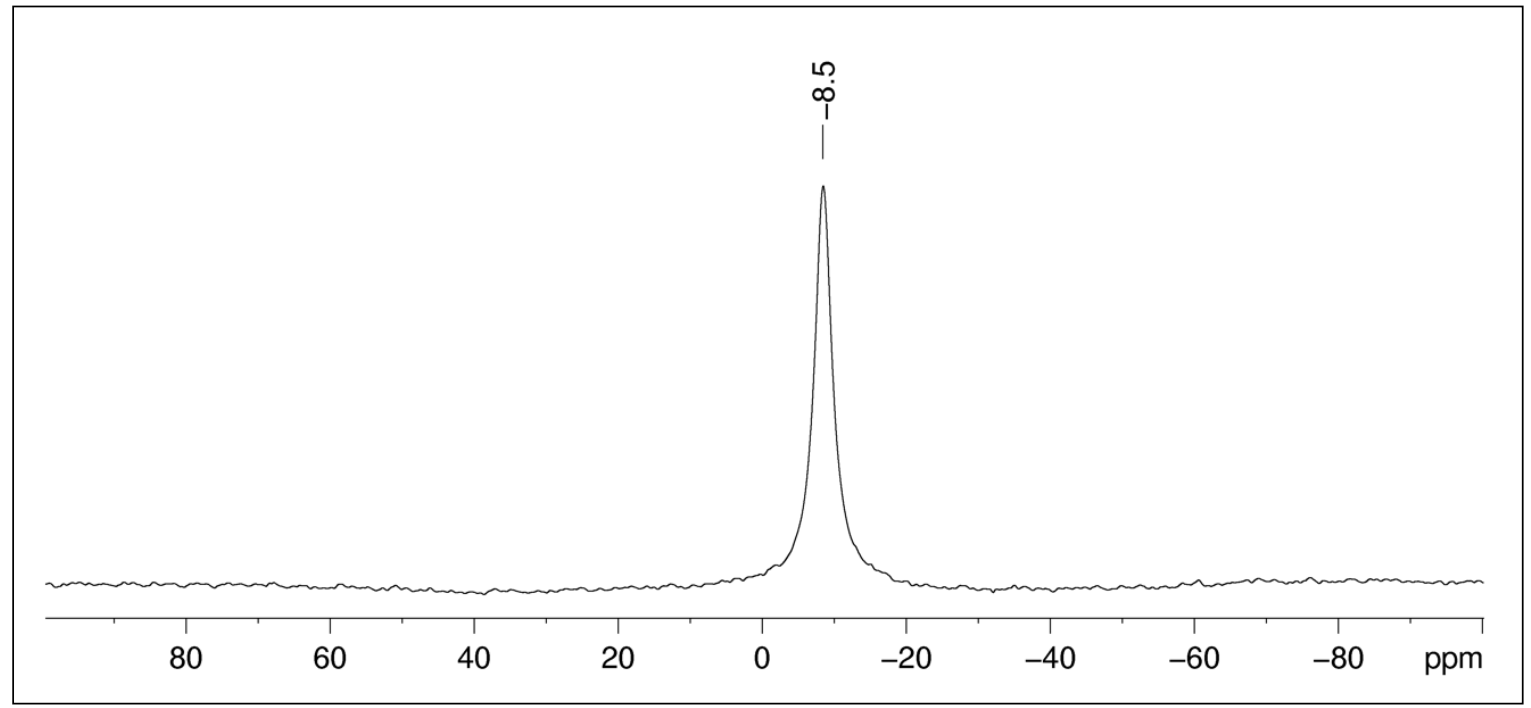

Figure S32. ${ }^{11} \mathrm{~B}\left\{{ }^{1} \mathrm{H}\right\}$ NMR spectrum (80 MHz, [D6]benzene) of complex $\mathrm{Tp}^{t \mathrm{Bu}, \mathrm{Me}} \mathrm{LuMe}\left(\mathrm{OC}_{6} \mathrm{H}_{3} / \mathrm{Pr}_{2}-2,6\right)(6-$ Lu) at $26^{\circ} \mathrm{C}$. 


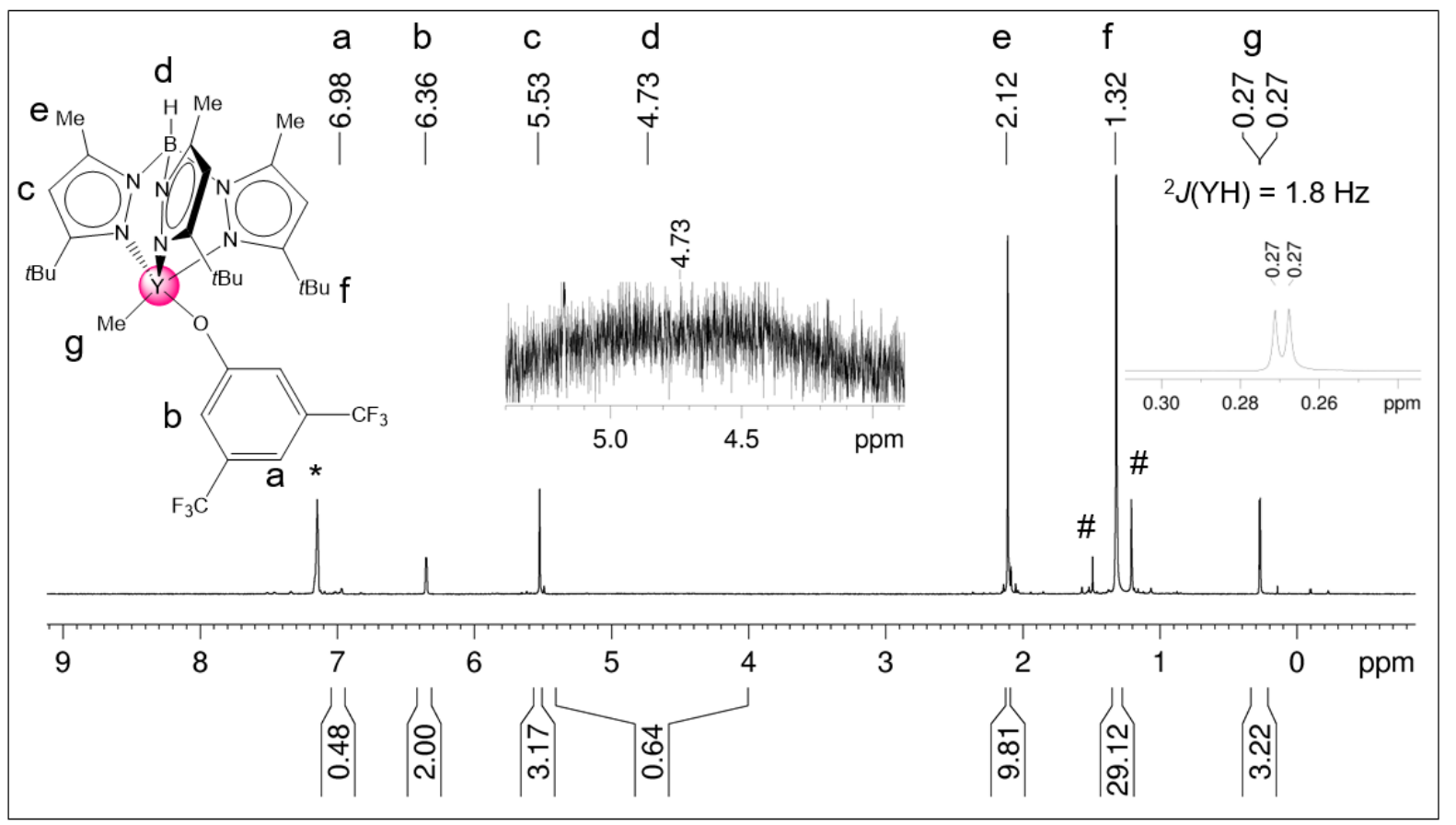

Figure S33. ${ }^{1} \mathrm{H}$ NMR spectrum $\left(250 \mathrm{MHz}\right.$, [D6]benzene) of complex $\mathrm{Tp}^{\mathrm{tBu}, \mathrm{Me}} \mathrm{YMe}_{6}\left[\mathrm{OC}_{6} \mathrm{H}_{3}\left(\mathrm{CF}_{3}\right)_{2}-3,5\right]$ (7Y) at $26{ }^{\circ} \mathrm{C}$ and traces of decomposition products (\#).

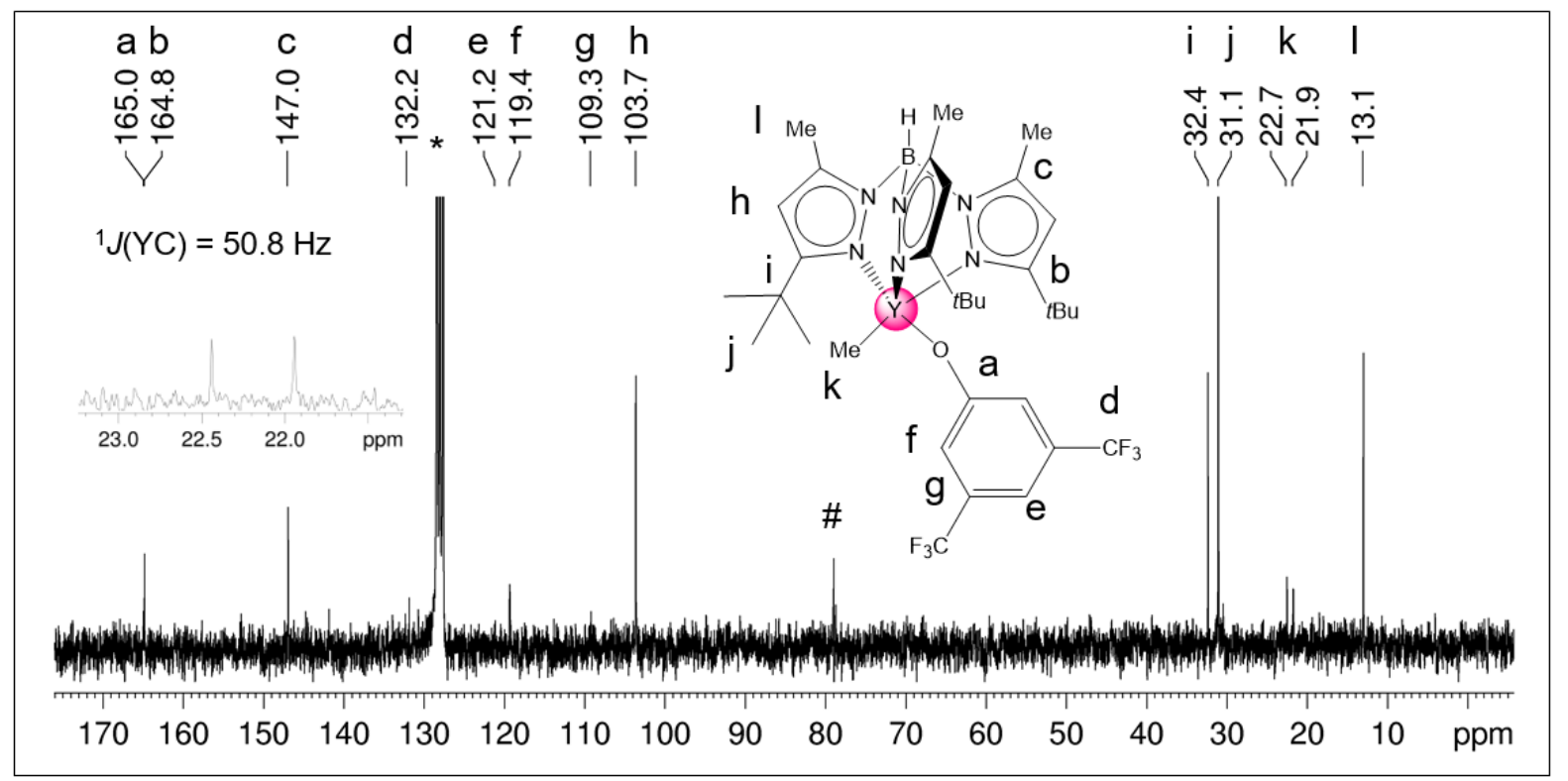

Figure S34. ${ }^{13} \mathrm{C}\left\{{ }^{1} \mathrm{H}\right\}$ NMR spectrum (63 MHz, [D $\left.\mathrm{D}_{6}\right]$ benzene) of complex $\mathrm{Tp}^{\mathrm{tBu}, \mathrm{Me}} \mathrm{YMe}\left[\mathrm{OC}_{6} \mathrm{H}_{3}\left(\mathrm{CF}_{3}\right)_{2}-3,5\right]$ $(7-\mathrm{Y})$ at $26^{\circ} \mathrm{C}$ and traces of decomposition products (\#). 


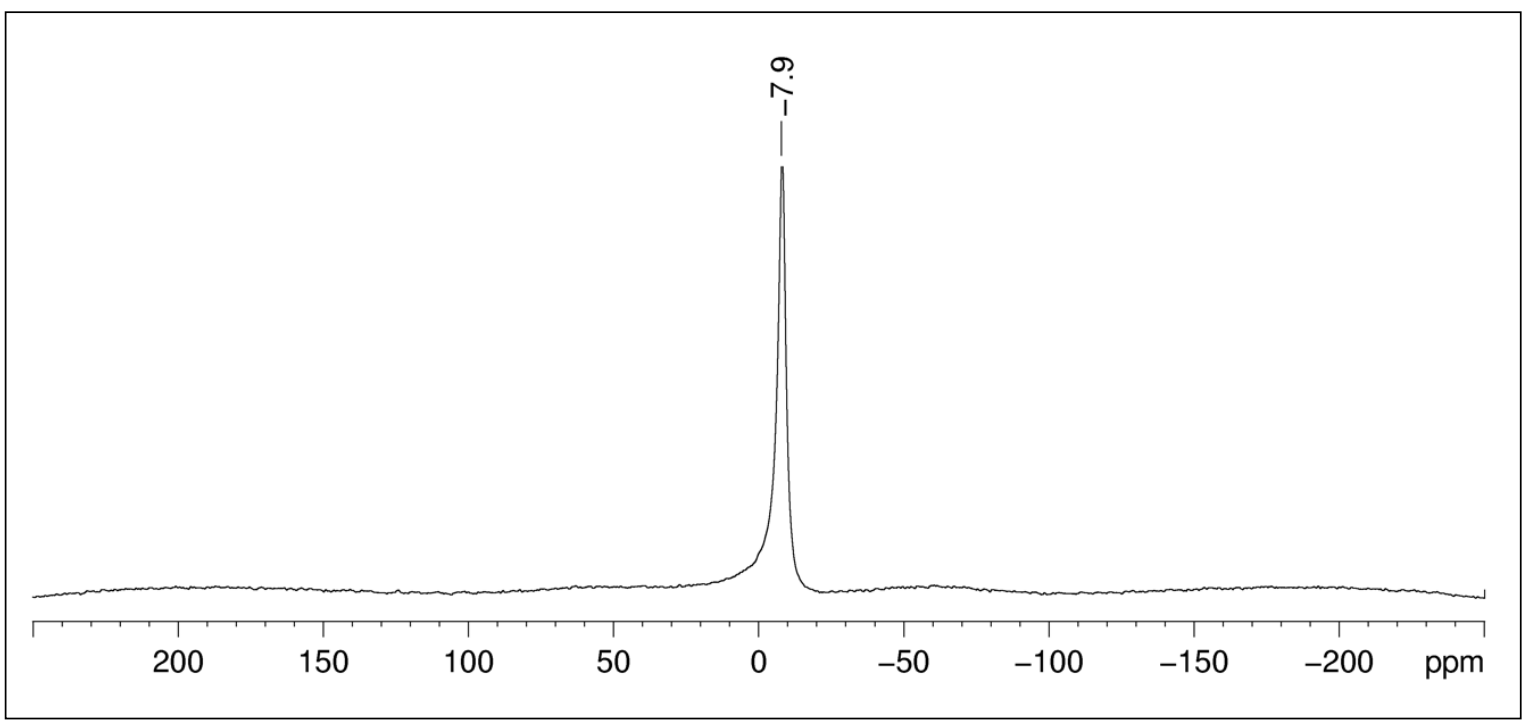

Figure S35. ${ }^{11} \mathrm{~B}\left\{{ }^{1} \mathrm{H}\right\} \mathrm{NMR}$ spectrum (80 MHz, [D $\left.\mathrm{D}_{6}\right]$ benzene) of complex $\mathrm{Tp}^{\mathrm{tBu}, \mathrm{Me}} \mathrm{YMe}\left[\mathrm{OC}_{6} \mathrm{H}_{3}\left(\mathrm{CF}_{3}\right)_{2}-3,5\right]$ 7-Y) at $26{ }^{\circ} \mathrm{C}$.

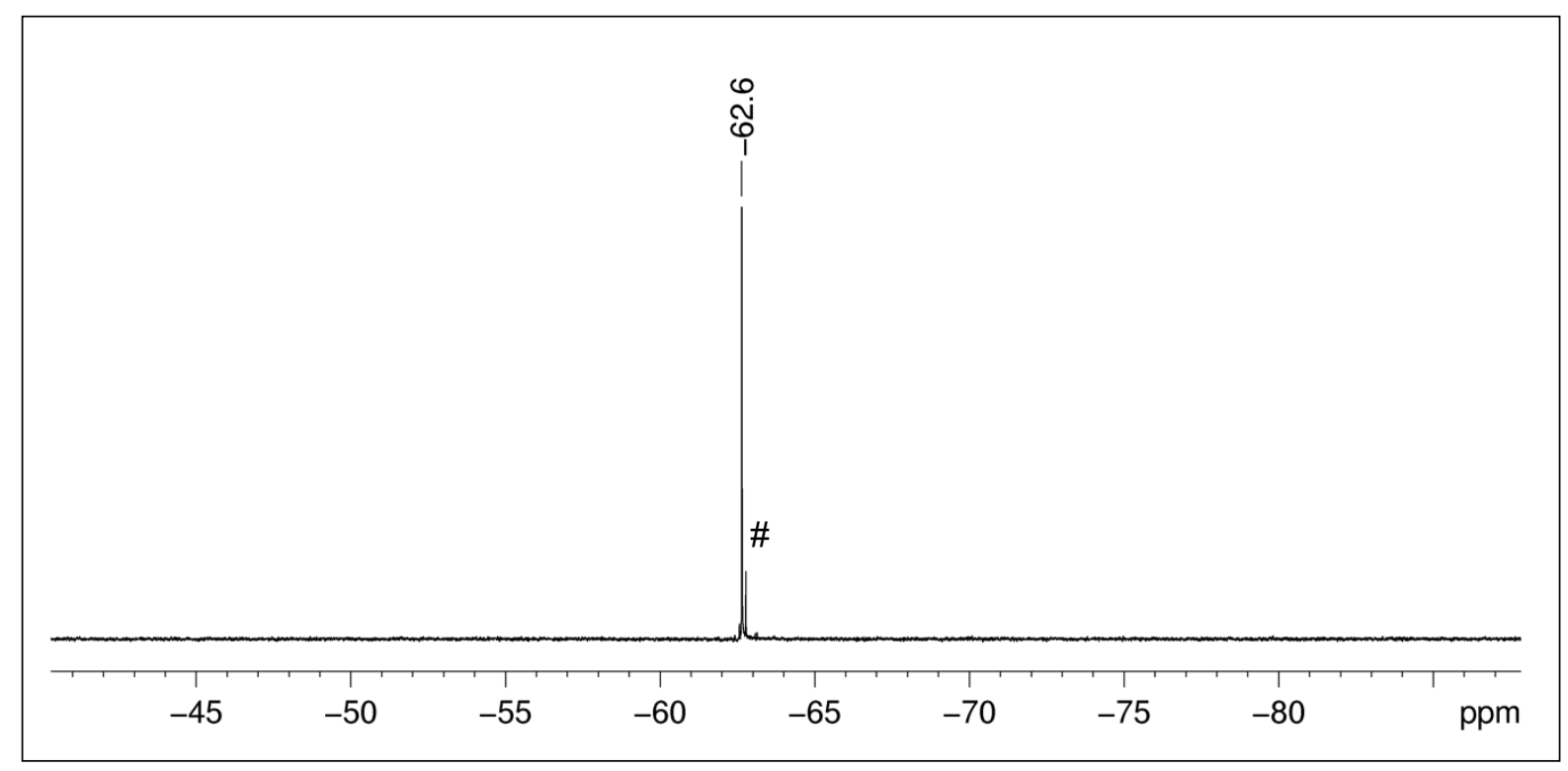

Figure S36. ${ }^{19} \mathrm{~F}\left\{{ }^{1} \mathrm{H}\right\}$ NMR spectrum (376 MHz, [D6]benzene) of complex $\operatorname{Tp}^{\mathrm{tBu}, \mathrm{Me}} \mathrm{YMe}\left[\mathrm{OC}_{6} \mathrm{H}_{3}\left(\mathrm{CF}_{3}\right)_{2}-3,5\right]$ (7-Y) at $26^{\circ} \mathrm{C}$ and traces of decomposition products (\#). 


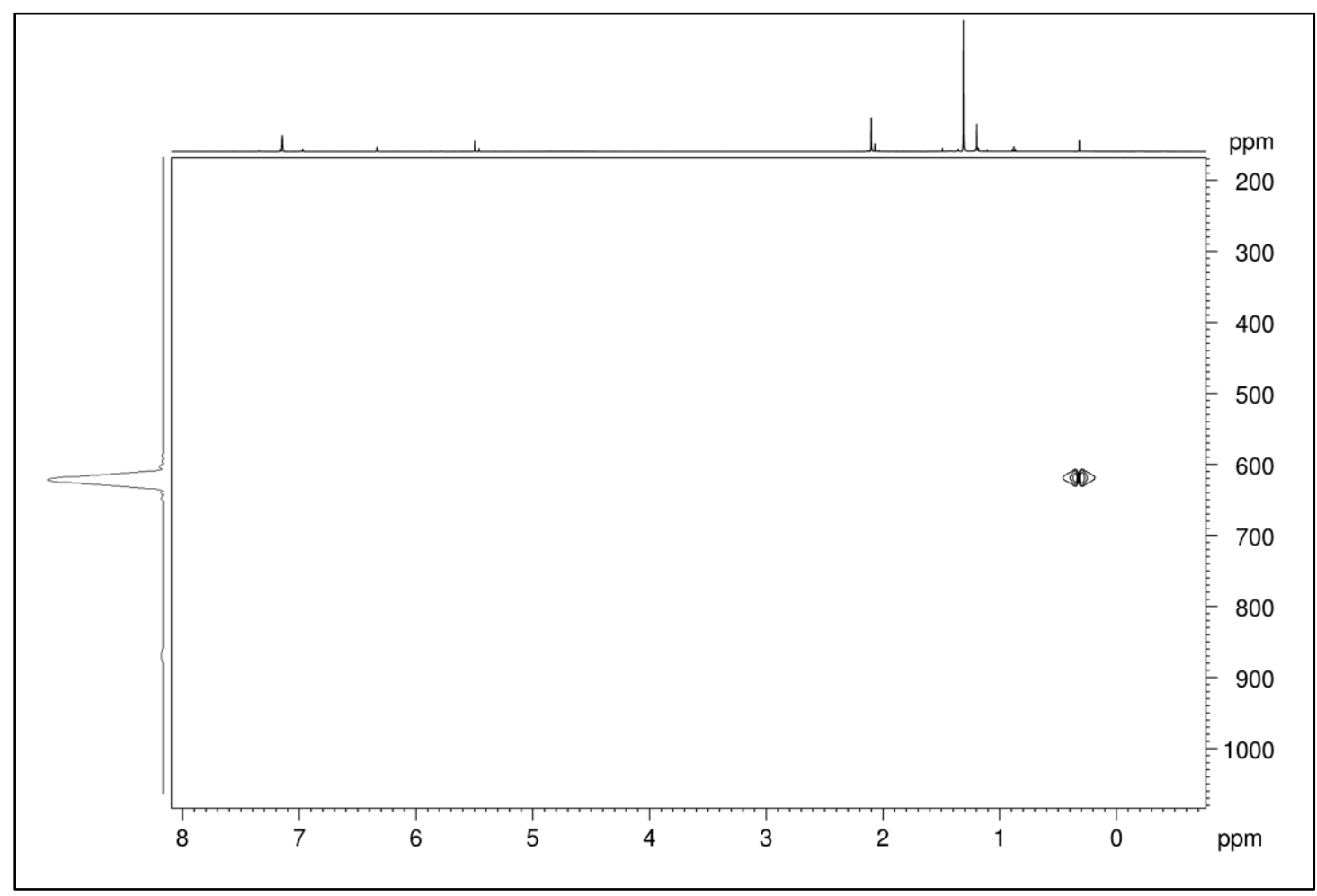

Figure S37. ${ }^{1} \mathrm{H}^{8}{ }^{89} \mathrm{Y} \mathrm{HSQC}$ NMR spectrum $\left(25 \mathrm{MHz}\right.$, [D $\left.\mathrm{D}_{6}\right]$ benzene $)$ of complex $\mathrm{Tp}{ }^{\mathrm{tBu}, \mathrm{Me}} \mathrm{YMe}\left[\mathrm{OC}_{6} \mathrm{H}_{3}\left(\mathrm{CF}_{3}\right)_{2^{-}}\right.$ $3,5](7-\mathrm{Y})$ at $26{ }^{\circ} \mathrm{C}$.

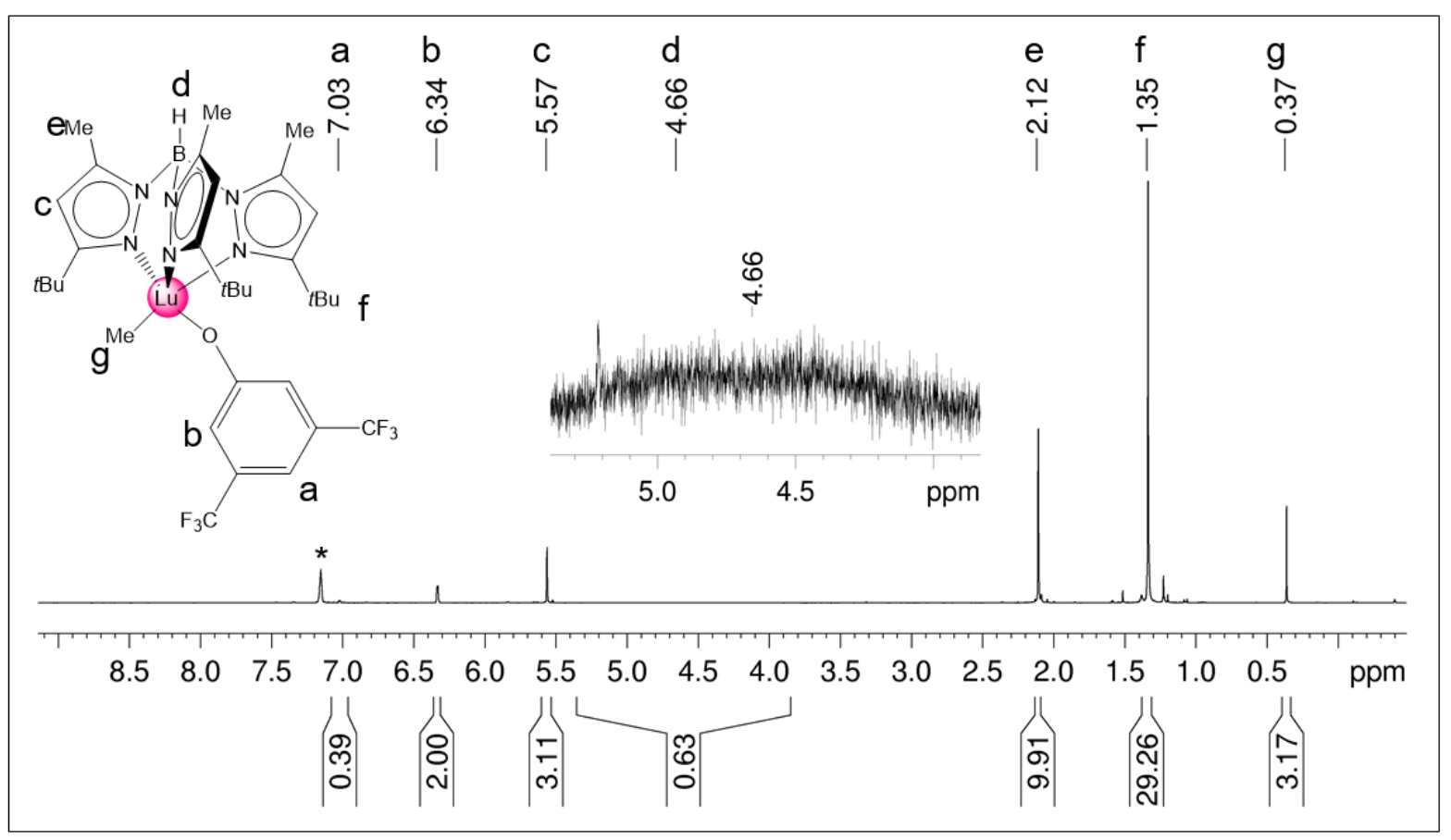

Figure S38. ${ }^{1} \mathrm{H}$ NMR spectrum $\left(250 \mathrm{MHz}\right.$, [D6]benzene) of complex $\mathrm{Tp}^{\mathrm{tB} u, \mathrm{Me}} \mathrm{LuMe}\left[\mathrm{OC}_{6} \mathrm{H}_{3}\left(\mathrm{CF}_{3}\right)_{2}-3,5\right](7-$ Lu) at $26^{\circ} \mathrm{C}$. 


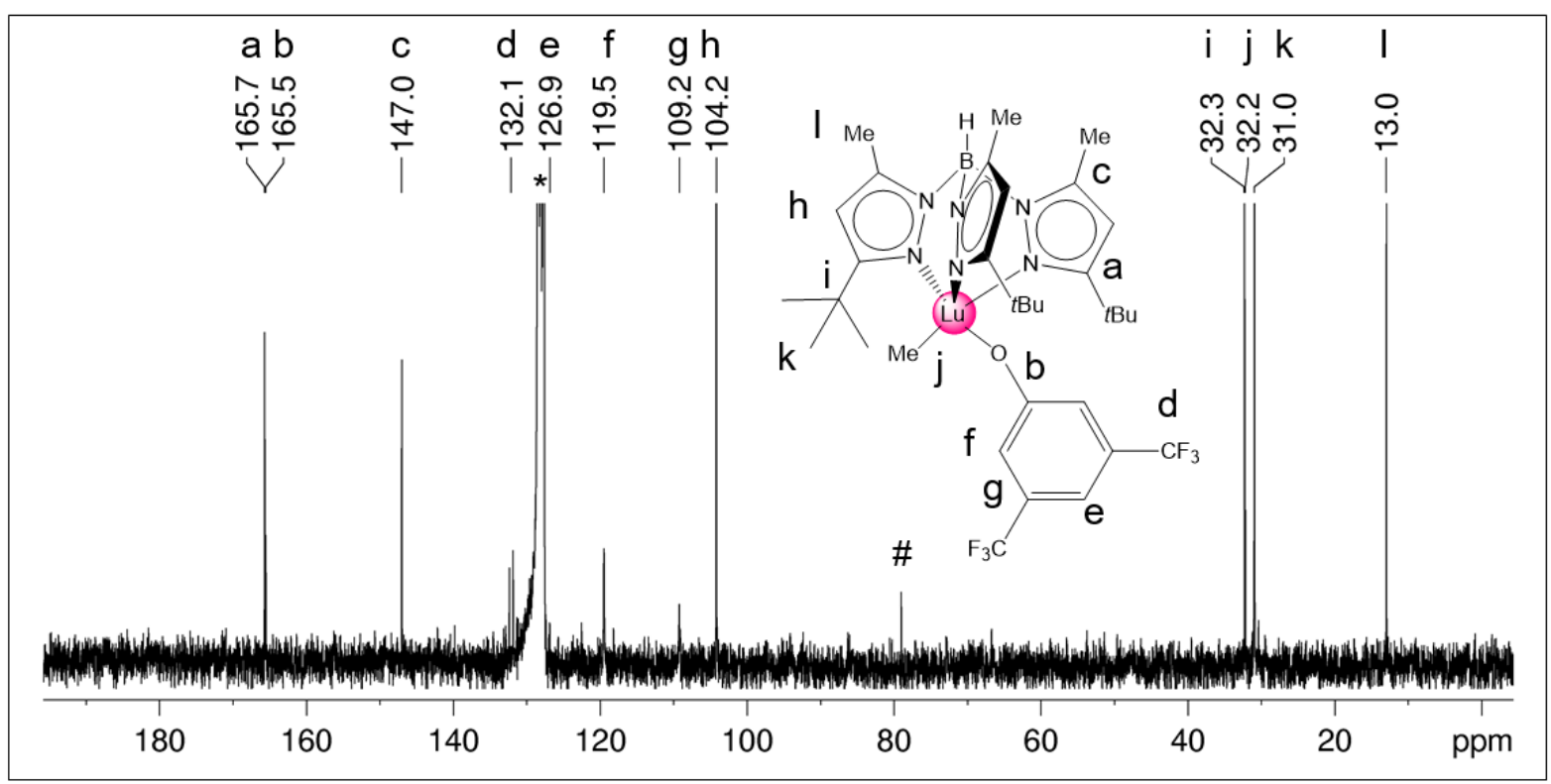

Figure S39. ${ }^{13} \mathrm{C}\left\{{ }^{1} \mathrm{H}\right\} \mathrm{NMR}$ spectrum (63 MHz, [D6]benzene) of complex Tp $\mathrm{D}^{\mathrm{tBu}, \mathrm{Me}} \mathrm{LuMe}\left[\mathrm{OC}_{6} \mathrm{H}_{3}\left(\mathrm{CF}_{3}\right)_{2}-3,5\right]$ (7-Lu) at $26^{\circ} \mathrm{C}$ and traces of decomposition products (\#).

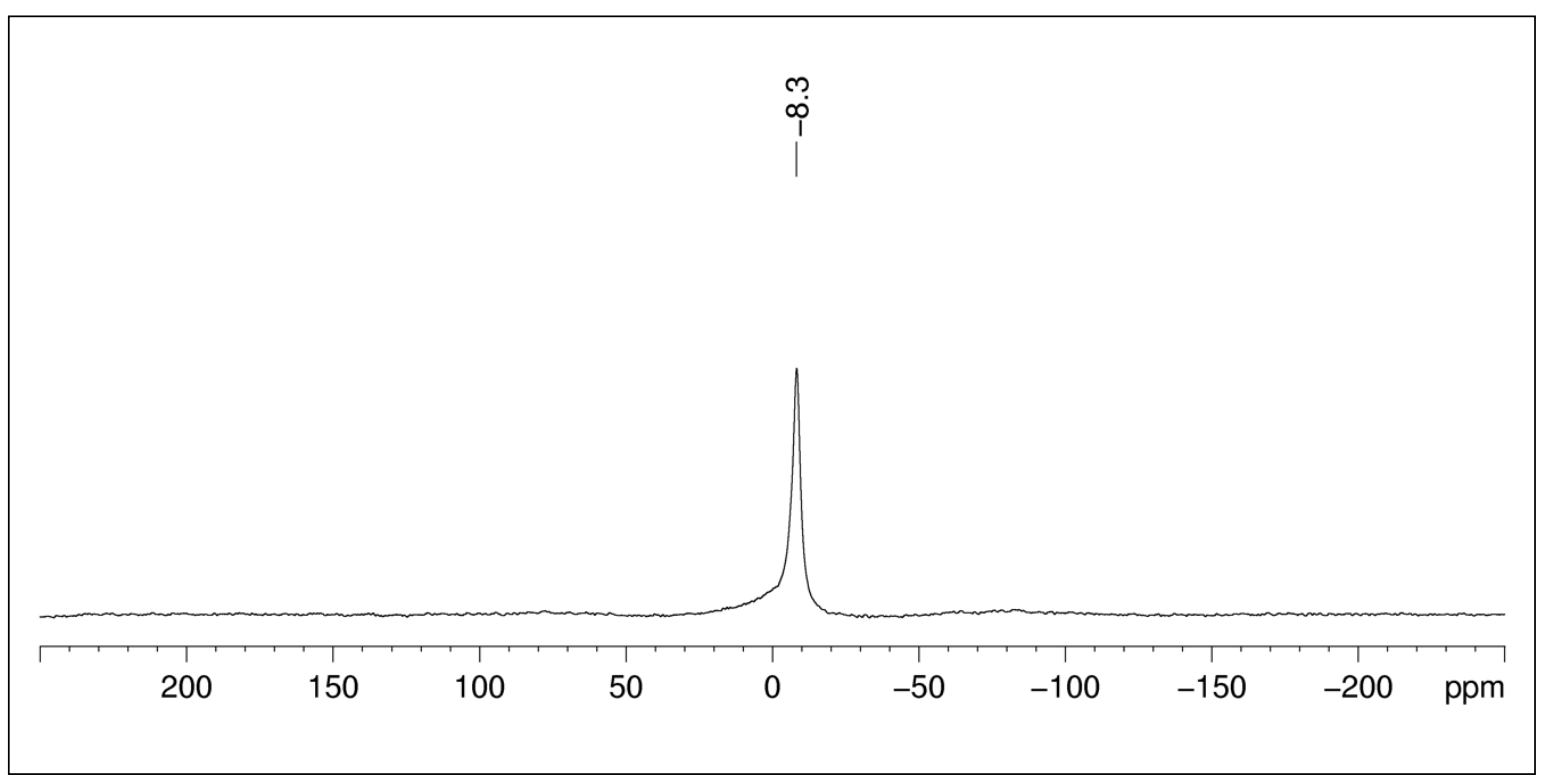

Figure S40. ${ }^{11} \mathrm{~B}\left\{{ }^{1} \mathrm{H}\right\} \mathrm{NMR}$ spectrum $\left(80 \mathrm{MHz},\left[\mathrm{D}_{6}\right]\right.$ benzene) of complex $\mathrm{Tp}^{\mathrm{tBu}, \mathrm{Me}} \mathrm{LuMe}\left[\mathrm{OC}_{6} \mathrm{H}_{3}\left(\mathrm{CF}_{3}\right)_{2}-3,5\right]$ (7-Lu) at $26{ }^{\circ} \mathrm{C}$. 


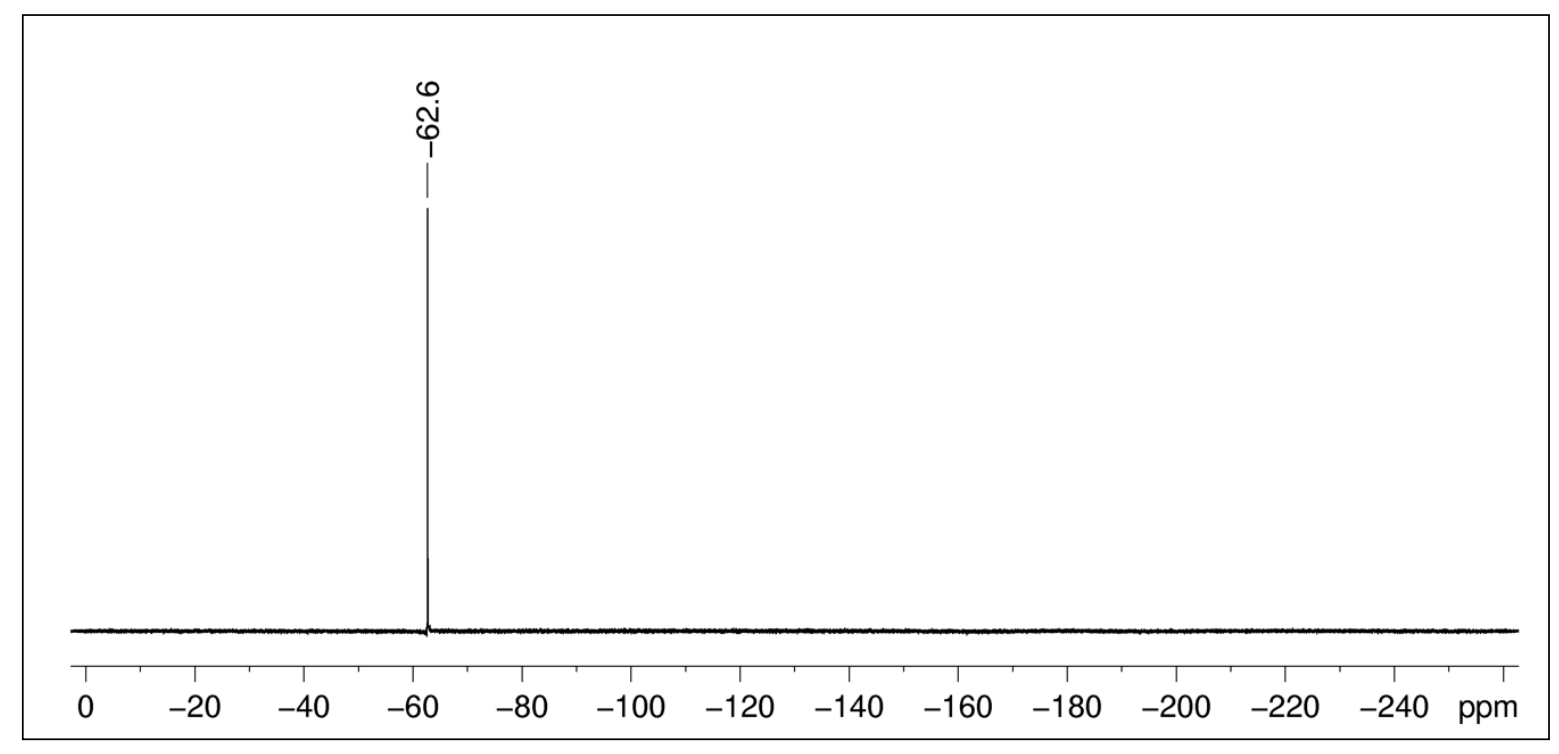

Figure S41. ${ }^{19} \mathrm{~F}\left\{{ }^{1} \mathrm{H}\right\}$ NMR spectrum (376 MHz, $\left[\mathrm{D}_{6}\right]$ benzene) of complex $\mathrm{Tp}^{\mathrm{tBu}, \mathrm{Me}} \mathrm{LuMe}\left[\mathrm{OC}_{6} \mathrm{H}_{3}\left(\mathrm{CF}_{3}\right)_{2}-\right.$ 3,5] (7-Lu) at $26^{\circ} \mathrm{C}$.

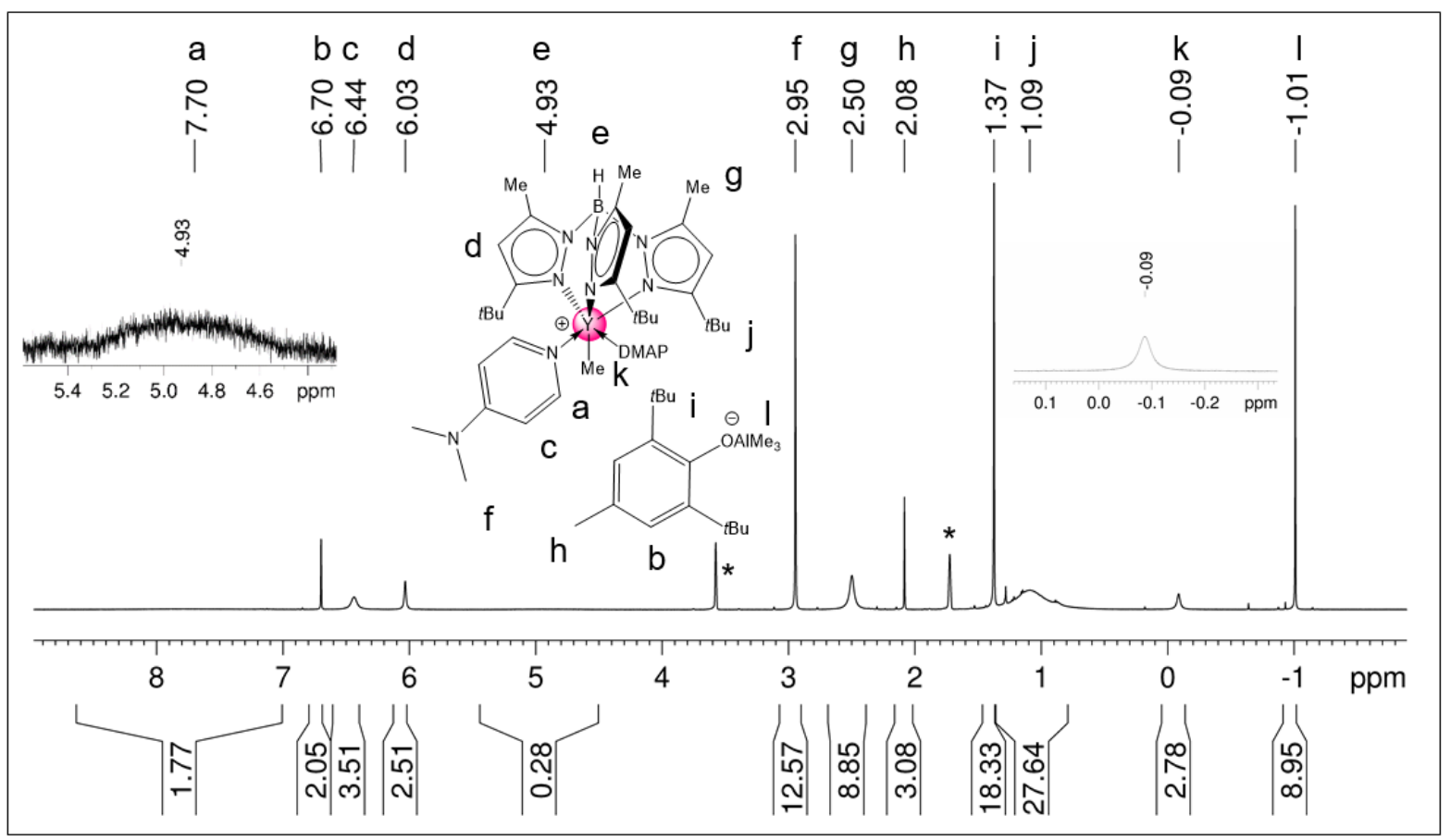

Figure S42. ${ }^{1} \mathrm{H}$ NMR spectrum $\left(500 \mathrm{MHz}\right.$, [D8]thf) of complex $\left.\left[\mathrm{Tp}^{\mathrm{tBu}, \mathrm{Me} Y M e(d m a p}\right)_{2}\right]\left[\mathrm{Me}_{3} \mathrm{Al}\left(\mathrm{OC}_{6} \mathrm{H}_{2} \mathrm{BBu}_{2}-\right.\right.$ 2,6-Me-4)] (8-Y) at $0{ }^{\circ} \mathrm{C}$, no $\mathrm{Y}-\mathrm{H}$ coupling was observed. 


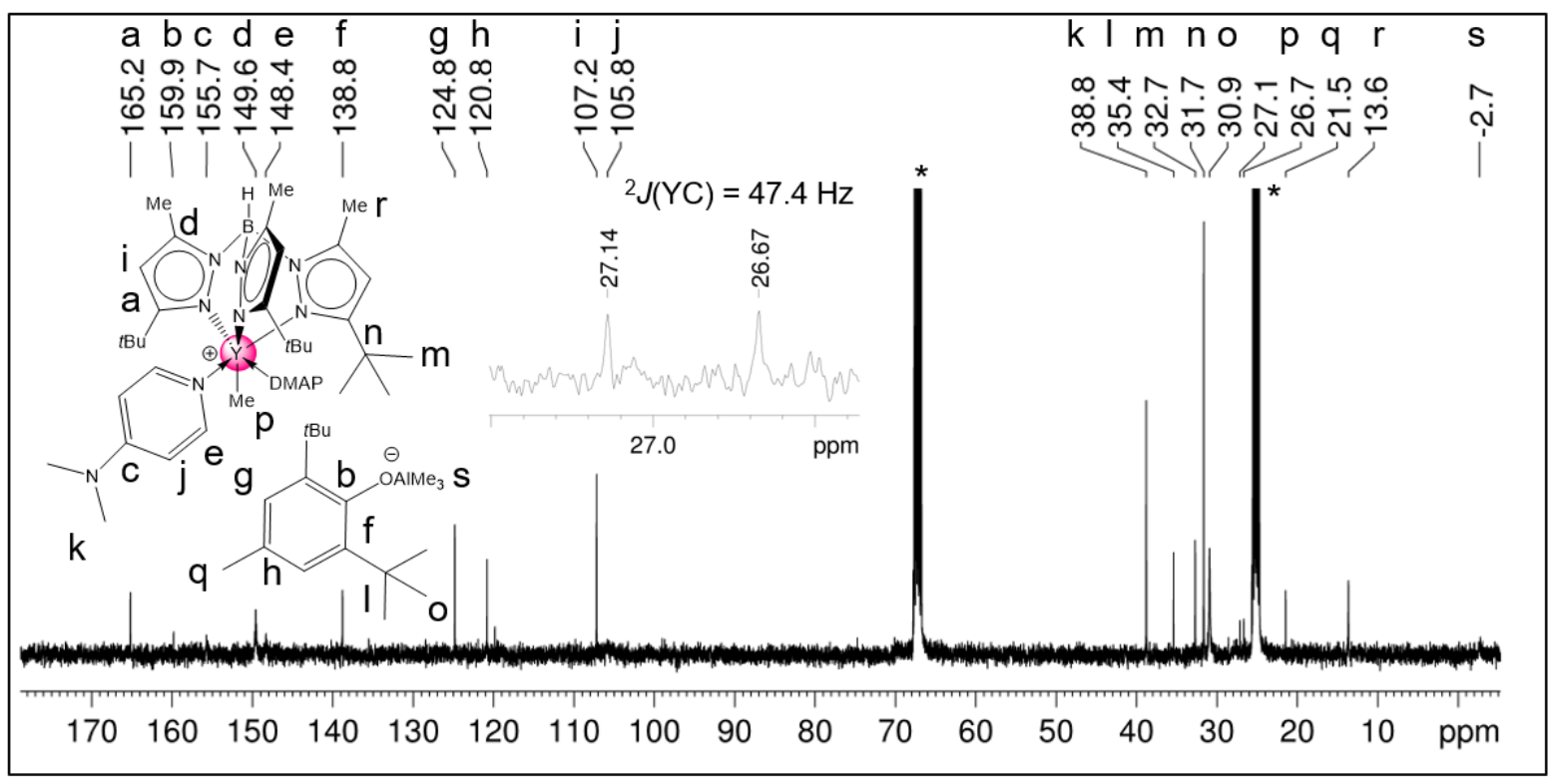

Figure S43. ${ }^{13} \mathrm{C}\left\{{ }^{1} \mathrm{H}\right\}$ NMR spectrum (101 MHz, [ $\left.\mathrm{D}_{8}\right]$ thf) of complex $\left[\mathrm{Tp}^{\mathrm{tBu}, \mathrm{Me}} \mathrm{YMe}(\mathrm{dmap})_{2}\right]\left[\mathrm{Me}_{3} \mathrm{Al}\left(\mathrm{OC}_{6} \mathrm{H}_{2} t \mathrm{Bu}_{2}-2,6-\mathrm{Me}-4\right)\right](8-\mathrm{Y})$ at $26^{\circ} \mathrm{C}$.

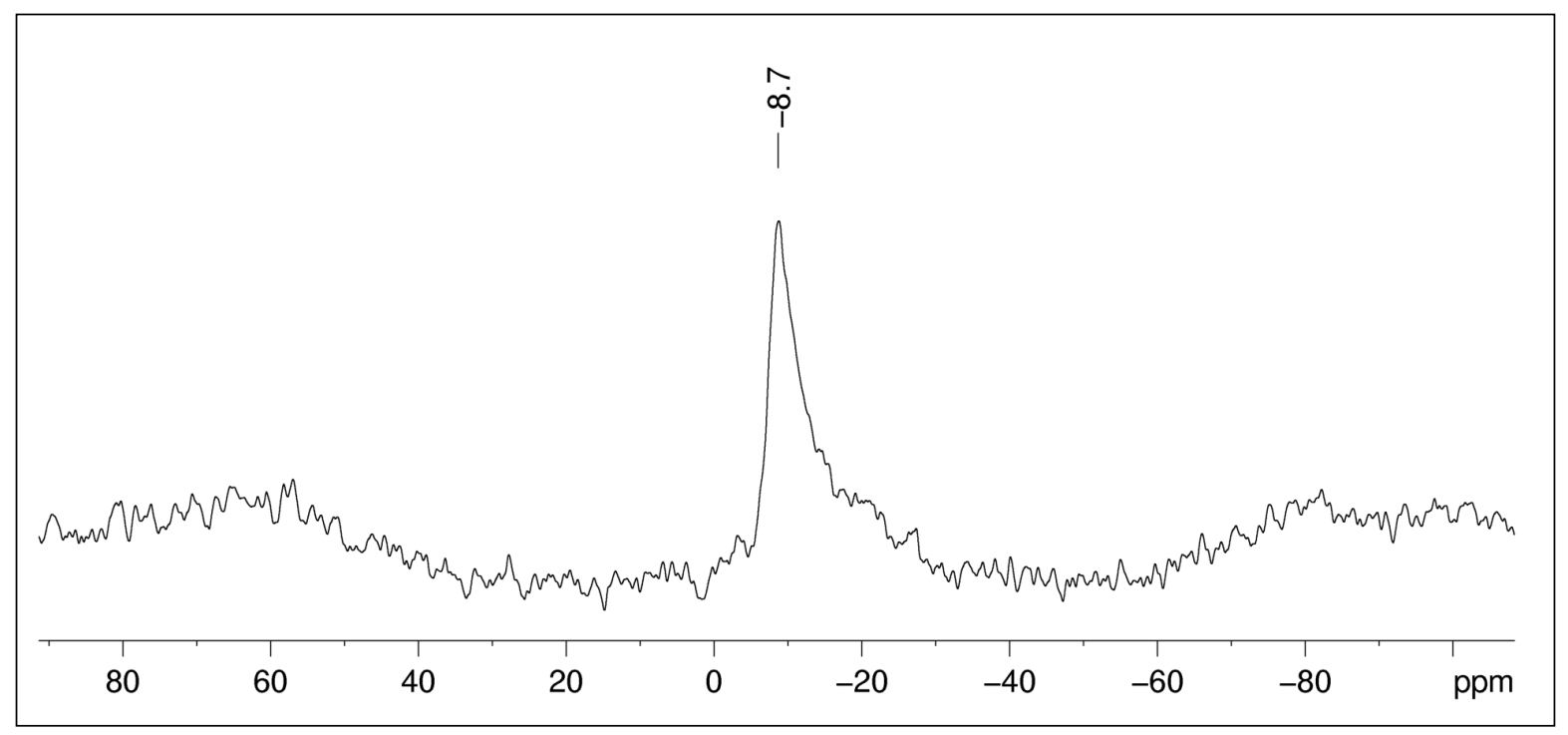

Figure S44. ${ }^{11} \mathrm{~B}\left\{{ }^{1} \mathrm{H}\right\}$ NMR spectrum $\left(80 \mathrm{MHz},\left[\mathrm{D}_{8}\right]\right.$ thf $)$ of complex $\left[\mathrm{Tp}^{t \mathrm{Bu}, \mathrm{Me}} \mathrm{YMe}(\mathrm{dmap})_{2}\right]\left[\mathrm{Me}_{3} \mathrm{Al}\left(\mathrm{OC}_{6} \mathrm{H}_{2} t \mathrm{Bu}_{2}-2,6-\mathrm{Me}-4\right)\right](8-\mathrm{Y})$ at $26^{\circ} \mathrm{C}$. 


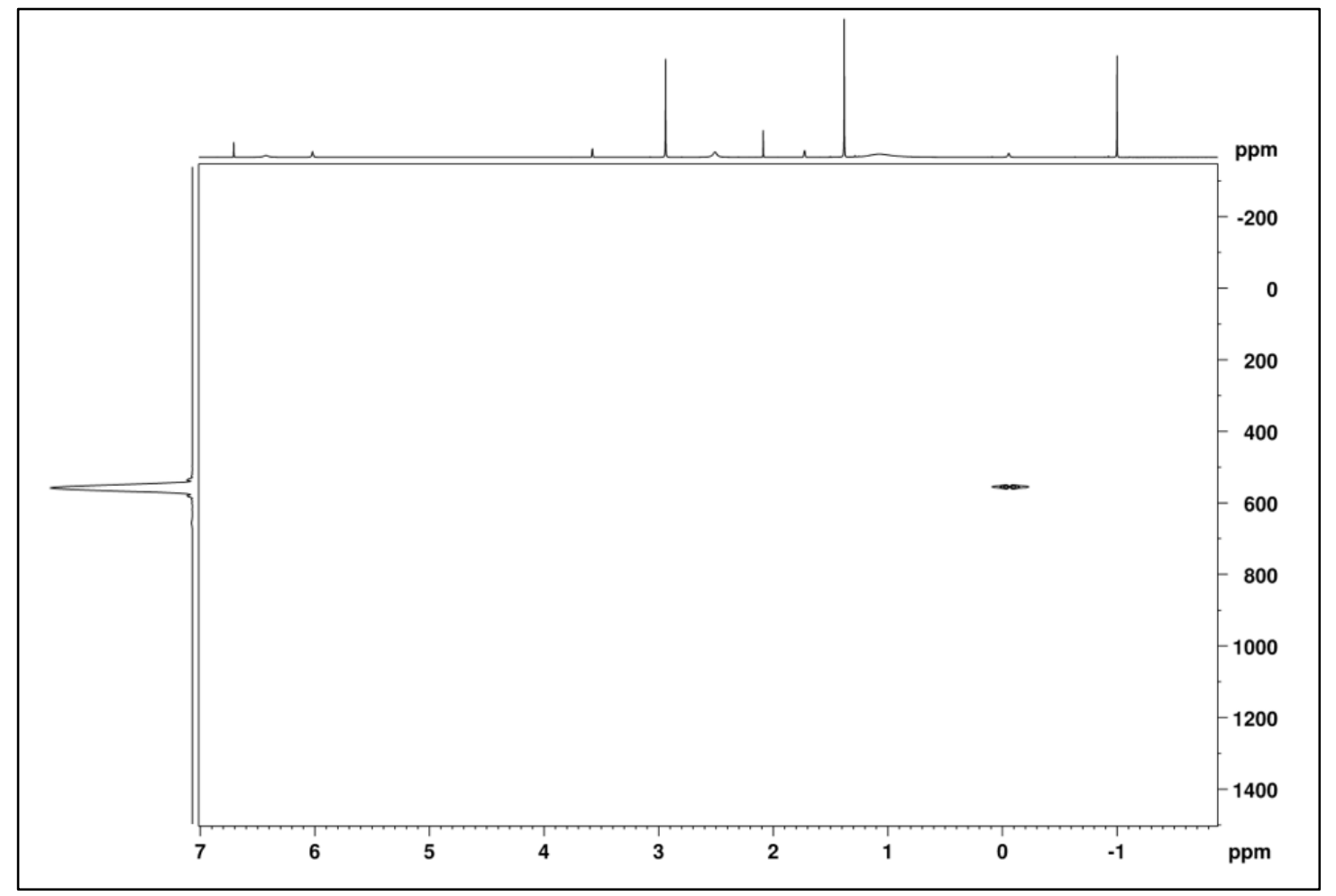

Figure S45. ${ }^{1} \mathrm{H}_{-}{ }^{89} \mathrm{Y}$ HSQC NMR spectrum $(25 \mathrm{MHz}$, [D6]thf) of complex $\left[\mathrm{Tp}^{\mathrm{tBu}, \mathrm{Me}} \mathrm{YMe}(\mathrm{dmap})_{2}\right]\left[\mathrm{Me}_{3} \mathrm{Al}\left(\mathrm{OC}_{6} \mathrm{H}_{2} \mathrm{BBu}_{2}-2,6-\mathrm{Me}-4\right)\right](8-\mathrm{Y})$ at $0{ }^{\circ} \mathrm{C}$.

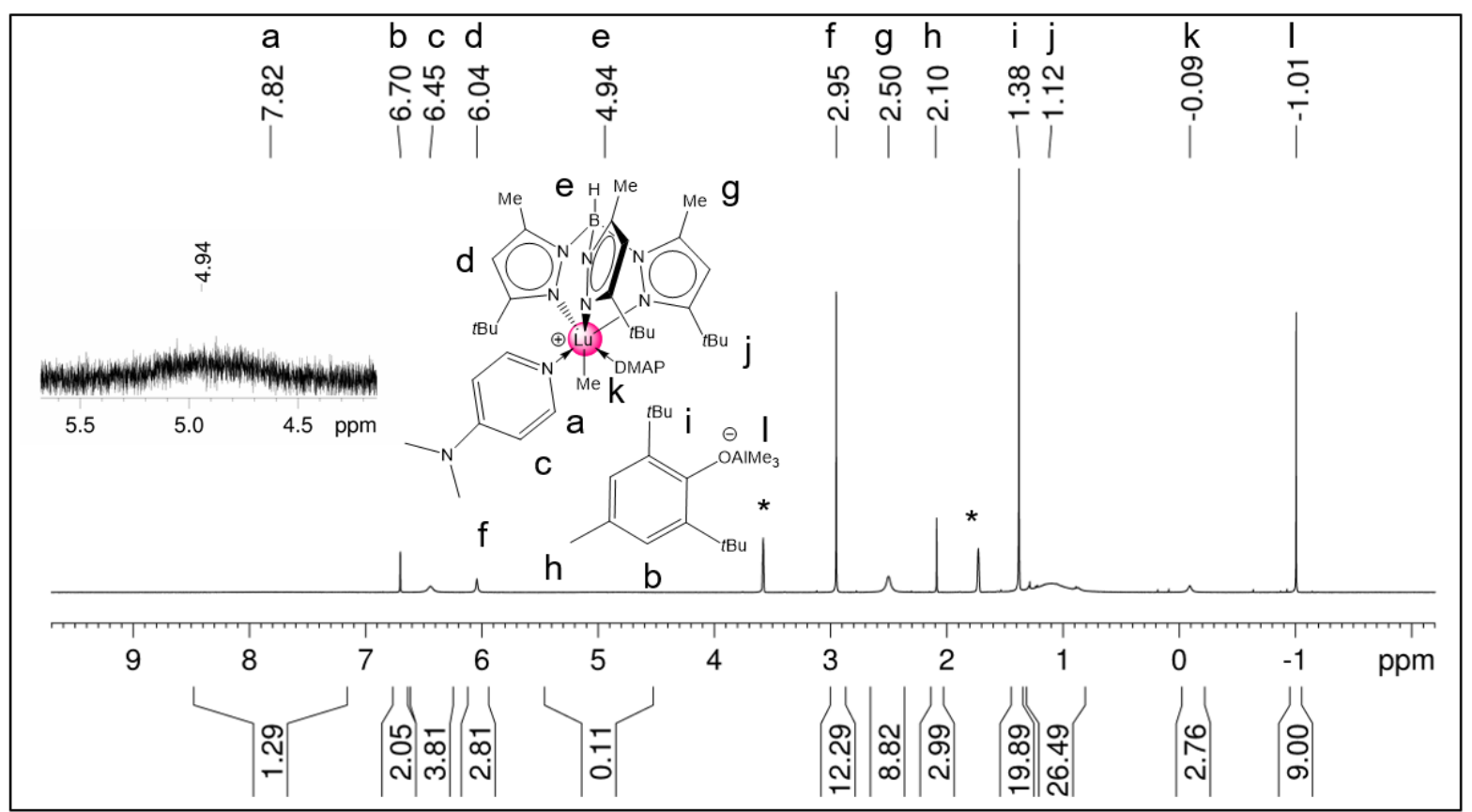

Figure S46. ${ }^{1} \mathrm{H}$ NMR spectrum $(500 \mathrm{MHz}$, [D8]thf) of complex

$\left[\mathrm{Tp}^{\mathrm{tBu}, \mathrm{Me}} \mathrm{LuMe}(\mathrm{dmap})_{2}\right]\left[\mathrm{Me}_{3} \mathrm{Al}\left(\mathrm{OC}_{6} \mathrm{H}_{2} t \mathrm{Bu} 2-2,6-\mathrm{Me}-4\right)\right](8-\mathrm{Lu})$ at $26{ }^{\circ} \mathrm{C}$. 


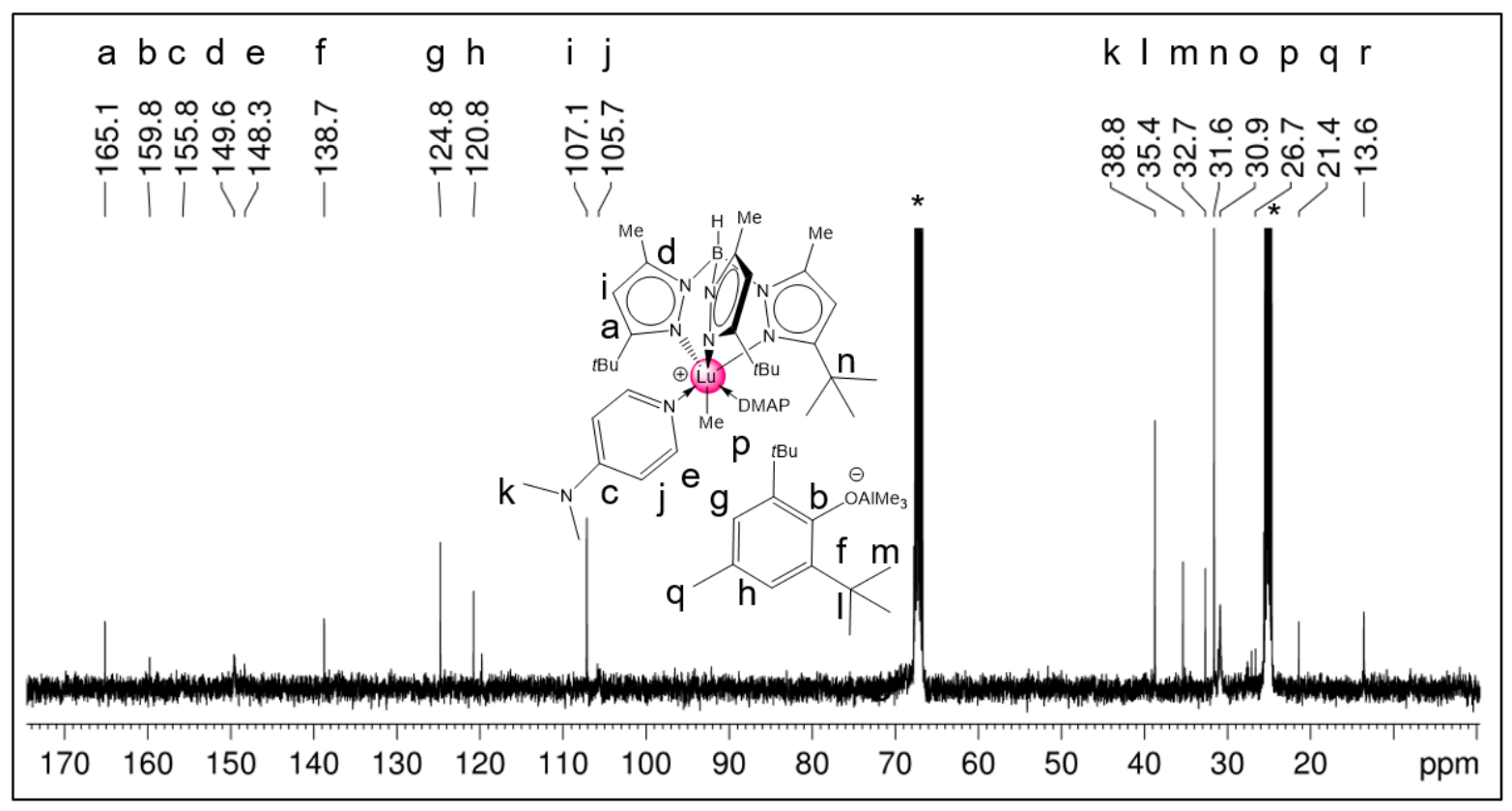

Figure S47. ${ }^{13} \mathrm{C}\left\{{ }^{1} \mathrm{H}\right\}$ NMR spectrum (101 MHz, [D8]thf) of complex $\left[\mathrm{Tp}^{t \mathrm{Bu}, \mathrm{Me}} \mathrm{LuMe}(\mathrm{dmap})_{2}\right]\left[\mathrm{Me}_{3} \mathrm{Al}\left(\mathrm{OC}_{6} \mathrm{H}_{2} t \mathrm{Bu}_{2}-2,6-\mathrm{Me}-4\right)\right](8-\mathrm{Lu})$ at $26{ }^{\circ} \mathrm{C}$.

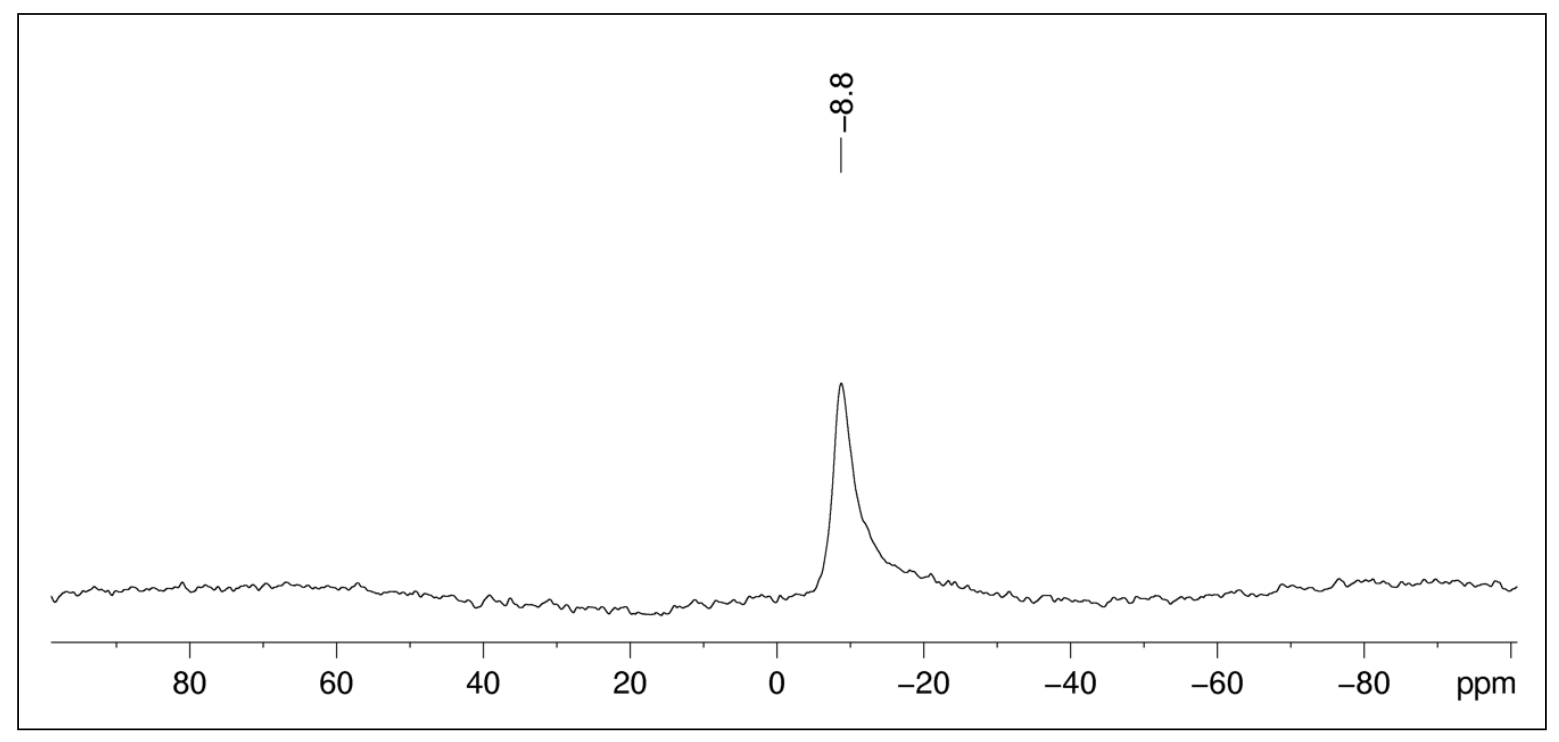

Figure S48. ${ }^{11} \mathrm{~B}\left\{{ }^{1} \mathrm{H}\right\} \mathrm{NMR}$ spectrum $\left(80 \mathrm{MHz},\left[\mathrm{D}_{8}\right]\right.$ thf $)$ of complex $\left[\mathrm{Tp}^{\mathrm{tBu}, \mathrm{Me}} \mathrm{LuMe}(\mathrm{dmap})_{2}\right]\left[\mathrm{Me}_{3} \mathrm{Al}\left(\mathrm{OC}_{6} \mathrm{H}_{2} t \mathrm{Bu}_{2}-2,6-\mathrm{Me}-4\right)\right](8-\mathrm{Lu})$ at $26^{\circ} \mathrm{C}$. 


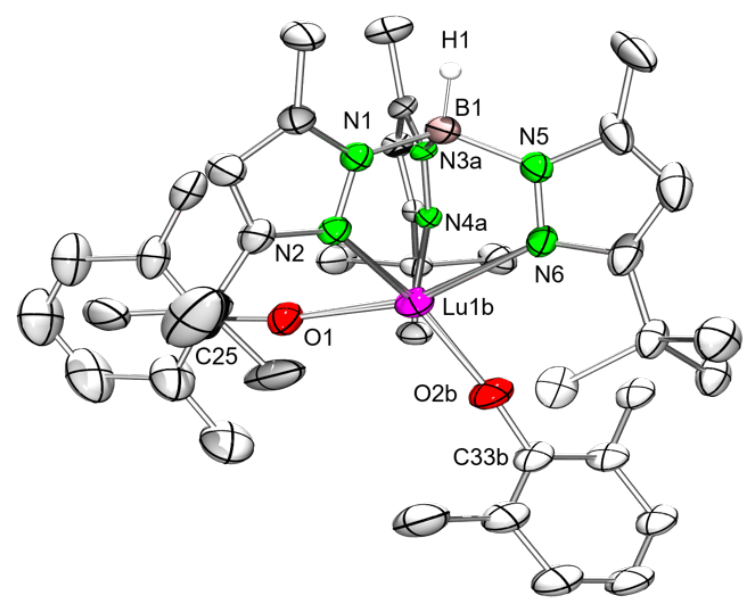

\begin{tabular}{|c|c|}
\hline 1-Lu & $\begin{array}{c}\text { Selected bond lengths } \\
\left.\text { and angles [A, }{ }^{\circ}\right]\end{array}$ \\
\hline Lu1b-N2 & $2.335(2)$ \\
\hline Lu1b-N4 & $2.362(9)$ \\
\hline Lu1b-N6 & $2.439(3)$ \\
\hline Lu1b-O1 & $2.052(2)$ \\
\hline Lu1b-O2b & $2.06(2)$ \\
\hline Lu1b-O1-C25 & $170.9(2)$ \\
\hline Lu1b-O2b-C33b & $174(3)$ \\
\hline
\end{tabular}

Figure S49. ORTEP representation of the crystal structure of 1-Lu with atomic displacement parameters set at the $50 \%$ level. Hydrogen atoms except for $\mathrm{BH}$ and the disorder in the tert-butyl groups, one aryloxy ligand, and one pyrazolyl ligand are omitted for clarity.

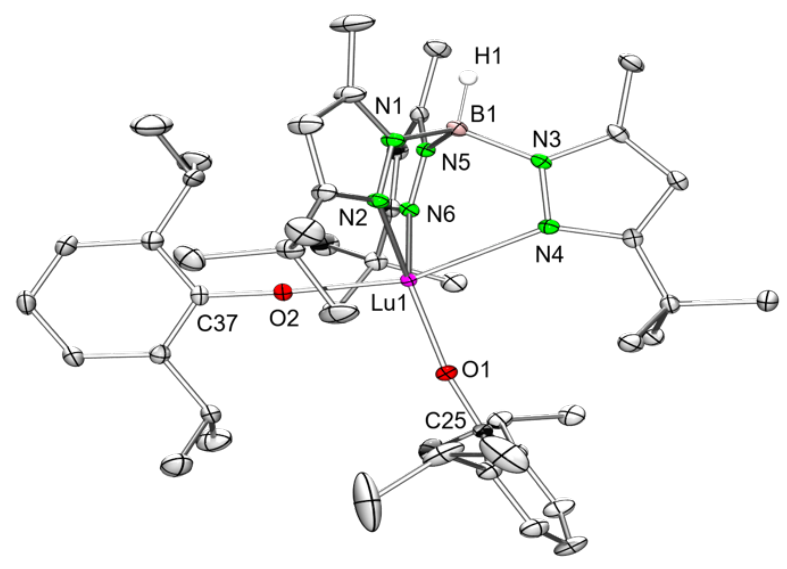

\begin{tabular}{|c|c|}
\hline 2-Lu & $\begin{array}{c}\text { Selected bond lengths } \\
\text { and angles [A, }{ }^{\circ} \text { ] }\end{array}$ \\
\hline Lu1-N2 & $2.365(1)$ \\
\hline Lu1-N4 & $2.517(1)$ \\
\hline Lu1-N6 & $2.370(1)$ \\
\hline Lu1-O1 & $2.072(1)$ \\
\hline Lu1-O2 & $2.038(1)$ \\
\hline Lu1-O1-C25 & $171.8(1)$ \\
\hline Lu1-O2-C37 & $175.4(1)$ \\
\hline
\end{tabular}

Figure S50. ORTEP representation of the crystal structure of 2-Lu with atomic displacement parameters set at the $50 \%$ level. Hydrogen atoms except for $\mathrm{BH}$, the second molecule in the unit cell and solvents are omitted for clarity.

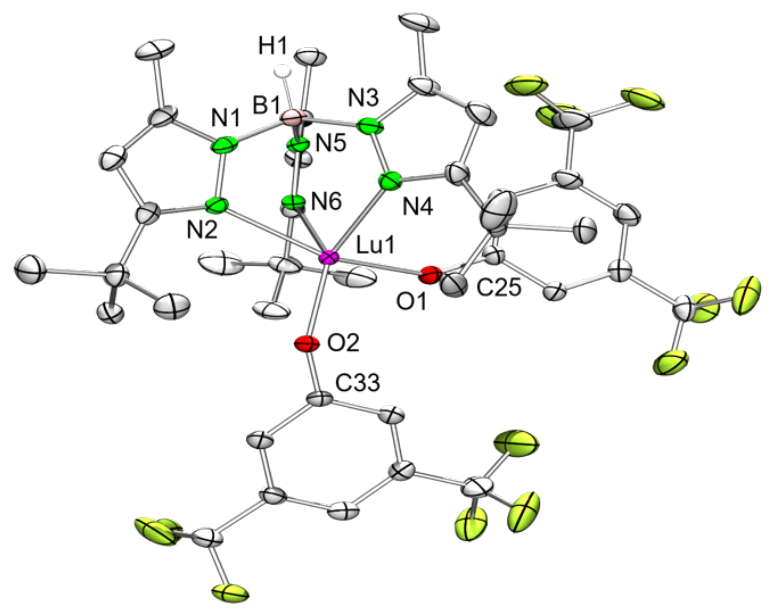

\begin{tabular}{|c|c|}
\hline 3-Lu & $\begin{array}{c}\text { Selected bond lengths } \\
\text { and angles [Aं, }{ }^{\circ} \text { ] }\end{array}$ \\
\hline Lu1-N2 & $2.397(2)$ \\
\hline Lu1-N4 & $2.318(2)$ \\
\hline Lu1-N6 & $2.315(2)$ \\
\hline Lu1-O1 & $2.090(2)$ \\
\hline Lu1-O2 & $2.064(2)$ \\
\hline Lu1-O1-C25 & $145.3(2)$ \\
\hline Lu1-O2-C33 & $147.3(2)$ \\
\hline
\end{tabular}

Figure S51. ORTEP representation of the crystal structure of 3-Lu with atomic displacement parameters set at the $50 \%$ level. Hydrogen atoms except for $\mathrm{BH}$ and the disorder in one $\mathrm{CF}_{3}$ group are omitted for clarity. 


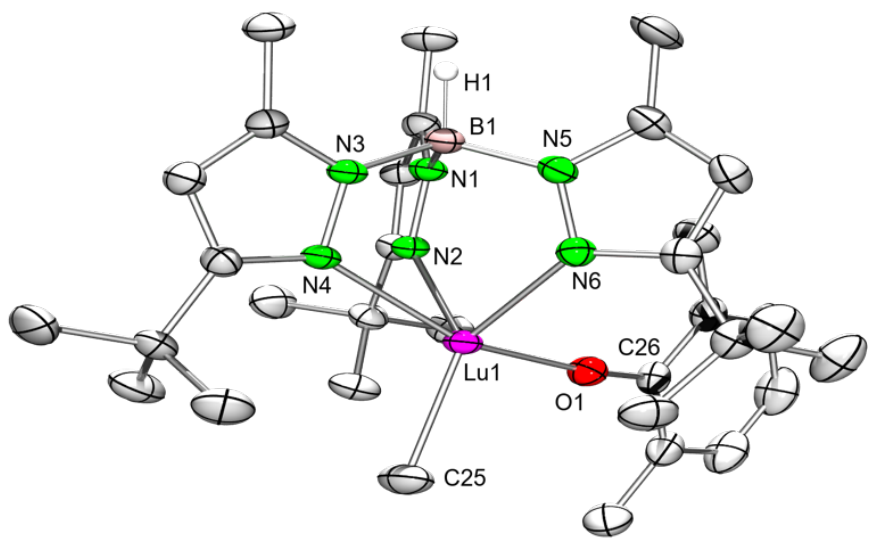

\begin{tabular}{|c|c|}
\hline 5-Lu & $\begin{array}{c}\text { Selected bond lengths } \\
\text { and angles [ } \mathbf{A}^{\circ}{ }^{\circ} \text { ] }\end{array}$ \\
\hline Lu1-N2 & $2.334(2)$ \\
\hline Lu1-N4 & $2.492(2)$ \\
\hline Lu1-N6 & $2.328(2)$ \\
\hline Lu1-O1 & $2.053(2)$ \\
\hline Lu1-C25 & $2.358(3)$ \\
\hline Lu1-O1-C26 & $167.2(2)$ \\
\hline
\end{tabular}

Figure S52. ORTEP representation of the crystal structure of 5-Lu with atomic displacement parameters set at the $50 \%$ level. Hydrogen atoms except for $\mathrm{BH}$ are omitted for clarity.

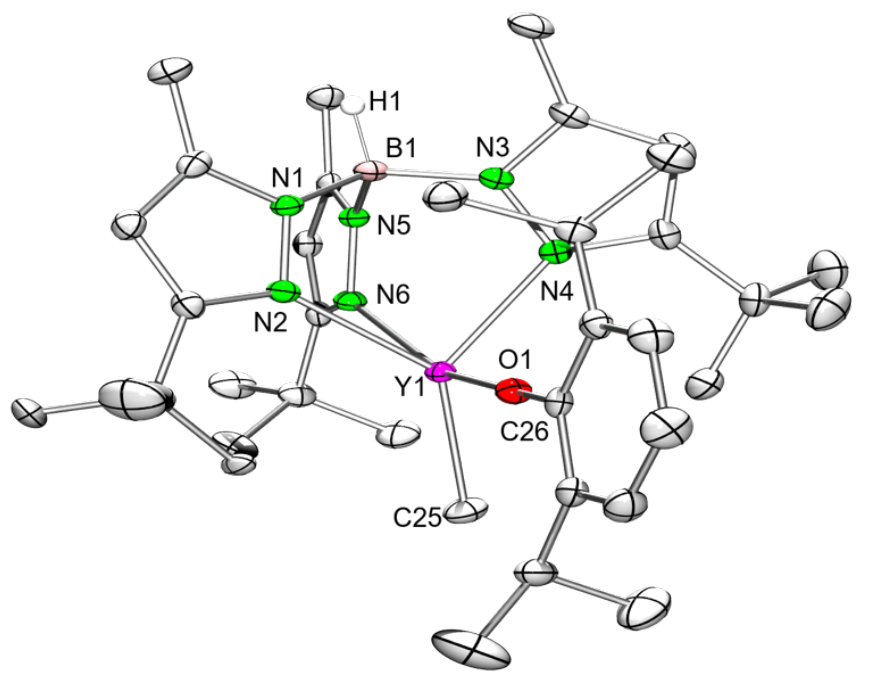

\begin{tabular}{|c|c|}
\hline $\mathbf{6 - Y}$ & $\begin{array}{c}\text { Selected bond lengths } \\
\text { and angles }\left[\AA \mathbf{A}^{\circ} \text { ] }\right.\end{array}$ \\
\hline $\mathrm{Y} 1-\mathrm{N} 2$ & $2.375(1)$ \\
\hline $\mathrm{Y} 1-\mathrm{N} 4$ & $2.384(2)$ \\
\hline $\mathrm{Y} 1-\mathrm{N} 6$ & $2.561(2)$ \\
\hline $\mathrm{Y} 1-\mathrm{O} 1$ & $2.087(1)$ \\
\hline $\mathrm{Y} 1-\mathrm{C} 25$ & $2.404(2)$ \\
\hline $\mathrm{Y} 1-\mathrm{O} 1-\mathrm{C} 26$ & $176.9(1)$ \\
\hline
\end{tabular}

Figure S53. ORTEP representation of the crystal structure of 6-Y with atomic displacement parameters set at the $50 \%$ level. Hydrogen atoms except for $\mathrm{BH}$, the disorder in one tert-butyl group and solvent molecules are omitted for clarity.

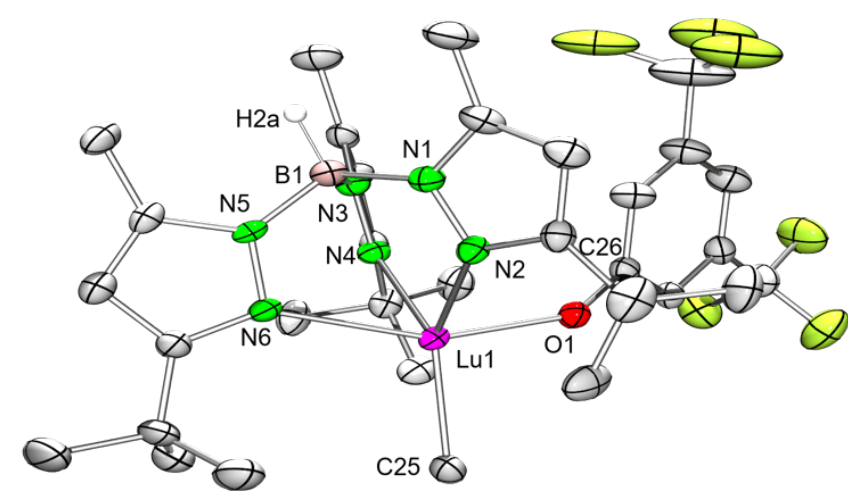

\begin{tabular}{|c|c|}
\hline 7-Lu & $\begin{array}{l}\text { Selected bond lengths } \\
\text { and angles }\left[\AA,{ }^{\circ}\right]\end{array}$ \\
\hline Lu1-N2 & $2.319(4)$ \\
\hline Lu1-N4 & $2.322(4)$ \\
\hline Lu1-N6 & $2.442(4)$ \\
\hline Lu1-O1 & $2.085(3)$ \\
\hline Lu1-C25 & $2.339(6)$ \\
\hline Lu1-O1-C26 & $150.9(3)$ \\
\hline
\end{tabular}

Figure S54. ORTEP representation of the crystal structure of 7-Lu with atomic displacement parameters set at the $50 \%$ level. Hydrogen atoms except for $\mathrm{BH}$, the second molecule in the unit cell and the disorder in one $\mathrm{CF}_{3}$ group are omitted for clarity. 
Table S1. Comprehensive crystallographic data for compounds 1-Lu, 2-Lu, 3-Lu, and 4-Lu

\begin{tabular}{|c|c|c|c|c|}
\hline & 1-Lu & 2-Lu & 3-Lu & 4-Lu \\
\hline CCDC & 1952866 & 1952862 & 1952859 & 1952861 \\
\hline formula & $\mathrm{C}_{40} \mathrm{H}_{58} \mathrm{BLuN}_{6} \mathrm{O}_{2}$ & $\begin{array}{l}\mathrm{C}_{48} \mathrm{H}_{74} \mathrm{BLuN}_{6} \mathrm{O}_{2} \bullet \\
1 / 2 \mathrm{C}_{7} \mathrm{H}_{8}\end{array}$ & $\mathrm{C}_{40} \mathrm{H}_{46} \mathrm{BF}_{12} \mathrm{LuN}_{6} \mathrm{O}_{2}$ & $\mathrm{C}_{40} \mathrm{H}_{66} \mathrm{BLuN}_{6} \mathrm{O}$ \\
\hline $\mathrm{M}\left[\mathrm{g} \mathrm{mol}^{-1}\right]$ & 839.69 & 998.97 & 1056.61 & 832.76 \\
\hline crystal system & triclinic & triclinic & monoclinic & monoclinic \\
\hline space group & $P \overline{1}$ & $P \overline{1}$ & $\mathrm{P} \mathrm{2} / \mathrm{c}$ & $\mathrm{P} \mathrm{2}{ }_{1} / \mathrm{c}$ \\
\hline$a[\AA]$ & $13.2578(12)$ & $10.0205(6)$ & $11.2564(8)$ & $19.301(2)$ \\
\hline $\mathrm{b}[\AA]$ & $14.1925(13)$ & $22.1089(14)$ & $21.3657(14)$ & $9.9326(11)$ \\
\hline$c[\AA]$ & $14.9775(14)$ & $23.8154(15)$ & $18.8036(13)$ & $22.217(2)$ \\
\hline$\alpha\left[^{\circ}\right]$ & $90.420(2)$ & $76.770(2)$ & 90 & 90 \\
\hline$\beta\left[^{\circ}\right]$ & $109.7010(10)$ & $78.528(2)$ & $96.4670(10)$ & $104.378(2)$ \\
\hline $\mathrm{Y}\left[\left[^{\circ}\right]\right.$ & $103.9910(10)$ & $84.220(2)$ & 90 & 90 \\
\hline $\mathrm{V}\left[\AA^{3}\right]$ & $2562.3(4)$ & $5025.0(5)$ & $4493.5(5)$ & $4125.8(8)$ \\
\hline Z & 2 & 4 & 4 & 4 \\
\hline $\mathrm{T}[\mathrm{K}]$ & 102(2) & $100(2)$ & $100(2)$ & $100(2)$ \\
\hline$\rho_{\text {calcd }}\left[\mathrm{g} \mathrm{cm}^{-3}\right]$ & 1.090 & 1.320 & 1.562 & 1.341 \\
\hline$\mu\left[\mathrm{mm}^{-1}\right]$ & 1.958 & 2.008 & 2.287 & 2.429 \\
\hline$F(000)$ & 864 & 2084 & 2112 & 1728 \\
\hline$\Theta$ range $\left[{ }^{\circ}\right]$ & 1.486 to 27.483 & 1.786 to 29.130 & 2.055 to 29.117 & 1.893 to 27.200 \\
\hline total reflns & 56923 & 195002 & 65376 & 87459 \\
\hline unique reflns & 11737 & 27049 & 12066 & 9184 \\
\hline Rint & 0.0478 & 0.0440 & 0.0432 & 0.0623 \\
\hline observed reflns $(\mid>2 \sigma)$ & 10141 & 23803 & 10379 & 8005 \\
\hline Data/restraints/parameter & $\begin{array}{l}11737 / 741 / \\
727\end{array}$ & 27049 / 29 / 1169 & 12066 / 55 / 632 & 9184 / 0 / 466 \\
\hline $\mathrm{R} 1 / \mathrm{wR} 2(\mathrm{I}>2 \sigma)^{[\mathrm{a}]}$ & $0.0290 / 0.0613$ & $0.0205 / 0.0470$ & $0.0279 / 0.0635$ & $0.0323 / 0.0610$ \\
\hline R1/wR2 (all data) ${ }^{[a]}$ & $0.0392 / 0.0643$ & $0.0260 / 0.0491$ & $0.0352 / 0.0667$ & $0.0402 / 0.0628$ \\
\hline $\mathrm{GOF}^{[a]}$ & 1.073 & 1.028 & 1.036 & 1.193 \\
\hline $\begin{array}{l}\text { largest diff. peak and } \\
\text { hole }\left[\mathrm{e} \AA^{-3}\right]\end{array}$ & $\begin{array}{l}1.120 \\
\text { and }-0.823\end{array}$ & 1.107 and -0.714 & 1.545 and -0.705 & $\begin{array}{l}1.459 \\
\text { and }-1.855\end{array}$ \\
\hline
\end{tabular}

[a] R1 $=\Sigma(|| \mathrm{F} 0|-| \mathrm{Fc}||) / \Sigma|\mathrm{F} 0|, \mathrm{F} 0>4 \mathrm{~s}(\mathrm{~F} 0) . \mathrm{wR} 2=\left\{\Sigma\left[\mathrm{w}(\mathrm{F02}-\mathrm{Fc} 2) 2 / \Sigma\left[\mathrm{w}(\mathrm{F} 02)^{2}\right]\right\}^{1 / 2}\right.$. 
Table S1 continued.

Comprehensive crystallographic data for compounds 5-Y, 5-Lu, 6-Y, and 6-Lu

\begin{tabular}{|c|c|c|c|c|}
\hline & $5-Y$ & 5-Lu & $6-Y$ & 6-Lu \\
\hline CCDC & 1952865 & 1952858 & 1952868 & 1952860 \\
\hline formula & $\mathrm{C}_{33} \mathrm{H}_{52} \mathrm{BN}_{6} \mathrm{OY}$ & $\mathrm{C}_{33} \mathrm{H}_{52} \mathrm{BLuN}_{6} \mathrm{O}$ & $\mathrm{C}_{44} \mathrm{H}_{68} \mathrm{BN}_{6} \mathrm{OY}$ & $\mathrm{C}_{44} \mathrm{H}_{68} \mathrm{BLuN}_{6} \mathrm{O}$ \\
\hline $\mathrm{M}\left[\mathrm{g} \mathrm{mol}^{-1}\right]$ & 648.52 & 734.58 & 796.76 & 882.82 \\
\hline crystal system & monoclinic & monoclinic & monoclinic & triclinic \\
\hline space group & $P 21 / n$ & $P 21 / n$ & $P 2{ }_{1} / n$ & $P \overline{1}$ \\
\hline$a[\AA]$ & $11.8874(2)$ & 11.8831(5) & $20.0903(12)$ & $10.6560(5)$ \\
\hline $\mathrm{b}[\AA]$ & $19.2010(3)$ & $19.2344(8)$ & $10.2628(6)$ & 12.6363(5) \\
\hline$c[\AA]$ & $15.5311(3)$ & $15.4889(7)$ & $22.1377(13)$ & $17.0849(7)$ \\
\hline$\alpha\left[^{\circ}\right]$ & 90 & 90 & 90 & $76.3380(10)$ \\
\hline$\beta\left[^{\circ}\right]$ & $96.7110(10)$ & $96.3300(10)$ & $104.0960(10)$ & $82.3050(10)$ \\
\hline $\mathrm{Y}\left[{ }^{\circ}\right]$ & 90 & 90 & 90 & $87.5710(10)$ \\
\hline $\mathrm{V}\left[\AA^{3}\right]$ & $3520.68(11)$ & $3518.6(3)$ & $4427.0(5)$ & $2215.16(16)$ \\
\hline Z & 4 & 4 & 4 & 2 \\
\hline $\mathrm{T}[\mathrm{K}]$ & $160(2)$ & $180(2)$ & $99(2)$ & $100(2)$ \\
\hline$\rho_{\text {calcd }}\left[\mathrm{g} \mathrm{cm}^{-3}\right]$ & 1.224 & 1.387 & 1.195 & 1.324 \\
\hline$\mu\left[\mathrm{mm}^{-1}\right]$ & 1.689 & 2.838 & 1.356 & 2.267 \\
\hline$F(000)$ & 1376 & 1504 & 1704 & 916 \\
\hline$\Theta$ range $\left[{ }^{\circ}\right]$ & 1.693 to 27.875 & 1.694 to 30.119 & 1.897 to 27.099 & 1.659 to 30.538 \\
\hline total reflns & 44128 & 62037 & 79010 & 99941 \\
\hline unique reflns & 8401 & 10339 & 9763 & 13480 \\
\hline Rint & 0.0371 & 0.0476 & 0.0777 & 0.0362 \\
\hline observed reflns $(\mid>2 \sigma)$ & 6562 & 8229 & 7940 & 12838 \\
\hline Data/restraints/parameter & $8401 / 0$ / 398 & $10339 / 0 / 398$ & $9763 / 333 / 584$ & $13480 / 0 / 500$ \\
\hline $\mathrm{R} 1 / \mathrm{wR} 2(\mathrm{I}>2 \sigma)^{[\mathrm{a}]}$ & $0.0365 / 0.0755$ & $0.0273 / 0.0581$ & $0.0333 / 0.0765$ & $0.0172 / 0.0407$ \\
\hline R1/wR2 (all data) ${ }^{[a]}$ & $0.0583 / 0.0821$ & $0.0406 / 0.0637$ & $0.0480 / 0.0826$ & $0.0186 / 0.0413$ \\
\hline $\mathrm{GOF}^{[\mathrm{a}]}$ & 1.017 & 1.025 & 1.006 & 1.055 \\
\hline $\begin{array}{l}\text { largest diff. peak and hole } \\
{\left[\mathrm{e} \AA^{-3}\right]}\end{array}$ & $\begin{array}{l}0.536 \\
\text { and }-0.704\end{array}$ & $\begin{array}{l}2.338 \\
\text { and }-0.919\end{array}$ & $\begin{array}{l}0.384 \\
\text { and }-0.335\end{array}$ & $\begin{array}{l}2.016 \\
\text { and }-0.752\end{array}$ \\
\hline
\end{tabular}

[a] R1 $=\Sigma(|| \mathrm{F} 0|-| \mathrm{Fc}||) / \Sigma|\mathrm{F} 0|, \mathrm{F} 0>4 \mathrm{~s}(\mathrm{~F} 0) . \mathrm{wR} 2=\left\{\Sigma\left[\mathrm{w}(\mathrm{F} 02-\mathrm{Fc} 2) 2 / \Sigma\left[\mathrm{w}(\mathrm{F} 02)^{2}\right]\right\}^{1 / 2}\right.$. 
Table S1 continued.

Comprehensive crystallographic data for compounds 7-Y, 7-Lu, 8-Y, and 9-Lu

\begin{tabular}{|c|c|c|c|c|}
\hline & $7-Y$ & 7-Lu & $8-Y$ & 9-Lu \\
\hline CCDC & 1952863 & 1952857 & 1952867 & 1952864 \\
\hline Formula & $\mathrm{C}_{33} \mathrm{H}_{46} \mathrm{BF}_{6} \mathrm{~N}_{6} \mathrm{OY}$ & $\mathrm{C}_{33} \mathrm{H}_{46} \mathrm{BF}_{6} \mathrm{LuN}_{6} \mathrm{O}$ & $\mathrm{C}_{57} \mathrm{H}_{95} \mathrm{AlBN}_{10} \mathrm{OY}$ & $\mathrm{C}_{39} \mathrm{H}_{62} \mathrm{BLuN}_{6} \mathrm{O}$ \\
\hline $\mathrm{M}\left[\mathrm{g} \mathrm{mol}^{-1}\right]$ & 756.48 & 842.54 & 1063.12 & 816.72 \\
\hline Crystal system & monoclinic & monoclinic & triclinic & monoclinic \\
\hline Space group & $\mathrm{P} 21$ & $\mathrm{P} 21 / \mathrm{c}$ & $\mathrm{P} \overline{1}$ & $\mathrm{P} \mathrm{21/c}$ \\
\hline$a[\AA]$ & $20.8816(15)$ & $20.8123(15)$ & $16.8784(16)$ & $17.0580(12)$ \\
\hline $\mathrm{b}[\AA]$ & $21.1727(15)$ & $21.1757(15)$ & $20.723(2)$ & $11.9100(9)$ \\
\hline$c[\AA]$ & $18.8326(13)$ & $18.8646(14)$ & $20.752(2)$ & $20.5209(15)$ \\
\hline$\alpha\left[^{\circ}\right]$ & 90 & 90 & $61.930(2)$ & 90 \\
\hline$\beta\left[^{\circ}\right]$ & $116.7770(10)$ & $116.879(2)$ & $72.578(3)$ & $110.9950(10)$ \\
\hline $\mathrm{Y}\left[{ }^{\circ}\right]$ & 90 & 90 & $79.844(3)$ & 90 \\
\hline $\mathrm{V}\left[\AA^{3}\right]$ & $7433.4(9)$ & \begin{tabular}{|l}
$7415.7(9)$ \\
\end{tabular} & $6105.6(10)$ & $3892.3(5)$ \\
\hline Z & 2 & 8 & 4 & 4 \\
\hline $\mathrm{T}[\mathrm{K}]$ & $100(2)$ & $100(2)$ & $100(2)$ & $100(2)$ \\
\hline$\rho_{\text {calcd }}\left[\mathrm{g} \mathrm{cm}^{-3}\right]$ & 1.352 & 1.509 & 1.157 & 1.394 \\
\hline$\mu\left[\mathrm{mm}^{-1}\right]$ & 1.633 & 2.727 & 1.014 & 2.574 \\
\hline$F(000)$ & 3136 & 3392 & 2288 & 1688 \\
\hline$\Theta$ range $\left[{ }^{\circ}\right]$ & 1.211 to 28.282 & 1.097 to 30.032 & 1.477 to 30.184 & 1.338 to 27.483 \\
\hline total reflns & 148707 & 21614 & 265056 & 68987 \\
\hline unique reflns & 36898 & 21614 & 35933 & 8921 \\
\hline Rint & 0.0836 & 0.061 & 0.1098 & 0.0928 \\
\hline observed reflns $(\mid>2 \sigma)$ & 27179 & 18206 & 24681 & 6770 \\
\hline Data/restraints/parameter & $\begin{array}{l}36898 / 3181 / \\
1908\end{array}$ & 21614 / 69 / 919 & $35933 / 0 / 1341$ & $8921 / 36 / 485$ \\
\hline R1/wR2 (I>2б) ${ }^{[a]}$ & $0.0508 / 0.1086$ & $0.0386 / 0.0863$ & $0.0475 / 0.0946$ & $0.0284 / 0.0555$ \\
\hline R1/wR2 (all data) ${ }^{[a]}$ & $0.0834 / 0.1225$ & $0.0509 / 0.0923$ & $0.0900 / 0.1088$ & $0.0491 / 0.0617$ \\
\hline $\mathrm{GOF}^{[\mathrm{a}]}$ & 1.021 & 1.073 & 1.018 & 1.017 \\
\hline $\begin{array}{l}\text { largest diff. peak and hole } \\
{\left[\mathrm{e} \AA^{-3}\right]}\end{array}$ & $\begin{array}{l}1.776 \\
\text { and }-0.366\end{array}$ & $\begin{array}{l}5.899 \\
\text { and }-1.015\end{array}$ & 0.639 and -0.541 & $\begin{array}{l}0.558 \\
\text { and }-0.681\end{array}$ \\
\hline
\end{tabular}

[a] R1 $=\Sigma(|| F 0|-| F c||) / \Sigma|F 0|, F 0>4 s(F 0) . w R 2=\left\{\Sigma\left[w(F 02-F c 2) 2 / \Sigma\left[w(F 02)^{2}\right]\right\}^{1 / 2}\right.$. 


\section{Cone angle calculations}

To calculate the mathematically exact cone angles a series of .xyz files was generated from the final .cif files with ORTEP. The Mathematica package was downloaded free of charge from http://www.ccqc.uga.edu/references/software.php.

The supplemental file [cone angle data.xyz] contains the computed Cartesian coordinates of all of the molecules reported in this study. The file may be opened as a text file to read the coordinates, or opened directly by a molecular modeling program such as Mercury (version 3.3 or later, http://www.ccdc.cam.ac.uk/pages/Home.aspx) for visualization and analysis.

The adopted van der Waals radii were $r=1.20,1.70,1.55,1.52,1.92 \AA$ for $\mathrm{H}, \mathrm{C}, \mathrm{N}, \mathrm{O}, \mathrm{B}$ in this order.

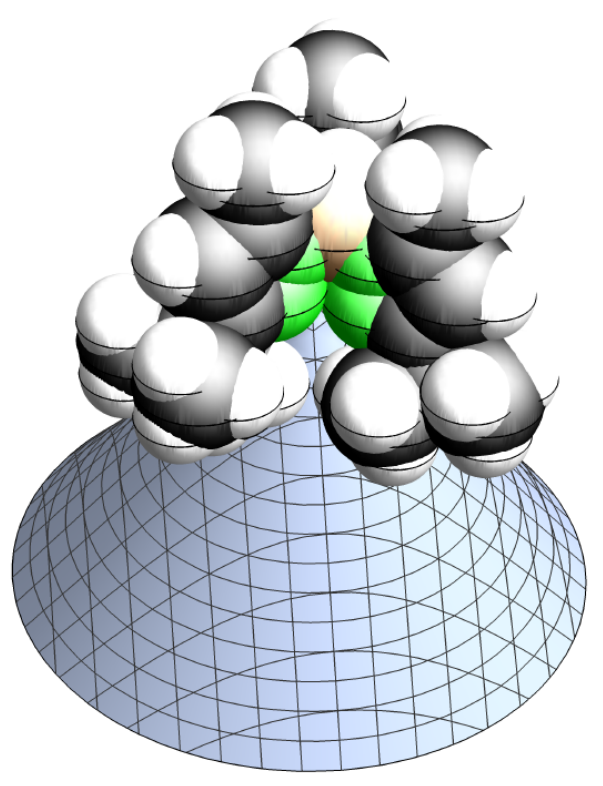

$\mathrm{Tp}^{\mathrm{tBu}, \mathrm{Me}} \mathrm{YMe}\left(\mathrm{AIMe}_{4}\right) \_\mathrm{a}$

Ligand atoms forming cone $=\{19,40,57\}$

Cone angle $(\mathrm{deg})=280.65$

Cone axis $=\{0.547789,0.180831,-0.81684\}$

$\mathrm{Tp}^{t \mathrm{Bu}, \mathrm{Me}} \mathrm{YMe}\left(\mathrm{AlMe}_{4}\right) \_\mathrm{b}($ cone depicted)

Ligand atoms forming cone $=\{29,45,68\}$

Cone angle $(\mathrm{deg})=276.12$

Cone axis $=\{0.446285,0.195798,-0.873208\}$

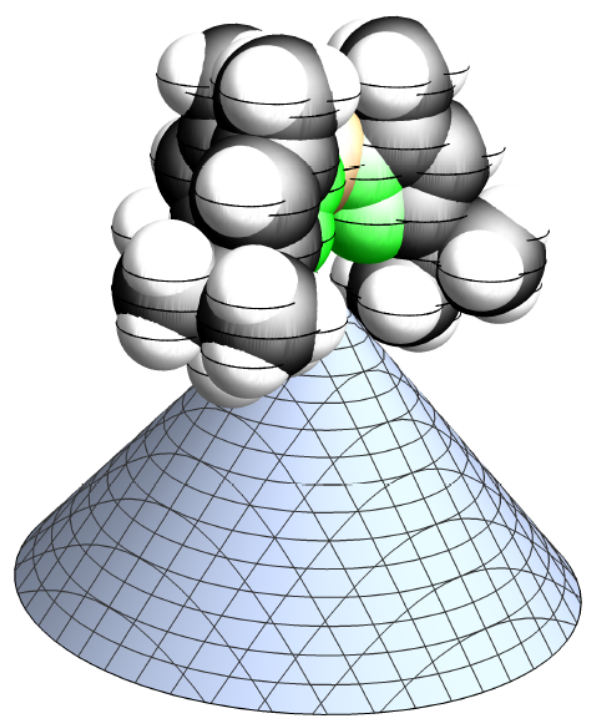

$\mathrm{Tp}^{\mathrm{tBu}, \mathrm{Me}} \mathrm{LuMe}_{2}$

Ligand atoms forming cone $=\{25,38,63\}$

Cone angle $(\mathrm{deg})=277.06$

Cone axis $=\{-0.894711,-6.37622 \times 10-17,-$ $0.446646\}$ 


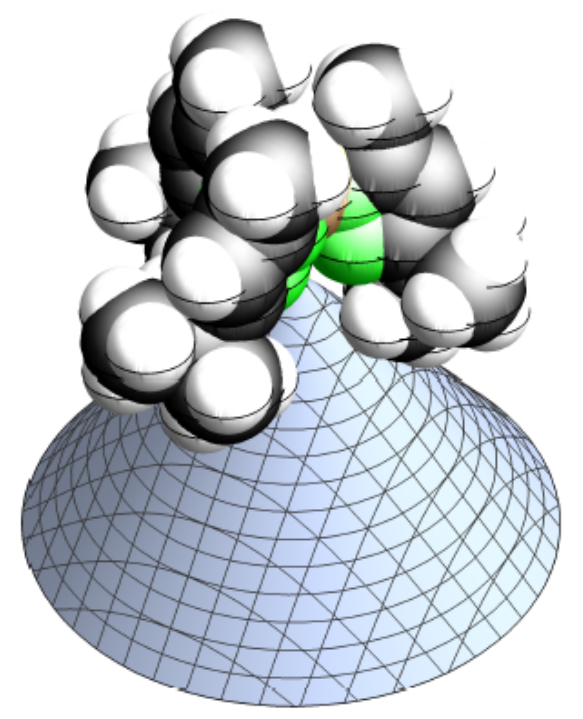

$$
\mathrm{Tp}^{\mathrm{tBu}, \mathrm{Me}} \mathrm{Lu}\left(\mathrm{OC}_{6} \mathrm{H}_{3} \mathrm{Me}_{2}-2,6\right)_{2}(1-\mathrm{Lu})
$$

Ligand atoms forming cone $=\{8,48,55\}$

Cone angle $(\mathrm{deg})=276.07$

Cone axis $=\{-0.225924,0.939593,0.257144\}$

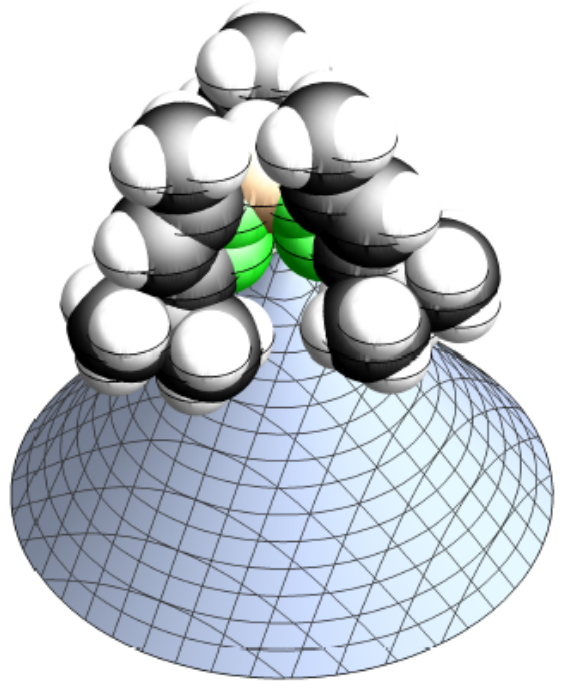

$\mathrm{Tp}^{\mathrm{tBu}, \mathrm{Me}} \mathrm{Lu}\left(\mathrm{OC}_{6} \mathrm{H}_{3} \mathrm{Pr}_{2}-2,6\right)_{2}$ (2-Lu)

Ligand atoms forming cone $=\{19,31,53\}$

Cone angle $(\mathrm{deg})=272.526$

Cone axis $=\{-0.166388,0.92943,0.329356\}$

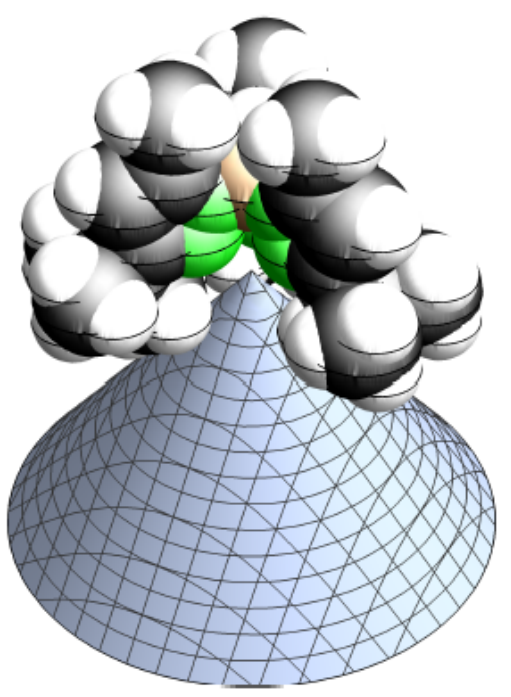

$\mathrm{Tp}^{t \mathrm{Bu}, \mathrm{Me}} \mathrm{Lu}\left[\mathrm{OC}_{6} \mathrm{H}_{3}\left(\mathrm{CF}_{3}\right)_{2}-3,5\right]_{2}(\mathbf{3 - L u})$

Ligand atoms forming cone $=\{20,26,54\}$

Cone angle $(\mathrm{deg})=280.424$

Cone axis $=\{-0.71205,-0.687129,0.144353\}$ 


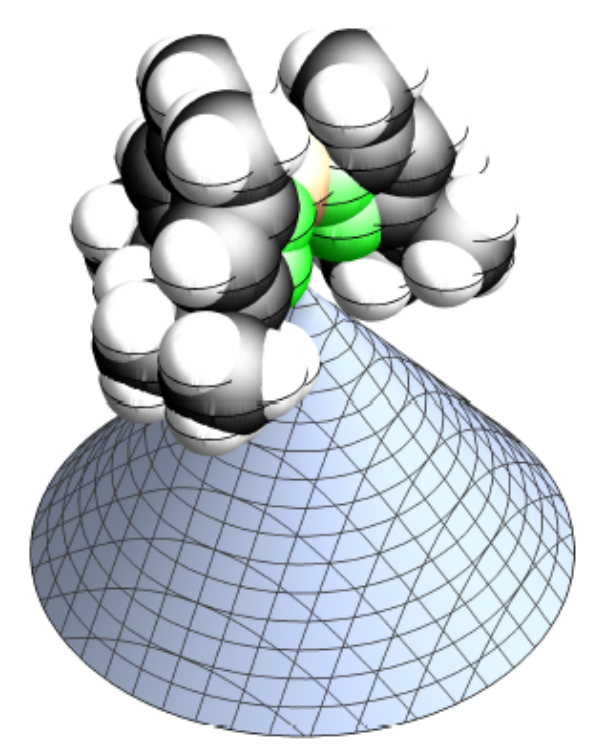

$\mathrm{Tp}^{t \mathrm{Bu}, \mathrm{Me}} \mathrm{LuMe}\left(\mathrm{OC}_{6} \mathrm{H}_{2} \mathrm{CBu}_{2}-2,6-\mathrm{Me}-4\right)(4-\mathrm{Lu})$
Ligand atoms forming cone $=\{21,43,44\}$

Cone angle $(\mathrm{deg})=278.118$

Cone axis $=\{-0.165494,0.984032,-0.0655229\}$

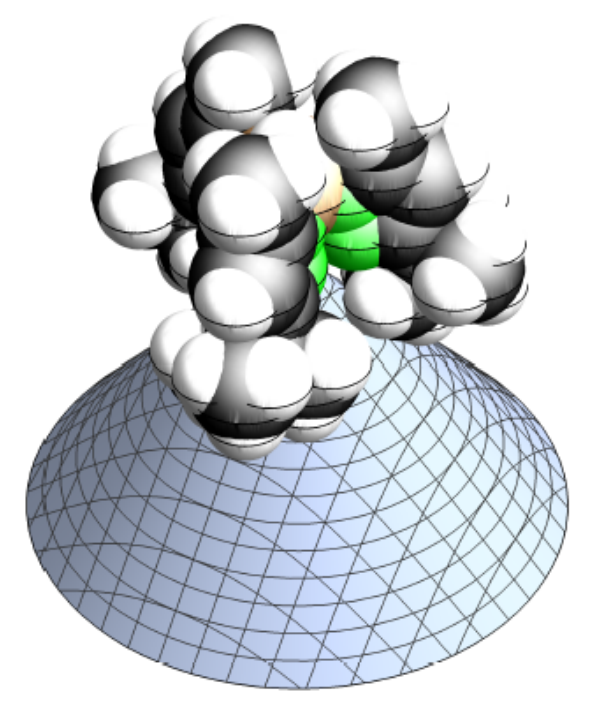

$\mathrm{Tp}^{\mathrm{tBu}, \mathrm{Me}} \mathrm{YMe}\left(\mathrm{OC}_{6} \mathrm{H}_{3} \mathrm{Me}_{2}-2,6\right)(\mathbf{5}-\mathrm{Y})$

Ligand atoms forming cone $=\{23,39,57\}$

Cone angle $(\mathrm{deg})=273.902$

Cone axis $=\{0.696277,-0.00548779,-0.717752\}$

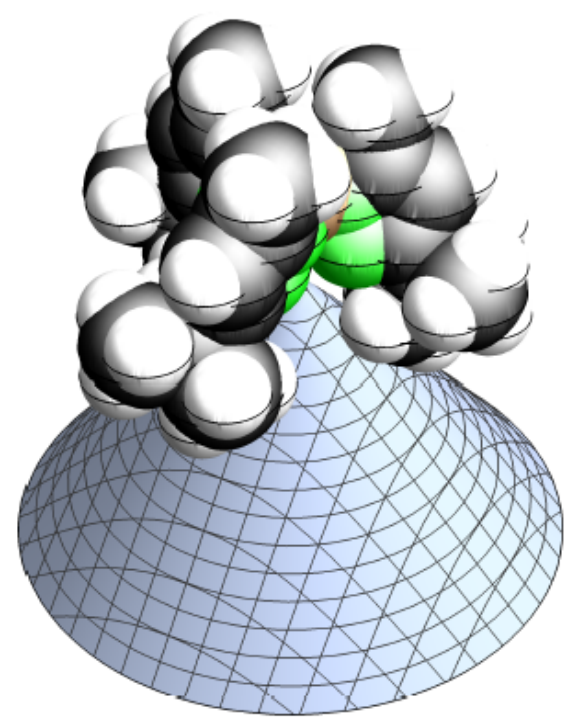

$\mathrm{Tp}^{t \mathrm{Bu}, \mathrm{Me}} \mathrm{LuMe}\left(\mathrm{OC}_{6} \mathrm{H}_{3} \mathrm{Me}_{2}-2,6\right)(\mathbf{5}-\mathrm{Lu})$

Ligand atoms forming cone $=\{8,48,55\}$

Cone angle $(\mathrm{deg})=276.07$

Cone axis $=\{-0.225924,0.939593,0.257144\}$ 


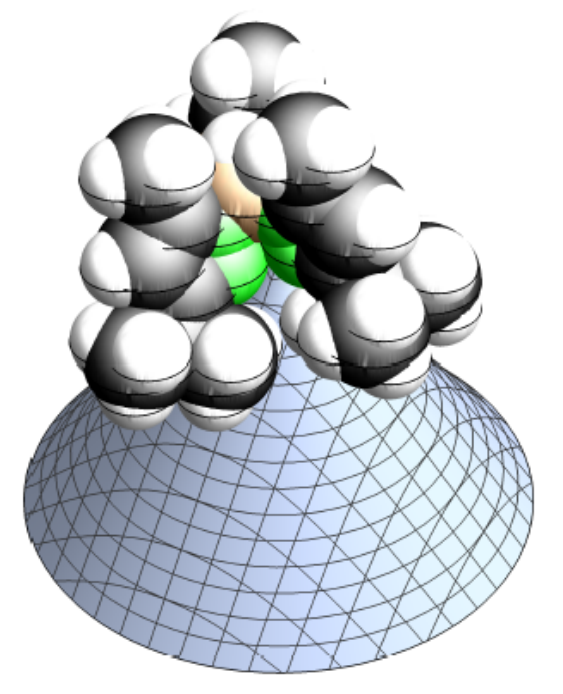

$\mathrm{Tp}^{t \mathrm{Bu}, \mathrm{Me}} \mathrm{YMe}\left(\mathrm{OC}_{6} \mathrm{H}_{3} i \mathrm{Pr}_{2}-2,6\right)(\mathbf{6 - Y})$

Ligand atoms forming cone $=\{13,46,67\}$

Cone angle $(\mathrm{deg})=276.057$

Cone axis $=\{-0.76823,-0.250027,-0.589329\}$

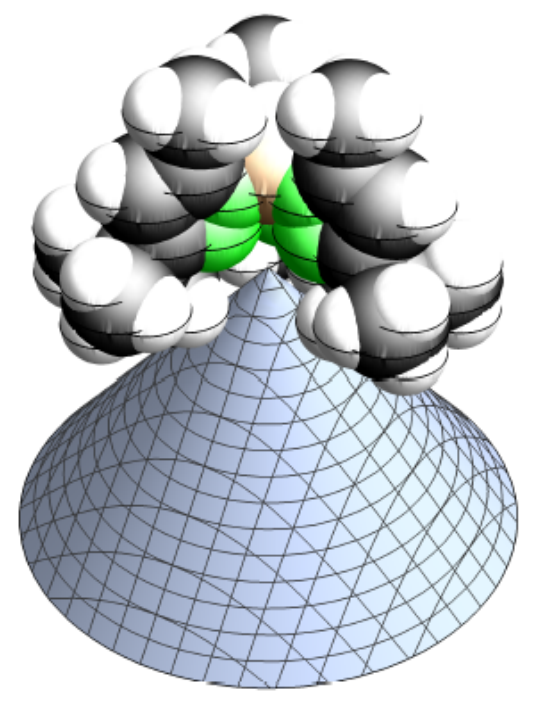

$\mathrm{Tp}^{\mathrm{ABu}, \mathrm{Me}} \mathrm{LuMe}\left(\mathrm{OC}_{6} \mathrm{H}_{3} / \mathrm{Pr}_{2}-2,6\right)($ 6-Lu)

Ligand atoms forming cone $=\{23,35,60\}$

Cone angle $(\mathrm{deg})=279.236$

Cone axis $=\{-0.999597,-0.0067429,-0.0275751\}$

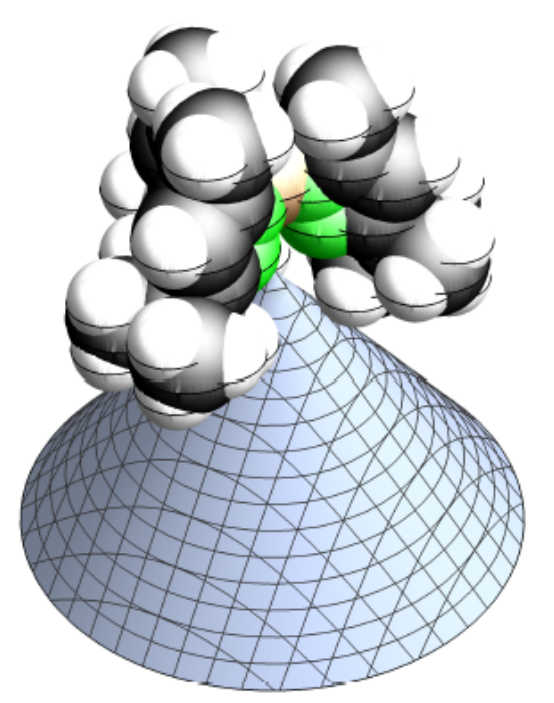

$\mathrm{Tp}^{\mathrm{tBu}, \mathrm{Me}} Y \mathrm{Me}\left[\mathrm{OC}_{6} \mathrm{H}_{3}\left(\mathrm{CF}_{3}\right)_{2}-3,5\right](7-\mathrm{Y})$

Ligand atoms forming cone $=\{23,34,58\}$

Cone angle $(\mathrm{deg})=278.637$

Cone axis $=\{-0.205908,0.616546,-0.759917\}$ 


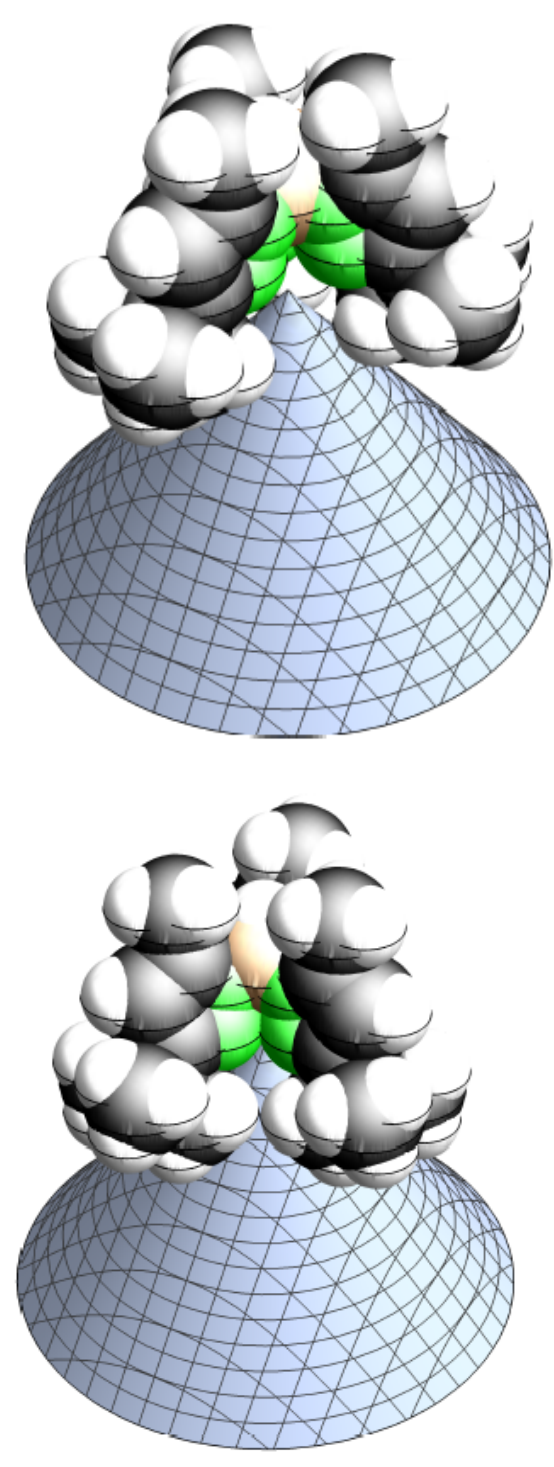

$\mathrm{Tp}^{\mathrm{tBu}, \mathrm{Me}} \mathrm{LuMe}\left[\mathrm{OC}_{6} \mathrm{H}_{3}\left(\mathrm{CF}_{3}\right)_{2}-3,5\right](7-\mathrm{Lu})$

Ligand atoms forming cone $=\{26,46,65\}$

Cone angle $(\mathrm{deg})=280.343$

Cone axis $=\{-0.627792,-0.76852,0.123506\}$

$\left[\mathrm{Tp}^{\mathrm{tB} u, \mathrm{Me}} \mathrm{YMe}(\mathrm{dmap})_{2}\right]\left[\mathrm{Me}_{3} \mathrm{AlOC}_{6} \mathrm{H}_{2} t \mathrm{Bu}_{2}-2,6-\mathrm{Me}-4\right]$ (8-Y)

Ligand atoms forming cone $=\{20,40,58\}$

Cone angle $(\mathrm{deg})=276.798$

Cone axis $=\{0.185673,-0.952331,-0.242058\}$

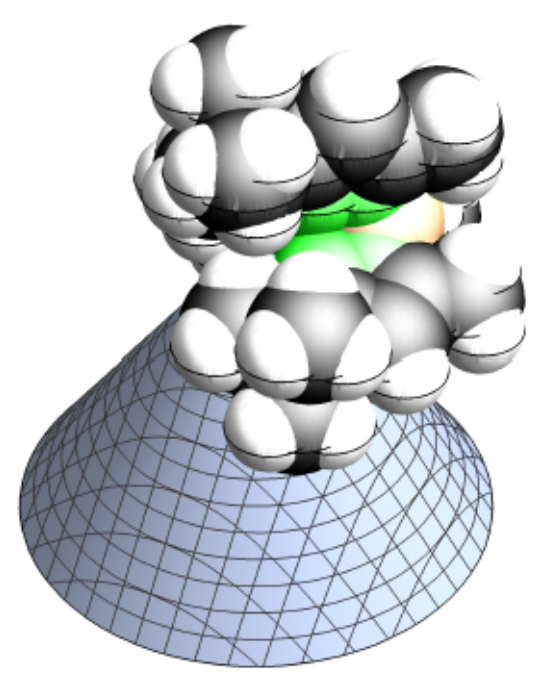

$\mathrm{Tp}^{\mathrm{tBu}, \mathrm{Me}} \mathrm{Lu}(\mathrm{BHT})_{\text {activated }}(\mathbf{9}-\mathbf{L u})$

Ligand atoms forming cone $=\{22,43,66\}$

Cone angle $(\mathrm{deg})=286.297$

Cone axis $=\{0.992352,-0.119976,0.0290265\}$ 